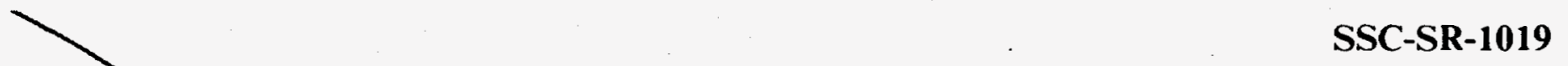

\title{
Preliminary Report on the Design of
}

The Superconducting Super Collider

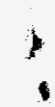

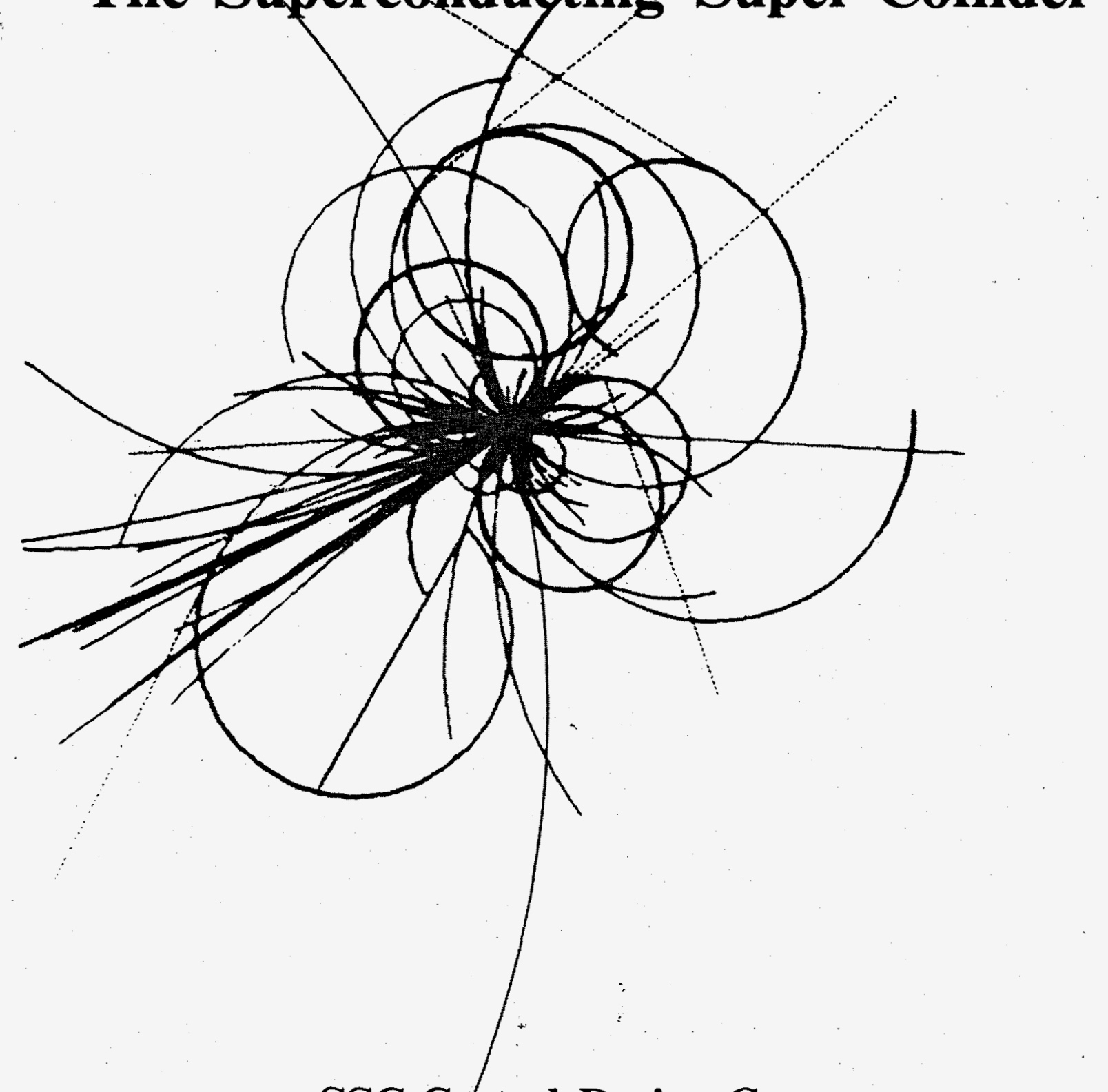

SSC Central Design Group

January 1986

APPROVED FOR RELEASE OR

PUBLICATION - O.R. PATENT T. IS S.

BY... 


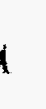

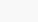




\section{DISCLAIMER}

Portions of this document may be illegible in electronic image products. Images are produced from the best available original document. 
PRELIMINARY REPORT ON THE DESIGN

OF THE SUPERCONDUCTING SUPER COLLIDER

\author{
Prepared \\ by \\ SSC Central Design Group \\ Universities Research Association \\ c/o Lawrence Berkeley Laboratory \\ Berkeley, California 94720
}

\title{
DISCLAIMER
}

This report was prepared as an account of work sponsored by an agency of the United States Government. Neither the United States Government nor any agency thereof, nor any of their employees, makes any warranty, express or implied, or assumes any legal liability or responsibility for the accuracy, completeness, or usefulness of any information, apparatus, product, or process disclosed, or represents that its use would not infringe privately owned rights. Reference herein to any specific commercial product, process, or service by trade name, trademark, manufacturer, or otherwise does not necessarily constitute or imply its endorsement, recommendation, or favoring by the United States Government or any agency thereof. The views and opinions of authors expressed herein do not necessarily state or reflect those of the United States Government or any agency thereof.

This work is supported by the U.S. Department of Energy under Contract No. DE-ACO2-76CH03000, ACcount 3001.

DISTRIBUTION OF THIS DOCUMENT IS UNI

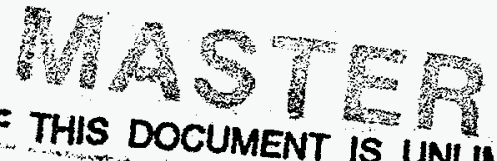


Preface

The Superconducting Super Collider (SSC) research and development program had its origins in a July 1983 recommendation of the High Energy Physics Advisory Panel, a nationally representative group of experts which advises the U.S. Department of Energy and the National Science Foundation. That recommendation urged prompt initiation of steps to assure the availability of a multi-TeV proton-proton collider of high luminosity for physics experiments at the earliest possible date. The Department of Energy responded positively and took a number of actions in FY 1984 to commence the necessary R\&D program. Among these was the commissioning of a SSC Reference Designs Study (RDS) in the first few months of 1984. The intense study examined the existing technology, delineated the important R\&D items, and made preliminary cost estimates of three SSC machines, based on three different proposed magnet styles. Largely on the basis of the RDS, the Department of Energy contracted with the Universities Research Association (URA), a consortium of 56 leading research universities, to conduct SSC R\&D, with the goal of firmly establishing the technical basis of the instrument and making a specific design with a detailed cost estimate. URA established the SSC Central Design Group (CDG) to coordinate the national R\&D effort. Work at the CDG began officially on 1 October 1984.

It has now been two years since the SSC Reference Designs Study. Much of the research and development indicated in that study has been carried out and the key design assumptions verified. A single magnet design has emerged from a rigorous selection process and much progress has been made in developing a facility design based on that superconducting magnet style. Many of the accomplishments of the R\&D program are documented in the SSC Interim Report (SSC-SR-1011, June 1985) and in the URA book let, "Supercollider R\&D: The First Two Years" (December 1985).

While a rather detailed Conceptual Design Report will be available in April, it is appropriate to give a preview, now that the primary parameters for the Conceptual Design Report have been put down. In this preview the leading two chapters give the historical and scientific-technical background for the SSC and deal at some length with the physics issues to be explored by the SSC. A third chapter reviews briefly the engineering and accelerator physics foundations for the developing SSC design, while the fourth lists the primary design parameters and describes the overall design. The fifth chapter describes briefly the principal engineering systems that will appear in the Conceptual Design Report, including the rather extensive injector system required. A sixth and final chapter outlines the beginnings of a "construction plan" put together for the purposes of exploring practical schedules and defining the critical design, development and planning paths for the overall facility and its major sub-systems.

The intent is to convey first of all the physics arguments that lead to the conviction that the SSC is a necessary next step for high energy physics research, and secondly the evidence that the technical basis for its creation as a cost-effective and reliable scientific instrument is at hand. The specifics of the proposed realization and a plan and schedule for construction are outlined in broad terms in order to set the stage for the conceptual Report to follow. 
This work is being carried out as a cooperative national effort of physicists and engineers, within the CDG, at the national laboratories, the universities, other research centers and in industry. Colleagues from many other countries, seconded from sister institutions abroad, provide key help through participation in technical task forces and at the CDG.

M. Tigner

January 20, 1986 
Table of Contents

Page

Chapter 1 Introduction to Particle Physics and Accelerators

1.1 Introduction

1.2 History of Particle Physics

1.3 Standard Model

1.4 Outstanding Problems

1.5 Existing and Proposed Collider-Accelerators

1.6 Synchrotron Colliders

Chapter 2 Scientific Need for the SSC

2.1 Physics at SSC Energies

2.2 Concepts of Very High Energy Collisions

2.3 Discovery Potential of a Multi-TeV Collider 34

2.4 Recapitulation

Chapter 3 Technical Basis for the SSC

3.1 Introduction

3.2 Magnet Development

3.3 Superconductor Development

3.4 Beam Dynamics and Aperture

3.5 Synchrotron Radiation and Vacuum

3.6 Conclusion

4.1 Introduction

4.2 Principal Parameters of the SSC

4.3 Layout and Beam Optics of the SSC

4.4 Conventional Systems

Chapter 5 Technical Systems

5.1 Collider Magnet System

5.2 Cryogenic System

5.3 Other Collider Systems

$\mathbf{5 . 4}$ Injector Systems

$\begin{array}{lll}\text { Chapter } 6 & \text { Preliminary Construction Plan } & 103\end{array}$

6.1 Summary Construction Schedule 103

6.2 Magnet Fabrication $\quad 106$

6.3 Injector Systems 106

$\begin{array}{lll}6.4 & \text { Comments } & 106\end{array}$

$\begin{array}{lll}\text { Chapter } 7 & \text { Summary and Conclusions } & 109\end{array}$ 
Chapter 1

\section{INTRODUCTION TO PARTICLE PHYSICS AND ACCELERATORS}

\subsection{INTRODUCTION}

High energy physics is the name given today to the effort to identify and understand the most fundamental structure of the material world. It is the continuation of the work of those who, in the decades around the turn of the century, discovered the oneness of light, electricity and magnetism, and first discerned and explored the atom. As exploration has progressed to smaller and smaller reaches within the atom (indicated schematically in Fig. 1-1), larger and larger apparatuses have been required. These efforts, carried out primarily by physicists in the United States and Canada, Europe, Japan and the Soviet Union, have produced a remarkable picture of the microcosm. Within the past twenty years, a new sublevel of matter smaller than the proton has become known. Within the past two years there has been experimental confirmation that the phenomena of radioactivity and ordinary light are intimately related. These advances, and others, are not only striking in themselves but have far reaching consequences. They enable us to ask deeper questions than ever before. What is the origin of matter? How did the fundamental particles and forces work together to produce the world around us today?

The domain of high energy or particle physics seems far from our everyday experience. High energy accelerators and detectors are the light sources and microscopes used to look inside the proton and neutron which are themselves a hundred thousand times smaller than an atom. They produce and analyze collisions of fundamental particles producing in turn new particles traveling at velocities near the speed of light. In this domain times are measured in billionths of a second. As remote as these conditions seem to us, there was a time at the beginning of the universe when such conditions prevailed. The expanding universe was filled with the myriad exotic particles now investigated by high energy physicists. The interactions of these particles shaped the development of the universe to the state which we marvel at today.

While this explosion of new knowledge gleaned from the search for the fundamental over the past century profoundly deepened and broadened our world view, the practical consequences of this knowledge have also been universally felt. In addition to the intellectual and cultural wealth generated by these most basic scientific pursuits, enormous economic wealth has accrued to those societies able to make and understand these discoveries and turn the new knowledge to practical ends. The drive to elucidate the nature of electricity provided the foundations upon which scientists and engineers built the electrical industry. This, together with the discovery of quantum mechanics which flowed from the exploration of the atom, provided the foundations for our modern electronics industry and has illuminated the development of many other engineering sciences. The exploration of the atomic nucleus taught us to win energy from the transmutation of the elements through controlling the fission process and has brought us tantalizingly close to controlling the even more efficient fusion process. Mastering it may well require a yet deeper understanding of the fundamental material constituents and their interactions. 

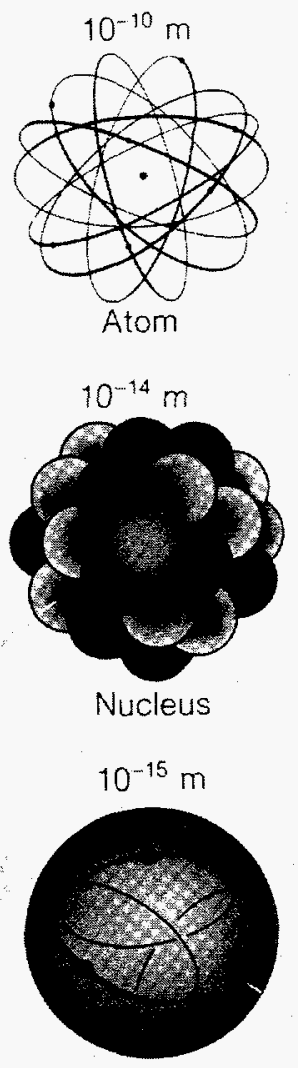

Proton
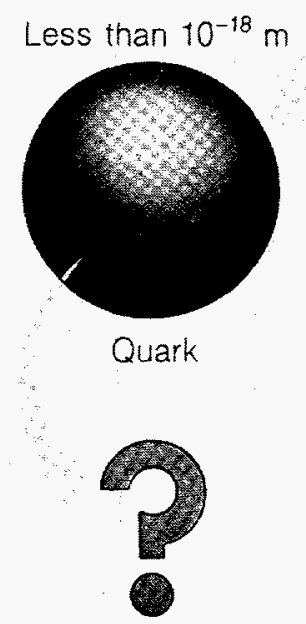

Figure 1-1. The progression of investigations at smaller and smaller scales requires accelerators of larger and larger size. The SSC will probe to distances of $10^{-19} \mathrm{~m}$ and less. 
If history is any guide it will be the unexpected discoveries still waiting to be made that will shock and inform our picture of the material world and richly reward those prepared to grasp their meaning and develop their consequences.

\subsection{HISTORY OF PARTICLE PHYSICS}

To understand the goals of particle physics, it is important to recollect its early history. The experimental achievements of J.J. Thomson, the Curies, Rutherford, and their contemporaries led to the picture of matter as composed of atoms, each having a heavy, small nucleus surrounded by a much larger cloud of light electrons. This work was the basis for the discovery in the 1920's of quantum mechanics which provides the foundation for our understanding of the behavior of all matter.

The modern picture of the atom was first developed by Niels Bohr in 1913, but was not really completed until the discovery of the neutron in 1932 . The protons and neutrons are bound together in the nucleus by the "strong" force. Particles experiencing the strong force are called hadrons. Those, like the electron, that do not experience this force, are called leptons. The negative electrons are bound to the positive nucleus by the electromagnetic force. This can be viewed as resulting from the emission and absorption of light in discrete quanta called photons. Although the constituents of everyday matter had been found, there remained unexplained phenomena. Radioactivity, first discovered in the 1890 's, could be described only partially. There was little understanding of the forces holding the nucleus together. Moreover, observations of cosmic rays soon produced startling results: the observation of new particles never encountered in ordinary matter - antiparticles, muons, pions, and kaons. Starting in about 1950, advances were made rapidly as the introduction of high energy particle accelerators superseded cosmic ray studies. It became possible to control the experimental conditions by providing beams of particles to a specified location and with a specified energy, rather than relying on random events occurring beyond experimental control. The high energy beams made possible the creation of new particles according to Einstein's relation, $E=m c^{2}$.

Tremendous technical advances contributed to the experimental progress. Measurements had to be made of particles moving nearly at the speed of light, living only fractions of a billionth of a second. Enormous quantities of data strained the capacities of existing computers. The recording of collisions of high energy particles required new techniques in optics and electronics. These demands were met with increasingly advanced instrumentation.

Soon patterns began to emerge in the data. The new particles could be grouped into a table like the periodic table of elements. Each particle could be characterized by its mass and its electric charge. Other characteristics emerged. Particles came in sets - a pair like the proton and neutron, a triplet of pions of different electric charges, $\pi^{+}, \pi^{0}, \pi^{-}$. Each particle in a set had nearly the same mass. Another characteristic was identified and dubbed "strangeness." Strangeness is akin to electric charge. Electric charges are made in pairs: an atom can lose an electron (charge -1) and become a positive ion (charge +1 ). Strange particles were produced in pairs, one 
with positive strangeness, one with negative strangeness. Unlike electric charge, strangeness could be lost altogether through relatively slow processes called weak interactions. Thus a $K^{+}$particle (strangeness +1 ) can turn into a $\pi^{+}$and $\pi^{0}$, neither of which has strangeness.

Further discoveries disclosed that larger groupings could be made, joining several sets of particles together. These theoretical structures were soon confirmed by experiments revealing predicted, but hitherto unseen, particles. These larger groupings organized the enormous number of new particles into a striking order. What was the reason for this ordering?

It was proposed in 1963 that all these subnuclear particles could be understood as being composed of still smaller entities - quarks. A hundred or more particles could suddenly be understood in simple terms. There were then three quarks imagined: $u, d$, and $s$. Three of these put together made a "baryon", a particle like the neutron ( 2 d's and a $u$ ) or proton ( 2 's and a d). One quark and one antiquark could combine to make a meson - the pion, kaon etc. Simple counting could predict new particles - and they were found.

At first, the quarks seemed like artificial constructs. No quarks had ever been seen, so many thought they were simply a convenient mathematical fiction. Such was the attitude at first, but a series of important experiments over the next ten years provided convincing evidence that the quarks were real.

The construction of the Stanford Linear Accelerator Center (SLAC) provided an extremely powerful tool for exploration inside the proton. The accelerator produced electrons with unprecedented energy, nearly 20 billion electron volts $(20 \mathrm{GeV})$. When the electrons collided with protons, characteristics of these collisions gave evidence for the internal construction of the proton, just as Rutherford's alpha particles had sixty years earlier for the atom. Moreover, just as Rutherford had been surprised to find a hard nucleus inside a soft atom, the SLAC results showed that there were hard constituents - dubbed partons - inside the proton.

Similar experiments were done at the Fermi National Accelerator Laboratory (Fermilab) in Illinois and at CERN, the European facility in Geneva, Switzerland. Some of the experiments were done using neutrinos in place of electrons. Neutrinos are massless particles that are produced in radioactive decay and in the decay of particles like muons and pions. They interact only feebly with matter. Since they have no strong interactions, they are classed as leptons. Indeed, they can pass right through the earth undeflected. Occasionally a neutrino does collide with a proton or neutron, and those rare collisions can be studied. Once again, these collisions revealed point-like constituents within the proton. Moreover, when these collisions were compared with electron collisions at SLAC, the ratio of the two was just what would be expected if the hard objects inside the proton were quarks.

The reality of quarks was established convincingly with the simultaneous discovery in 1974 at the Brookhaven National Laboratory and at SLAC of an astonishing particle: $\mathrm{J} / \psi$. A series of remarkable results from SLAC and the West German laboratory, DESY, established that the $J / \psi$ was the first of a series of atom-like states, composed of a new quark, the c or charmed quark, 
and its antiquark. Similar states of a subsequent, fifth quark, denoted b, were discovered at Fermilab in 1977. Its properties have been extensively studied at Corne 11 and at DESY. Preliminary evidence for an expected sixth quark has been reported at CERN, but the data are not yet conclusive.

The most powerful forces we know of are those that bind the quarks together to form protons and neutrons. These are termed "strong" forces. The other forces are gravity, electromagnetism, and weak forces. The weak forces are responsible for beta-decay ( a form of radioactivity) and the interactions of neutrinos. In 1934, Enrico Fermi wrote down a theory that, with only minor modifications, still describes weak interactions at low energies. A major achievement occurred in 1956 when T.D. Lee and C. N. Yang noted that the weak interactions might violate parity, that is, they might distinguish between left and right in a fundamental way. It had previously been thought that if an experiment were done and at the same time was viewed both directly and in a mirror, both views would appear to be physically sensible. Lee and Yang proposed that this might not be so for the weak interaction and they were proved correct in experiments conducted soon after. The results were incorporated in an improved version of the Fermi theory adequate to describe the experiments that had been done at Fermilab and CERN exploring the structure of the proton using neutrino beams.

In 1973, a group working with a bubble chamber at CERN announced a startling result. Previously, all scattering events with a neutrino incident, resulted in a charged electron or muon going out. Such events were called charged current events since charge had to be transferred to the neutrino, a neutral lepton, to make it into a charged lepton. The CERN group had convincing evidence of events in which the neutrino emerged, still uncharged. These were called neutral current events. They were analogous to the electron scattering at SLAC where the incident electron interacted electromagnetically (via photon exchange) with the proton, but still emerged as an electron. Since the electron was viewed there as emitting a neutral particle--the photon--the neutrino in the CERN experiment had to be viewed as emitting a neutral particle. This neutral particle could not be the photon, since the photon sees only charged particles and the neutrino is chargeless. There had to be another neutral particle in addition to the photon.

In the Fermi theory, charged particles, the $W^{+}$and $W^{-}$, could account for the weak force the way the photon accounted for the electromagnetic force. While the photon was massless, the $W$ would be quite massive. Its great mass would account for the feebleness of the weak force. The discovery of neutral currents at CERN, subsequently confirmed at Fermilab and Argonne National Laboratory, indicated that an additional heavy particle was called for. It was denoted by $Z$. Such a particle had been hypothesized independently by Glashow, Weinberg, and Salam some years earlier, and work in the early $70^{\prime}$ 's showed that the theories containing the $Z$ were renormalizable - a technical word which might be translated as "calculable."

The theories containing the $W$ and the $Z$ were all the more remarkable because they united the weak and electromagnetic interactions, an accomplishment reminiscent of the unification of electricity and magnetism by James Clerk Maxwell in the middle of the last century. They contained four force 
carrying particles: $W^{+}, W^{-}, Z$, and the photon. These fitted together in an essential way. Neutral currents provided strong circumstantial evidence for the theory. Stronger evidence came from an experiment at SLAC on the scattering of polarized electrons which showed parity violation, the signature of exchange of both a photon and a $Z$ with the target proton. The unified electroweak theory looked convincing. A key prediction stood out. The extensive neutral current experiments performed in the 1970's made it possible to predict the masses of the $W$ and $Z$.

The predicted masses were between 80 and $100 \mathrm{GeV}$, too heavy to be produced at existing machines. CERN responded by developing an innovative way to use its $400 \mathrm{GeV}$ machine (similar to the one at Fermilab at the time), which could normally produce particles with a mass no more than about $10 \mathrm{GeV}$. The inefficiency is due to a simple fact: conservation of momentum. When a $400 \mathrm{GeV}$ proton strikes a stationary target, the resulting products must have $400 \mathrm{GeV}$ of momentum. Thus much of the initial energy of the collision goes into the kinetic energy of the products, rather than into making new mass. CERN had previously built the Intersecting Storage Rings (ISR) to collide protons with protons at a beam energy of $30 \mathrm{GeV}$. That required two separate rings, with protons circulating clockwise in one and counter-clockwise in the other. To exploit the existing much larger $400 \mathrm{GeV}$ accelerator (SPS) without building another ring, CERN decided to send antiprotons around the SPS same ring, going the opposite way. This had already been done for electrons and positrons, but that was enormously simpler. The problem with antiprotons was to make enough of them, and confine them into a small beam.

In 1983, CERN succeeded in this enterprise. It was thus able to reach a useful energy of $540 \mathrm{GeV}$, whereas the SPS alone or Fermilab machine using a stationary target could reach only about $30 \mathrm{GeV}$. The project paid off handsomely. Both the $W$ and the $Z$ were discovered at the predicted masses.

The investigation of the $W$ and $Z$ has just begun. SLAC is pursuing an ingenious plan to study the $Z$, using a modification of its existing accelerator. Meanwhile, CERN is constructing a new accelerator, $27 \mathrm{kilometers}$ in circumference, to study first the $Z$ and then the $W$. Fermilab has completed and tested a large collider similar to CERN's, but at several times the energy. The results of these new accelerators are sure to enrich our understanding of these new fundamental particles.

What lies beyond the $W$ and $Z$ ? To reach much further, we need a machine with capabilities far greater than those now nearing completion. The SSC is designed to be that machine.

\subsection{THE STANDARD MODEL}

What are the basic building blocks of matter? The answer to this question has changed as we have learned more and more. In the 19th century, chemists established the existence of atoms as the fundamental units of matter. As mentioned earlier, the inner structure of the atom was determined starting in the 1890's and culminating in the early 1930's. The atom is composed of electrons surrounding a much tinier and more massive nucleus, which is itself composed of protons and neutrons. 
So far as has been determined, the electron still appears elementary, that is, it does not seem to have an inner structure. As discussed in the previous section, the situation is different for the proton and neutron. They are composed of quarks - two u-quarks and one d-quark for a proton and two d-quarks and one u-quark for a neutron. A neutron, because it is a bit more massive than a proton, is not stable but instead decays to a proton, an electron and an electrically neutral particle called a neutrino (actually, an antineutrino). This decay can be viewed as the change of a d-quark to a u-quark, an electron, and an antineutrino through the action of the weak force.

The four particles, the u-quark, the d-quark, the electron, and the neutrino form a set we refer to as a "generation". All but the neutrino have electric charge and thus participate in electromagnetic interactions. The two quarks experience the "strong force" that binds quarks together to form protons and neutrons. This force is analogous to the electromagnetic force except that it depends not on electric charge, but on the so-called "colorcharge". The electron and neutrino do not experience the strong force; they have no color-charge.

The first generation - the u-quark, the d-quark, the electron, and the neutrino - is enough to make all the ordinary matter of atoms. However, two other generations have been discovered. This is displayed in Fig. 1-2. The second generation consists of the c-quark (charmed-quark), the s-quark (strange-quark), the muon, and another neutrino (called the muon-neutrino). Why there should be more than one generation is a puzzle. The third generation consists of the b-quark (bottom-quark), the yet-to-be-confirmed $t$-quark (top-quark), the tau, and a third neutrino (tau-neutrino).

Each generation parallels the first. All the quarks have color and participate in the strong interactions. The particles like the electron and neutrino that do not have color are called leptons. All but the neutrinos have electric charge and experience electromagnetic forces. The weak interactions can change $s$-quarks to $c$-quarks, and vice versa, just as they change $d$-quarks into u-quarks.

The standard model stipulates that there are two basic categories of particles: the matter particles which comprise the three generations of quarks and leptons, and the force particles (technically called "gauge" particles).

Figure 1-2 displays the force particles. We have already cited examples in which the electromagnetic force is transmitted from one charged particle to another by a quantum of electromagnetic energy--a photon. Similarly, the strong force is transmitted from one colored particle to another by a quantum called the gluon (since it "glues" the quarks together to form the protons and neutrons). The gluon is more complex than the photon because, while the photon is itself neutral, the gluon carries color charge. Thus gluons can interact with each other in a way in which photons cannot. The consequences of this are dramatic. Two electrically charged objects can be pulled apart far enough that the force between them becomes negligible. The same is not true for a quark and an antiquark. No matter how far apart they are pulled, the 
Constituent Particles in the Standard Model

\section{Force Particles}
Photon
W
Z
Gluon

\section{Matter Particles}

\begin{tabular}{l|l|l|l|l}
\multicolumn{1}{c}{} & \multicolumn{2}{c}{ Quarks } & Leptons \\
1st generation & $\mathrm{u}$ & Up & $v_{\mathbf{e}}$ & e-Neutrino \\
& $\mathrm{d}$ & Down & $\mathrm{e}$ & Electron
\end{tabular}

2nd generation

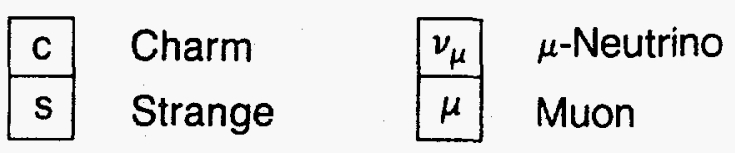

3rd generation
\begin{tabular}{|l|l}
\hline$t ?$ & Top \\
\hline$b$ & Bottom
\end{tabular}

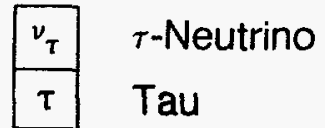

Figure 1-2. 
force between them does not decrease. For this reason, no quark has ever been seen in isolation.

The weak force is similarly transmitted by the exchange of the $\mathrm{W}^{+}, \mathrm{W}^{-}$, and $Z$ particles. We saw in the previous section that the weak and electromagnetic forces have a common origin, with the photon, and $W$ and the $Z$ being very similar particles in certain ways. For example, just as light (photons) can be polarized, so can the $W$ and $Z$. Unlike the photon, the $W$ and $Z$ are very massive particles, a difference that needs explanation.

The model shown in Fig. 1-2 is a remarkably successful one. There are no data that contradict the model at present. Nevertheless, it is almost certainly not the last word for there are many questions for which it provides no answer.

\subsection{OUTSTANDING PROBLEMS}

The photon seems most unlike the $W$ and $Z$. Photons surround us and are ultimately the source of our contact with the physical world. The $W$ and $Z$ were never observed directly before 1983. How can they be so analogous as to warrant being included in a single family, the force particles?

The interaction of light and matter was the first subject of investigation after the invention of quantum mechanics. P. A. M. Dirac found a way to incorporate special relativity and his theory was immediately successful in describing many physical phenomena. Unfortunately, his theory was only an approximation, albeit an excellent one. When the corrections to the theory were calculated, they turned out to be infinite! This puzzle was solved shortly after World War II, and the resulting theory, quantum electrodynamics (QED), has proved the most complete and successful physical theory ever developed.

Since QED works so well, it is natural to pattern a theory of the weak interactions after it. Difficulties arise because the weak force particles ( $W$ and $Z$ ) are massive--the success of QED seems to depend on the photon being massless. The conceptual way out of this predicament is to augment the simple theory, in which the $W$ and $Z$ are initially massless, by the addition of a "field" pervading all space uniformly and interacting with the $W$ and $Z$ to give them their masses. The theory turns out to be like QED in that its corrections can be calculated and are finite, while the $W$ and $Z$ acquire masses. In fact, all the particles, the quarks and leptons included, start out being massless and only obtain mass from this mysterious "field", which might be thought of as a ubiquitous molasses, slowing down all the particles, since without it, they would all be massless and move at the speed of light. The idea of the mass-generating field is central to the development of the unified theory of weak and electromagnetic interactions discussed earlier.

Is there any way to verify that this mass-generating field is really there? Actually, there are several ways that this field can arise and each has its characteristic signals. In all cases, there must be new particles or a new interaction, so far undiscovered, that are the vestige of the mechanism 
that gives masses to most particles. We cannot say precisely where these new particles or interactions will be found, but there are general, persuasive arguments that some evidence must lie within the range of the SSC.

The simplest model for the mass-generating process requires that there be one more particle besides the matter and force particles described above. This particle is called the Higgs particle. It is a remnant of the omnipresent "field" giving mass to all other particles. Paradoxically, its own mass is unknown to us although its other properties can be predicted.

In another model, there are many particles analogous to the Higgs particle, but they are not fundamental. Instead they are composites of new particles rather like the known quarks or leptons. A more comprehensive theory called supersymmetry incorporates the mass-generating mechanism in a framework of twice as many basic constituents as are now known, each force and matter particle having a symmetry partner (see Sect. 2.1).

The search for new particles that are linked to the ultimate source of mass is a primary goal of the SSC, as is discussed in more detail in Chapter 2.

The standard model is incomplete in other ways. There is no understanding of why there are three generations. There is no understanding of why the quarks and leptons have the masses they do. Even if these masses arise from interaction with the mass-generating field, the actual values of the masses seem to be arbitrary.

Our lack of understanding of the masses of the various particles is a very deep problem. Not only are we unable to predict the masses of the quarks and leptons exactly, we cannot even provide a crude estimate. The masses of the $W$ and $Z$ were predicted before their discovery by comparison with the Fermi coupling constant, $G_{F}$, which is measured in beta-decay. However, we have no understanding of this number. It sets the scale for the $W$ and $Z$ masses to be about $100 \mathrm{GeV}$. No one can explain why the number was not a million times larger or a million times smaller. Another unexplained energy scale is related to gravity. Gravitational interactions between elementary particles are important only at energies of about $10^{19} \mathrm{GeV}$. This stupendous energy, nearly a billion billion times higher than the energies associated with the $W$ and $z$, is called the Planck scale. There is a great disparity between the Planck scale and the masses of the $W$ and $Z$. Why this is so is a mystery, often called the "hierarchy" problem because there is an unexplained hierarchy of masses or energies.

Between the $100 \mathrm{GeV}$ energy scale explored by the CERN and Fermilab Colliders and the Planck scale is an enormous terra incognita. The SSC would be the first machine to begin to explore this region of which we are so ignorant.

It has been suggested that the unification of weak and electromagnetic interactions is only the beginning. Perhaps the strong interactions are in some way united with the electroweak. This is a very attractive possibility. There could be extra force particles that could turn quarks into leptons. In that case the proton itself could decay, albeit very slowly, for example 
permitting $p \rightarrow \pi^{0} e^{+}$. Unfortunately, despite a major experimental effort, no evidence has been found for such processes.

There are reasons for suspecting that the electroweak and strong interactions should be unified. A powerful one is that the proton and electron have electric charges which are precisely equal and opposite. If there are force particles that permit the proton to decay into a positron and a neutral pion, the proton and positron must have precisely the same electric charge, and the proton and the electron precisely opposite charges.

While the possibility of uniting the strong interactions with the electroweak interactions has a strong esthetic appeal, there is a serious lack of pertinent experimental information. New discoveries at the SSC might provide a clue to the validity of this exciting hypothesis. For example, still heavier leptons of other generations may begin to manifest some internal structure, rather than being merely heavier point-like electrons.

\subsection{EXISTING AND PROPOSED COLLIDER-ACCELERATORS}

The progress of the last thirty-five years in particle physics has been dependent on the development of a series of ever more powerful accelerators. These are of two sorts: proton accelerators and electron accelerators. The beam of particles produced by the accelerator may then be directed at a stationary target. Some of the energy carried by each electron or proton is transformed into new matter according to Einstein's relation $E=m c^{2}$. The incident particle carries a great deal of momentum which has to be shared among the produced final particles. As a result, only a small fraction of the energy is actually available for production of new particles. To circumvent this problem, a new type of accelerator called a collider was developed. In a collider, there are two beams circulating in opposite directions. The momentum in one of the beams is just opposite the momentum in the other. Since the sum of the momenta is zero, none needs to be carried away by the final state and all the energy is available for particle production.

The first proton-proton collider (1971) was the Intersecting Storage Rings (ISR) at CERN. It had two rings with particles circulating in opposite directions. The proton beams collided with each other at several locations where the rings crossed. With $30 \mathrm{GeV}$ beams, it was equivalent to a machine with a single beam of energy $2000 \mathrm{GeV}$ bombarding a stationary target, an energy still not achieved to this day by a single-beam facility.

The effectiveness of a collider of a given energy is judged by its luminosity, a measure of the number of collision partners each proton encounters as the beams collide. The collision rate is proportional to the luminosity. Each process studied has an effective area or "cross section." If two protons approach each other, the likelihood they will collide depends on how large the protons appear, that is, their total cross section. This was measured to be about $45 \times 10^{-27} \mathrm{~cm}^{2}$ at the ISR. The rate of proton-proton collisions is given by the product of the cross section and the luminosity. The ISR reached a peak luminosity of about $10^{32} \mathrm{~cm}^{-2} \mathrm{~s}^{-1}$ corresponding to a rate of protonproton collisions of about 4 million per second. Of course, only a very small 
fraction of these events will be of interest: only part of the total cross section needs to be studied. In fact, a process in which a large mass, $M$, is created has a cross section that varies roughly as $1 / M^{2}$. If $M$ is very large, the cross section is very small. For this reason, it is important for a highenergy machine like the SSC to have a high luminosity.

Colliding beam machines have also been made with electrons and positrons (anti-electrons). Two such machines are operating at the Stanford Linear Accelerator Center, SPEAR, with a beam energy of 1.5 to $4 \mathrm{GeV}$ and PEP, with a beam energy of about $15 \mathrm{GeV}$. Similar machines have operated at the West German laboratory in Hamburg. DESY. A very successful electron-positron colliding beam accelerator, CESR, is located at Cornell University. The existing and planned colliding beam machines are listed in Table $I$.

Hadron colliders and electron-positron colliders have important similarities, but also important differences. Both are effective ways of producing very high useful energies. Electron-positron colliders can be tuned to produce a precise collision energy. This makes them ideal for studying a particle like the $Z$ which can be produced when an electron and positron of the right energy annihilate. In contrast to electrons and positrons, the proton is not elementary, but contains quarks and gluons which share the energy of the proton. As a result, a collision of one quark (or gluon) from each of the colliding protons uses only a fraction of the total proton-proton energy. This suggests that electron-positron machines would give higher effective collision energies since the colliding particles are not composed of smaller pieces. However, the hadron-hadron machines more than make up for this disadvantage because it is far easier to increase the beam energy of hadron machines. The reason is that the very light-weight electrons (but not protons) in a circular machine radiate enormous amounts of energy which must then be pumped back into the beam. To avoid this loss of energy by radiation, the accelerator at SLAC was built not as a circular machine, but as a straight one. Electron-positron machines with energies much greater than those at LEP (whose circumference will be $27 \mathrm{~km}$ ) will have to be linear colliders. The Stanford Linear Collider, SLC, is a first step in that direction, but years of effort are still needed to bring linear electron colliders to the same state of development as circular proton colliders.

One machine is being constructed that lies between the categories discussed above. The DESY laboratory in Hamburg has just begun construction of an electron-proton collider called HERA. It will have $820 \mathrm{GeV}$ protons colliding with $30 \mathrm{GeV}$ electrons with a luminosity of about $5 \times 10^{31} \mathrm{~cm}^{-2} \mathrm{~s}^{-1}$.

The SSC will build on the experience of earlier hadron-hadron colliders, especially the Tevatron Collider, just now coming into operation with its high energy and its superconducting magnets. The SSC represents an enormous step in both energy and luminosity over existing machines. In the energy regime of interest only a proton-proton machine is today capable of the high luminosity necessary to gather information on the rarest and possibly most interesting phenomena. The very high energy of the SSC will take it to a completely unexplored domain, providing answers to some of the fundamental questions posed above and undoubtedly uncovering new, unanticipated phenomena. 
Table Ia

HAORON-HADRON COLLIDERS

\begin{tabular}{|c|c|c|c|c|}
\hline Name & $\begin{array}{l}\text { Energy } \\
\text { (GeV) }\end{array}$ & $\begin{array}{l}\text { Particle } \\
\text { Types }\end{array}$ & $\begin{array}{l}\text { Luminosity } \\
\left(\mathrm{cm}^{-2} \mathrm{~s}^{-1}\right)\end{array}$ & Status \\
\hline $\begin{array}{l}\text { ISR } \\
\text { (CERN, Geneva) }\end{array}$ & $\begin{array}{l}30+30 \\
30+30\end{array}$ & $\begin{array}{l}p p \\
p \bar{p}\end{array}$ & $\begin{array}{l}10^{31}-10^{32} \\
\sim 10^{28}\end{array}$ & $\begin{array}{l}\text { completed } 1971 \\
\text { decommissioned } 1984 \\
1981-1982\end{array}$ \\
\hline $\begin{array}{l}\text { Sp } \bar{p} S \\
\text { (CERN, Geneva) }\end{array}$ & $370+310$ & $\mathbf{p} \bar{p}$ & $>10^{29}$ & completed 1981 \\
\hline $\begin{array}{l}\text { Tevatron } \\
\text { (FNAL, USA) }\end{array}$ & $1000+1000$ & $p \bar{p}$ & $\frac{10^{30}}{\text { (design) }}$ & $\begin{array}{l}\text { first test } 1985 \\
\text { operational in late } 1986\end{array}$ \\
\hline $\begin{array}{l}\text { UNK } \\
\text { (Serpukhov, } \\
\text { USSR) }\end{array}$ & $400+3000$ & $\mathrm{pp}$ & $\begin{array}{l}10^{32} \\
\text { (design) }\end{array}$ & $\begin{array}{l}\text { completion projected for } \\
1990 \text { 's }\end{array}$ \\
\hline $\begin{array}{l}\text { LHC* } \\
\text { (CERN, Geneva) }\end{array}$ & $6000+6000 ?$ & $\mathrm{p} \overline{\mathrm{p}}$ & $\leq 10^{32} ?$ & 1990 's? \\
\hline SSC & $20000+20000$ & $p p$ & $10^{33}$ & $\begin{array}{l}\text { proposed for completion } \\
\text { in } 1994\end{array}$ \\
\hline
\end{tabular}

*Currently under discussion as one possible future option at CERN. 
Table Ib

ELECTRON-POSITRON COLLIOERS

\begin{tabular}{|c|c|c|c|}
\hline Name & $\begin{array}{l}\text { Energy } \\
(\mathrm{GeV})\end{array}$ & $\begin{array}{l}\text { Luminosity } \\
\left(\mathrm{cm}^{-2} \mathrm{~s}^{-1}\right)\end{array}$ & Status \\
\hline $\begin{array}{l}\text { SPEAR } \\
\text { (SLAC, USA) }\end{array}$ & $4+4$ & $-10^{31}$ & operating since 1972 \\
\hline $\begin{array}{l}\text { DORIS } \\
\text { (DESY, Hamburg) }\end{array}$ & $5+5$ & $\sim 10^{31}$ & operating since 1974 \\
\hline $\begin{array}{l}\text { VEPP-4 } \\
\text { (Novosibirsk, } \\
\text { USSR) }\end{array}$ & $6+6$ & $\sim 10^{30}$ & $\begin{array}{l}\text { initial operation in } 1979 \\
\text { (at lower energies) }\end{array}$ \\
\hline $\begin{array}{l}\text { CESR } \\
\text { (Corne 11, USA) }\end{array}$ & $8+8$ & $\sim 3 \times 10^{31}$ & operating since 1979 \\
\hline $\begin{array}{l}\text { PEP } \\
\text { (SLAC, USA) }\end{array}$ & $18+18$ & $\sim 3 \times 10^{31}$ & operating since 1980 \\
\hline $\begin{array}{l}\text { PETRA } \\
\text { (DESY, Hamburg) }\end{array}$ & $22+22$ & $\sim 3 \times 10^{31}$ & operating since 1978 \\
\hline $\begin{array}{l}\text { TRISTAN } \\
\text { (KEK, Japan) }\end{array}$ & $30+30$ & $\sim 10^{31}$ (design) & $\begin{array}{l}\text { scheduled for completion } \\
\text { in } 1986\end{array}$ \\
\hline $\begin{array}{l}\text { SLC } \\
\text { (SLAC, USA) }\end{array}$ & $50+50$ & $>10^{30}$ (design) & $\begin{array}{l}\text { scheduled for completion } \\
\text { in early } 1987\end{array}$ \\
\hline \multirow[t]{2}{*}{$\begin{array}{l}\text { LEP } \\
\text { (CERN, Geneva) }\end{array}$} & $50+50$ & $\sim 10^{31}$ (design) & $\begin{array}{l}\text { scheduled for completion } \\
\text { late } 1988\end{array}$ \\
\hline & $100+100$ & & $\begin{array}{l}\text { energy upgrade of the } \\
\text { facility planned for } \\
\text { early } 1990 \text { 's }\end{array}$ \\
\hline
\end{tabular}




\subsection{SYNCHROTRON COLLIDERS}

The increasingly large facilities described in the previous section are the focal points of the research in high energy physics. The history of the field is dominated and paced by their ability to open up new energy domains for experimentation. To understand the nature of the SSC it is useful to describe in elementary terms the workings of the various components of any modern accelerator-storage ring complex. The treatment here will orient the unfamiliar reader for the subsequent technical discussion.

At the SSC experimenters will study the collisions of oppositely directed bunches of protons. As discussed in Section 1.5, such collisions are much more efficient in utilizing the energy of the beam particles than the bombardment of a stationary target in the laboratory. The bunches will be slim cylinders 10 micrometers or less in radius and about 15 centimeters in length along the line of collision. Each bunch will contain about 1010 protons. Because of the small probability for proton loss during the collision, these bunches can pass through each other repeatedly for many hours without need for replenishment. This is accomplished in a storage-ring collider by causing streams of bunches to counter-circulate on closed paths which intersect head-on, or at a small angle, at the desired interaction points. Several such colliders, based on the synchrotron principle, have been built. While it will attain a beam energy 20 times the highest achieved so far, the design of the SSC will be similar to previous machines.

The synchrotron accelerator is a direct descendant of the cyclotron, using magnetic fields to guide the charged particles in a closed path and repetitive acceleration to increase their energy, but incorporating essential discoveries made in the 1940's and 1950's. A synchrotron has three basic sub-systems: (1) A source of the charged particles, which usually includes some early acceleration before injection into the synchrotron, (2) a magnetic confinement system, and (3) an accelerating system. These are shown schematically in Figs. $1-3$ and $1-4$.

(1) Source

The basic source of protons to be accelerated is a small volume of ionized hydrogen gas from which the charged nuclei (protons) are extracted by suitably arranged electrodes and emerge with kinetic energies of a few keV (Fig. 1-4, top left). Such a beam is slow moving and quite divergent. For these reasons it is common practice to preaccelerate the beam from the ion source before introducing it into the synchrotron. This is accomplished initially by static electric fields and then by one or more linear accelerators whose radiofrequency electric fields cause the accelerated particles to cluster in bunches as they gain energy (Fig. 1-4, top center and top right). Typically the beam is carried to an energy of several hundred million electron volts (MeV) before transfer to a synchrotron.

(2) Confinement System

The deflection of a charged particle by a magnetic field provides the mechanism for steering and confining a beam as its energy is increased or as 


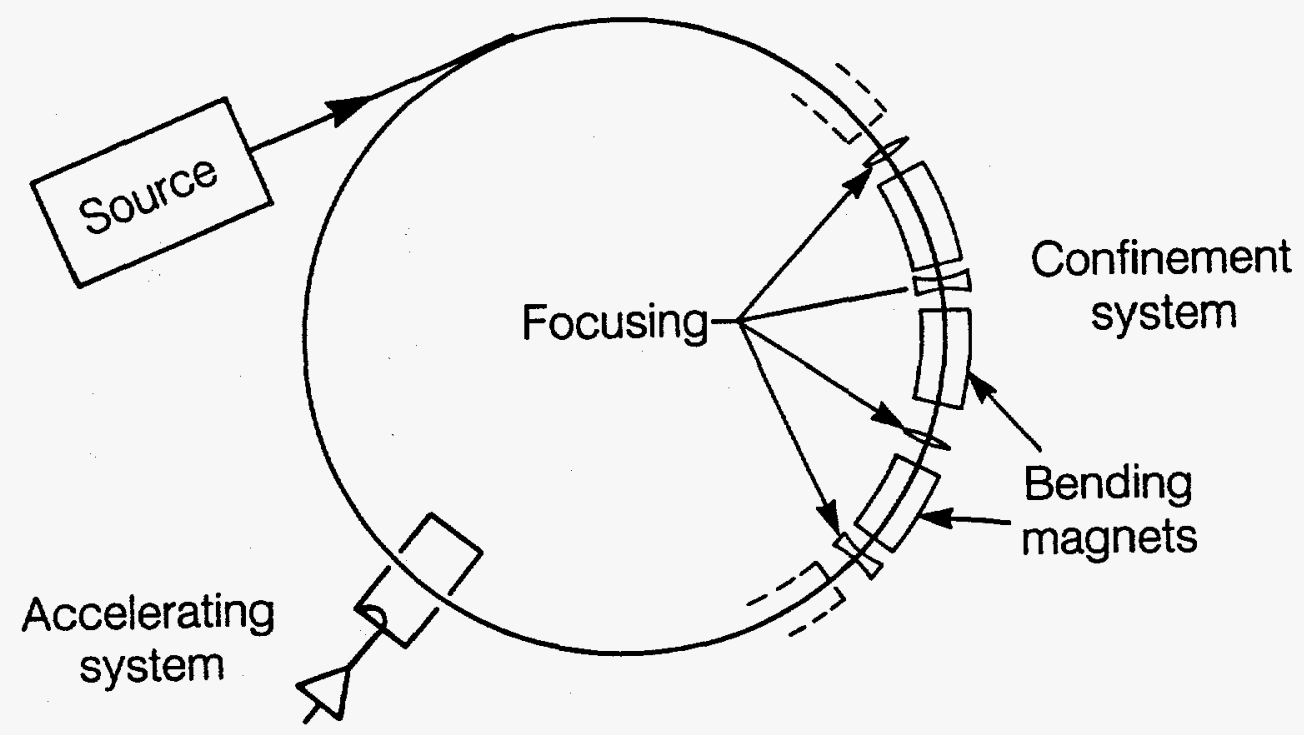

(a)

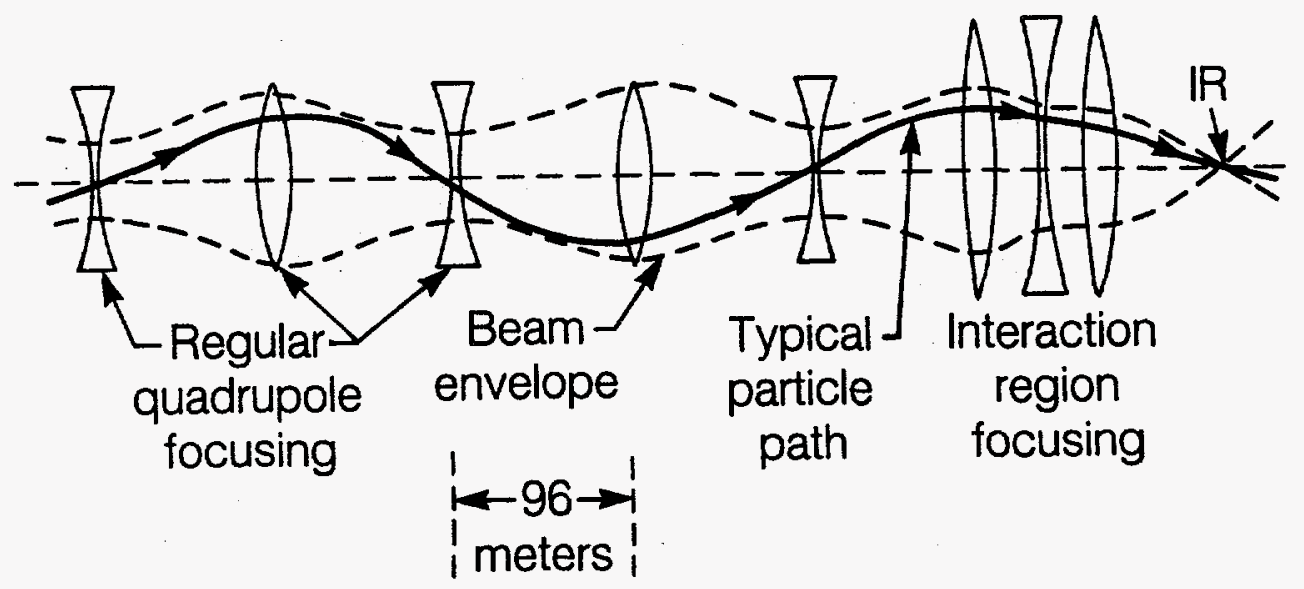

(b)

Figure 1-3. (a) Schematic diagram of the major components of a modern synchrotron. (b) Diagram of the focusing parts of the magnetic confinement system. Optical lens symbols are used for the magnetic elements. In the SSC, each "lens" is actually a quadrupole magnet over 3 meters long. Not shown between successive quadrupoles are the dipole bending magnets. For the SSC, the quadrupole separation is 96 meters $(315 \mathrm{ft}$ ), with room for 5 bending magnets. 


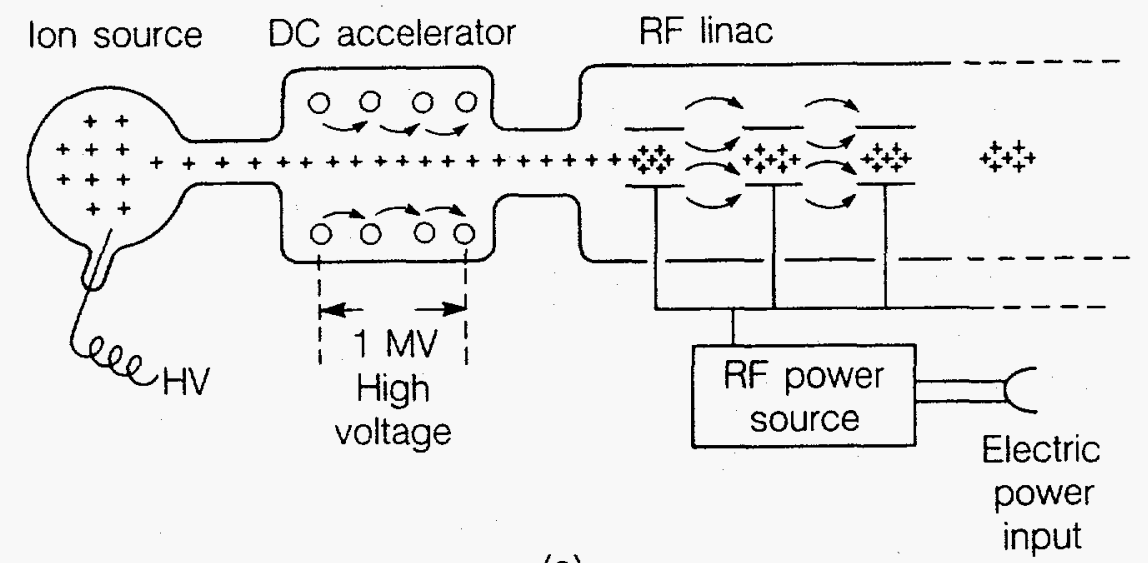

(a)

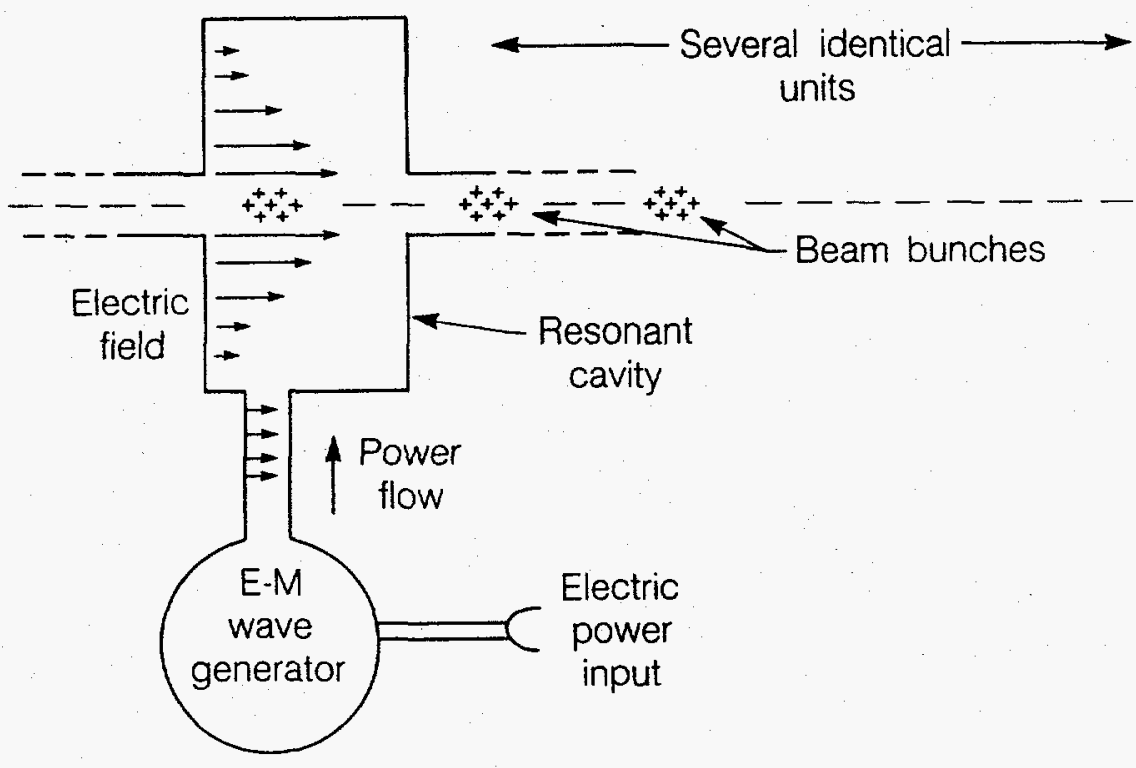

(b)

Figure 1-4. (a) Schematic diagram of the proton source and early stages of acceleration. Ions are accelerated electrostatically through approximately 7 million volts, and then by radio-frequency fields in one or more linear accelerators. (b) Sketch of one of the main ring radio-frequency accelerating cavities. The proton bunches pass through the cavity at the right time to be accelerated by the oscillating electric field. 
it is stored at fixed energy. The bending radius of a magnetic guide field is related to the energy of the particle and the field strength according to

$$
R(k m)=\frac{10 E(T e V)}{3 B(\text { tes la })}
$$

When allowance is made for the spaces between bending magnets for focusing magnets and other equipment, the physical radius of an accelerator can be up to $50 \%$ larger.

The language of optics is used to describe the deflection and focusing of particle beams. The dipole bending magnets are the prisms, while the quadrupole focusing magnets are the lenses of the beam-optical system. The dipoles have a uniform magnetic field perpendicular to the plane of the synchrotron ring and produce a constant deflection of the beam in the plane. The stronger the field, the smaller is the radius of curvature, for a fixed particle energy. The focusing quadrupole magnets are so constructed that the magnetic field strength is zero at the very center line and grows in proportion to the distance from the axis. Particles passing exactly along the equilibrium orbit then are undeflected in traversing the focusing magnet. Particles traversing it off axis are deflected in proportion to how far off center they are. By this proportionality of deflection to displacement, the particles are focused into a beam of the needed size.

As indicated with the use of concave and convex lens symbols in Fig. 1-3, the focusing magnets are alternately focusing and defocusing. (Actually, things are a bit more complicated. A "focusing" magnet (convex lens symbol) focuses particles in, say, the horizontal direction and defocuses them in the vertical direction, while a "defocusing" magnet (concave lens symbol) does the opposite.) By alternating the sense of the focusing magnets, beams can be focused simultaneously in both directions This scheme was discovered in the $1950^{\prime} \mathrm{s}$ to produce much smaller excursions or oscillations of the particles away from the equilibrium orbit and permit smaller beam tubes and smaller magnets than in the weaker focusing scheme then in use. Near the points where the beams are to collide, extra strong lenses are used to focus the beam to a very high density thereby enhancing the luminosity and reaction rate, as indicated schematically in Fig. 1-3, bottom right.

This scheme of separate bending and focusing magnets is remarkably tolerant to alignment errors as the equilibrium orbit tends to follow the centers of the focusing magnets. Over a length of about $1 \mathrm{~km}$ along the orbit, alignment errors of individual focusing magnets centers need be better only than about 0.5 to $1 \mathrm{~mm}$. On a larger scale, drifts in the average position of the centers can be considerably more, subject, of course, to the condition that the ring close on itself.

As the particles orbit around the machine, they undergo oscillations about an equilibrium orbit, the nature of which depends on their energy and the specifics of the arrangement of the many bending and focusing magnets along the ring. The magnet arrangement is called the lattice of the synchrotron; the state of motion of the oscillations is described by the "tune." The tune is actually a pair of numbers, like 82.42 and 82.38 , that indicates the number 
of cycles of oscillation in the horizontal and vertical directions the particles undergo in one turn around the machine. It is a feature of the alternating scheme of focusing that the tune must be picked carefully so that the powerful focusing features of the scheme are not spoiled by resonant blow-up of the beam.

The reason for discussing a technical point like the tune is that it can be altered ("shifted", and so "tune shift") because the counter-rotating beams pass through each other at the interaction points. The shift of tune occurs because the electromagnetic fields of one bunch act on the particles in the other as an additional effective focusing magnet in the lattice. Each beam experiences an effective lattice different from the one it would see if it were the only beam in the machine. If the beam currents are too large, the tune shift is sufficient to cause resonant growth of the transverse size of the beams. The so-called beam-beam tune shift is one of the factors limiting the peak luminosity of the collider.

\section{(3) Accelerating System}

Acceleration, or increase of the beam's energy along the orbit, is accomplished by transformer action, indicated schematically in the bottom of Fig. 1-4. The beam particles typically gain an energy of several MeV each time they pass through the acceleration system. The electric current of the beam flows in the secondary circuit of the transformer while the primary is driven by a radiofrequency cavity whose dimensions are adjusted so that it resonates electrically at the driving frequency. The frequency must be a multiple of the rotation frequency of particles in the ring in order that they receive the proper acceleration each time they come around. An arrangement appropriate for the SSC has component parts quite similar to those used in electron colliders. [See Fig. 1-5] Modern acceleration systems are so efficient that the entire cavity array for the SSC main ring will occupy a length of only $25 \mathrm{~m}$ along its orbit, one such system for each ring.

\section{(4) Cascade of Accelerators}

Given the basic sub-systems, how does a modern synchrotron accelerator function? Particles from the source, after acceleration to several hundred MeV kinetic energy in a linear accelerator, are transferred to a synchrotron. During this injection phase the magnetic field of the synchrotron's confinement system is held constant at the value corresponding to the injection energy and the radius of the ring. When the requisite number of protons have been transferred, the magnetic field is increased and the beam energy grows in proportion through the action of the acceleration system. If the synchrotron is to be used as a collider, the magnetic field is increased to a value corresponding to the desired beam energy and then held constant for many hours, even days, while the beams collide and slowly attenuate. When the reaction rate has become too small to be optimal, the beam is extracted from the ring and the acceleration and storage process is repeated.

The protons that make up the beam inevitably have some natural divergence, that is, they have some small component of momentum transverse to the direction of the desired orbit and require focusing. The ratio of this small transverse component to the momentum along the orbit is the angle which the particle trajectory makes with the wanted path. As the momentum along the 


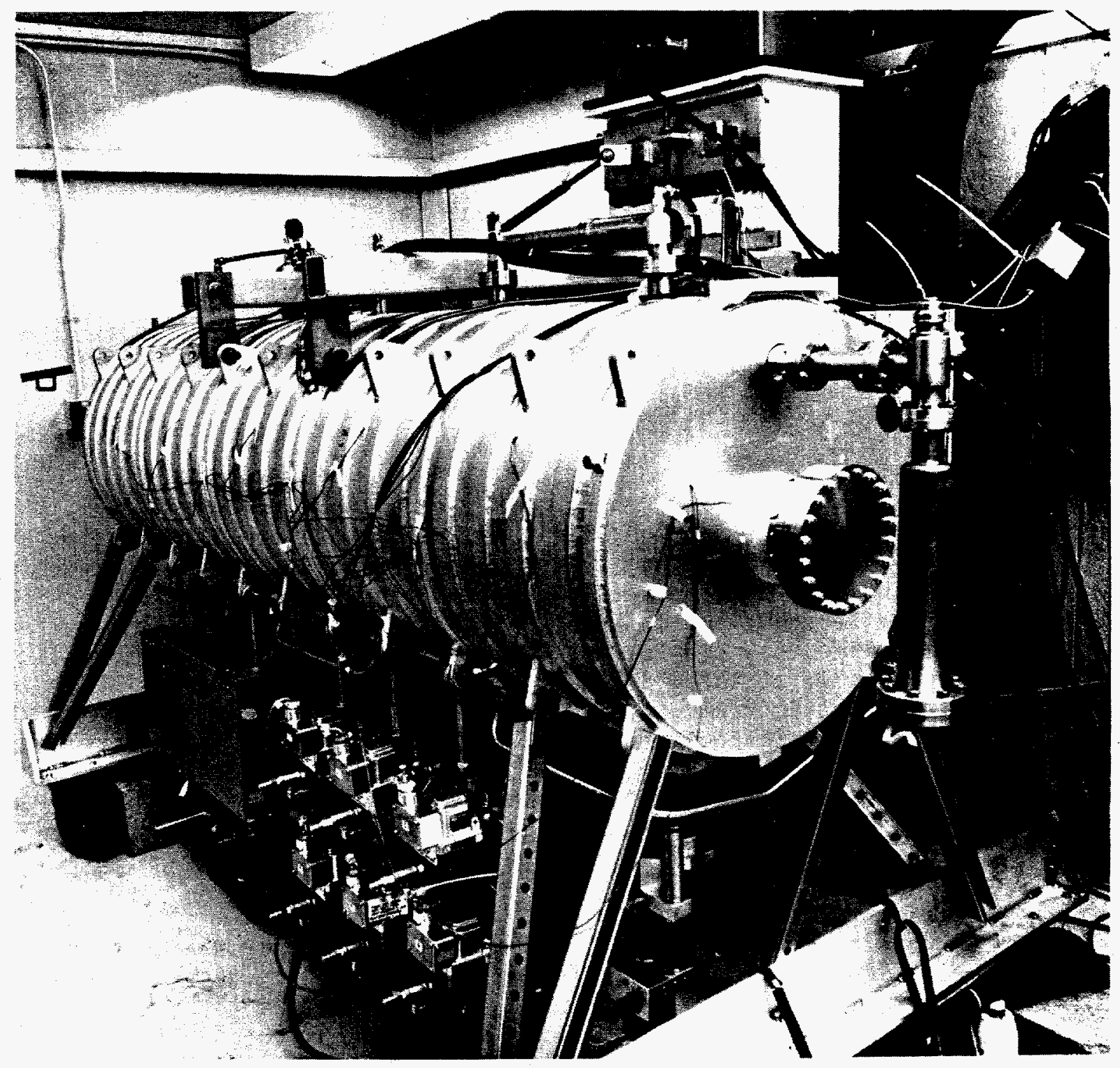

Figure 1-5. One of the rf cavities of the accelerating unit of the PEP electron-positron accelerator-storage ring at Stanford University. The SSC main ring unit will be essentially the same. 
equilibrium orbit is increased by the acceleration system, this angle decreases, which, together with the increasing magnetic field, results in a decrease in the transverse beam size compared to that at injection. Because an important part of the cost of the confinement system is proportional to the volume occupied by magnetic field, for high energy synchrotrons it is economically advantageous to use a series of synchrotrons with successively increasing top energies and decreasing apertures, rather than a single synchrotron to accomplish the full span of acceleration required. For this and other technical reasons the ratio of top energy to injection energy of a synchrotron is usually designed to be somewhere between 10 and 100 , although higher ratios have been used successfully. The large proton accelerators at Fermilab and at CERN, Geneva each consist of a cascade of accelerators, and so will the SSC, as discussed in detail in Chapters 4 and 5.

(5) Fermilab's Accelerator and Collider

The complex of accelerators at Fermilab provides a specific illustration of the principles just discussed. The Fermilab main ring has a physical radius of $1 \mathrm{~km}$ and a maximum energy in its conventional (copper and iron) magnet system of $0.45 \mathrm{TeV}$ at a peak field of approximately $2.2 \mathrm{Tes}$ la. An aerial view of the Fermilab accelerator is shown in Fig. 1.6. The cooling ponds and surface buildings of the main ring are clearly visible. At the left can be seen a high-rise laboratory building. In the foreground is the circular ring of the rapid-cycling booster synchrotron, with the source and linear accelerator feeding it from the left rear. The booster synchrotron receives protons of $200 \mathrm{MeV}$ kinetic energy from the $145 \mathrm{~m}-10 \mathrm{ng}$ linear accelerator and accelerates them to $8 \mathrm{GeV}$. The $8 \mathrm{GeV}$ protons enter the main ring where, in pre-Tevatron days, they were accelerated typically to $400 \mathrm{GeV}$ before being extracted and dispatched to fixed targets in the experimental areas, shown stretching away from the left side of the main ring in Fig. 1-6.

In some detail, the sequence of acceleration and extraction for fixed target operation of the original Fermilab accelerator was typically as follows: Protons from the linear accelerator are injected into the booster synchrotron in a few milliseconds, a time short compared to the 33 millisecond acceleration time from $200 \mathrm{MeV}$ to $8 \mathrm{GeV}$. The $8 \mathrm{GeV}$ beam is transferred to the ma in ring, whose guide field is being held to accept $8 \mathrm{GeV}$ protons. The booster ramps its magnetic field down in $33 \mathrm{~ms}$, accepts more protons from the linac, and accelerates them to $8 \mathrm{GeV}, 66 \mathrm{~ms}$ after the first batch. The booster acceleration cycle thus occurs at a rate of 15 times per second. In $0.8 \mathrm{~s}$, the booster can inject 13 batches into the quiescent main ring. The 13 batches nearly fill the main ring and correspond to perhaps $10^{13}$ protons. The main ring acceleration cycle now begins and the confining field increases linearly in time in step with the acceleration process until full field and full energy $(400 \mathrm{GeV})$ is reached in 4 seconds. The magnets are then held at full field and the beam extracted during typically 2 seconds. The magnets are ramped down to injection field again, the overall cycle lasting about 10 seconds.

During the past five years the Fermilab accelerator has been modified by the addition of a ring of superconducting magnets in the main ring tunnel for the purpose of doubling the maximum energy of the proton beam and at the same time saving power. Most recently, an antiproton source has been constructed 


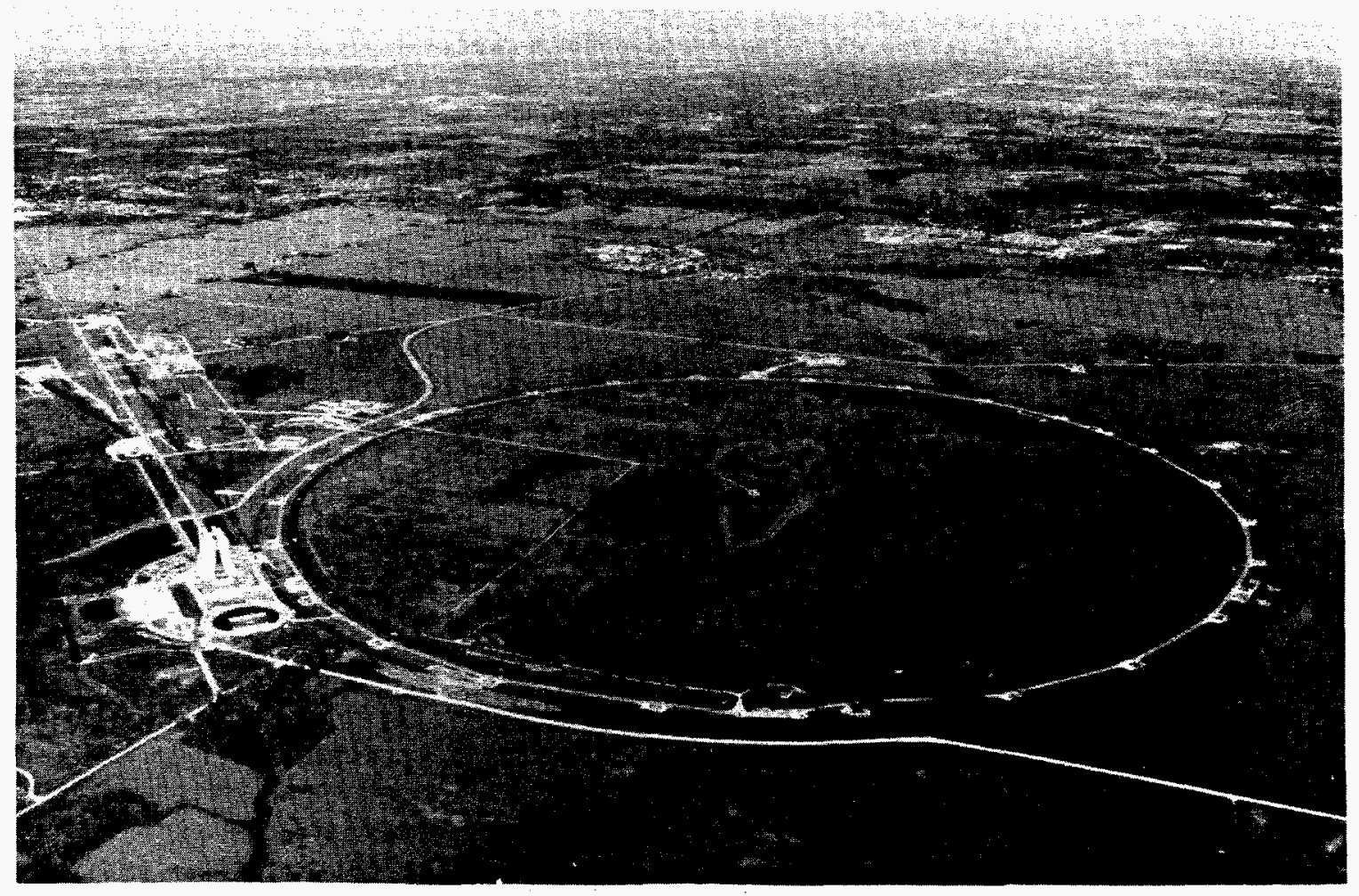

Figure 1-6. Aerial view of the Fermilab accelerator complex. The large circle outlines the underground main ring. The smaller circle at the left is the booster synchrotron, fed by a linear accelerator. The high-rise laboratory building is behind the linac-booster area. 
to give collider, as well as fixed target, capability. The Tevatron, as the augumented complex is called, now provides $0.8 \mathrm{TeV}$ proton beams and will soon operate in the collider mode with counter-rotating 0.8-1.0 TeV beams of protons and antiprotons. As discussed in Chapter 3, the Tevatron complex provides an impressive first example of the successful large-scale application of superconducting magnet technology to accelerators.*

In its collider mode, the Tevatron is a cascade of three synchrotrons, not counting the antiproton source--the booster (200 MeV to $8 \mathrm{GeV}$ ), the old main ring $(8 \mathrm{GeV}$ to $150 \mathrm{GeV})$, and the new superconducting ring (150 GeV to $1 \mathrm{TeV}$ ): The SSC will be a similar cascade, as described in Chapters 4 and 5 , with the addition of the main SSC collider ring ( $1 \mathrm{TeV}$ to $20 \mathrm{TeV}$ ). The cycling of the lower energy synchrotrons will be similar to that described above, with the last booster being superconducting like the Tevatron, with an acceleration time of about 15 seconds to 1 TeV and a repetition time of 40 seconds. Filling of the two main rings will take 30 minutes, followed by an acceleration time of 15 minutes to $20 \mathrm{TeV}$. Collisions for physics then occur with the stored beams for periods of a day or more, before repeating the cycle.

* Superconducting magnet technology for accelerators is discussed by R. Palmer and A.V. Tollestrup, Annual Review of Nuclear and Particle Science, 34, 247(1984). The Tevatron itself is described in detail by H.T. Edwards, Annual Review of Nuclear and Particle Science, 35,605 (1985). 



\section{CHAPTER 2}

\section{SCIENTIFIC NEED FOR THE SSC}

The results of the experiments conducted thus far at the CERN SPS Collider confirm a simple picture of high energy proton collisions. The protons are composed of three fundamental quarks, plus a mixture of quarks, antiquarks, and gluons. The most interesting collisions of protons occur when a single parton (quark, antiquark, or gluon) from one proton collides with a single parton from the other. Thus the total energy in the basic collision is considerably less than that apparently available from the two beams. It is the fundamental interactions of the partons that we wish to study.

At the CERN Collider, clear signals have been seen for the scattering of partons through large angles. One process of this sort is illustrated in Fig. 2-1. It shows one quark from each proton interacting by exchanging a gluon. The exiting quarks carry a large energy transverse to the direction of the beams. These quarks turn into observable pions, kaons, protons, etc. If a quark from one proton and an antiquark from the other collide, they might produce a $W$ or a $Z$. It is this sort of event that provided the evidence for these particles.

\subsection{PHYSICS AT SSC ENERGIES}

At the SSC, the same basic mechanisms would be at work. The energy available in the collision of the partons would be enormously greater, however, than in the collisions observed in any earlier machine. These collisions could thus produce new particles, never before observed. What might these particles be? Based on our understanding of fundamental interactions to date, we can make some speculations. The list provides some guideline for phenomena at the SSC :

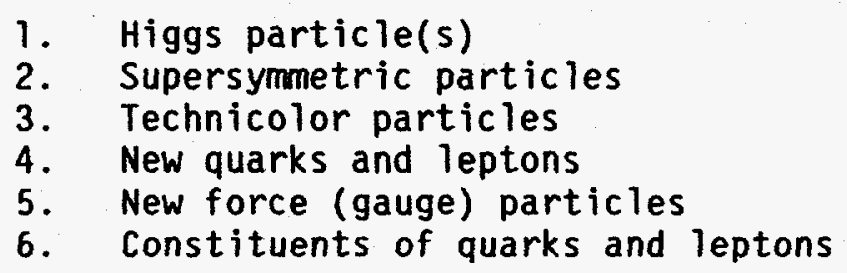

The first three items on the list are related to the origin of the masses of particles. As mentioned in Sect. 1.4, the standard model begins as a theory of massless particles. The quarks, charged leptons, $W$, and $Z$ get masses through their interaction with an omnipresent field. The first and simplest version of the model contains a single additional particle, the Higgs particle, that is intimately connected with this mass-generating field. Assuming it exists, we can predict reliably all its properties except one: its mass. If it is light enough (less than about $35 \mathrm{GeV}$ ), it should be observed in $Z$ decays studied at SLC or LEP. The SSC and other multi-TeV hadron colliders are suited to finding it should the mass of the Higgs particle lie above $200 \mathrm{GeV}$. 


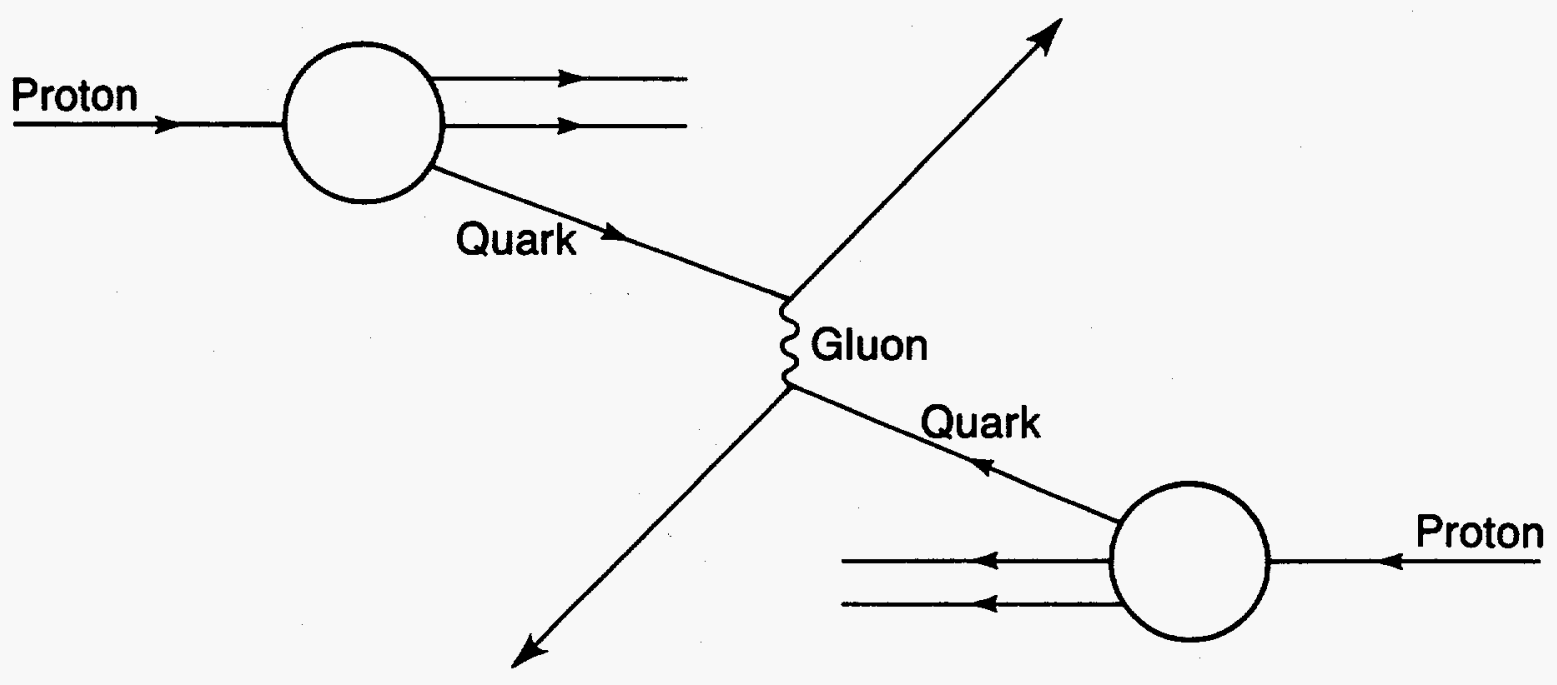

XBL 8512-12797

Figure 2-1. Parton-parton scattering. The constituents inside the proton (called partons) share the proton's momentum. In a high-energy collision between protons, it is actually the partons that collide. The example shown is quark-quark scattering by gluon exchange. 


\section{(1) Higgs Particle}

The Higgs particle is not stable, but decays into other particles. Because it is related to the origin of masses, it turns out that it is most likely to decay into very massive particles. If the Higgs mass is greater than twice the mass of the $W$ or $Z$, it will decay into two $W^{\prime} s$ or 2 Z's. Some of these decays could be detected. If enough events of this sort are accumulated, the Higgs particle could be identified.

Although the mass of the Higgs particle cannot be predicted, there are powerful general arguments that demonstrate that the phenomena associated with the Higgs particle must manifest themselves at an energy less than about 1 TeV. A similar argument was used some years ago to show that some new phenomenon had to occur below about $300 \mathrm{GeV}$ for the case of weak interactions. That new phenomenon turned out to be the $W$ particle found at a mass of $83 \mathrm{GeV}$. The argument comes simply from calculating predictions in a theory without the Higgs particle (or in the earlier instance, without the $W$ particle). At some high energy, here $1 \mathrm{TeV}$, the calculations predict that some process must occur with a probability greater than $100 \%$. This absurd result shows that something has been omitted from the calculation: there must be some additional phenomenon that enters before this energy is reached.

This argument is quite general. It applies even if the simplest model, which has a single Higgs particle, is not correct and instead there are some other particles that play the role of the Higgs particle. Therefore, the scale of $1 \mathrm{TeV}$ is one that is singled out as a target for the SSC, just as a lower energy scale was singled out in the search for the $W$. The task here is much more difficult than it was for finding the $W$ and $Z$. There it was possible to make precise predictions for the masses. That is not possible at present for the Higgs particle.

The Higgs particle may be difficult to find. However, a number of extensions of the standard model have alternative mechanisms to give rise to particle masses. In these extensions there are other particles besides the Higgs particle that would reveal themselves. Most of these particles would be much easier to find.

\section{(2) Supersymmetry}

One extension is called supersymmetry. In this model there are several Higgs particles to look for. This makes the task easier. There are, besides, many other new particles that would be produced at the SSC if supersymmetry plays a role in the generation of the masses of the known particles.

Supersymmetry proposes that there are connections between particles with angular momentum one-half (e.g. the quarks and leptons), particles with angular momentum one (e.g. the force particles), and particles with zero angular momentum (e.g. the Higgs particle). For every quark or lepton there must be a "supersymmetric" partner with spin zero, called squarks and sleptons. For every force particle, there should be a partner with angular momentum onehalf: for the photon a photino, for the gluon a gluino, for the $W$ a Wino, and for the $Z$ a Zino. 
Where are these particles? None has been found to date, but if supersymmetry is connected to mass generation, these particles cannot be too much heavier than the known particles, surely less than a few TeV. If they exist, many of them should be produced at the SSC.

While no experimental evidence of supersymmetry has been found, the theory has great attractiveness. It provides a partial answer to the hierarchy problem. In most theories, it is hard to prevent the mass of the Higgs particle from increasing up to the highest mass scale available, the Planck mass. In supersymmetric models, the mass of the angular momentum zero particles is not. so pathological. The reason is that when one calculates the mass, for every contribution pushing the mass up, there is one of equal strength, pushing down.

Supersymmetry has other virtues. It is possible to incorporate gravity into the model and unify all four forces. Indeed, recent theoretical advances have produced models that incorporate all the forces and avoid the devastating problems that have previously plagued theories of quantum gravity. These new models are based on "superstrings". The basic units in the theory are not point particles, but strings of fantastically small dimensions. Superstring theories look very promising and enormously challenging. Efforts are being made to derive predictions that could be tested with accelerators including the SSC.

\section{(3) Technicolor}

The term technicolor was coined to describe models in which there are forces analogous to the color forces carried by the gluon, but which are characterized by a mass scale ( $1 \mathrm{TeV}$ ) set by the $W$ and $Z$ masses, rather than the $1 \mathrm{GeV}$ mass scale of ordinary hadrons. These hypothetical forces would act between techniquarks, particles analogous to ordinary quarks. States of techniquarks and anti-techniquarks bound together by the technicolor force could play the role of the Higgs particle in the simple theory described above. Because such Higgs particles are not fundamental, but are composed of other particles, some of the technical difficulties of the simple model can be avoided. Technicolor models predict a great variety of new particles that could be observed at the SSC.

\section{(4) New Quarks and Leptons}

In addition to new particles associated with the problem of mass, there may be new quarks and leptons that can be discovered at the SSC. So far, three generations of quarks and leptons have been discovered (see Fig. 1-2, p.8.), but there is no assurance that there are not more. Important evidence will come from the study of $Z$ decays at the SLC and LEP. By measuring $Z$ decays, it is possible to infer how many neutrinos there are, assuming, as is so far the case, that all neutrinos are massless (or at least very light). It is possible that quarks and leptons come in more complex arrangements than the families we have seen thus far. Perhaps there are additional quarks even with charges in a different pattern than those observed up to now. Another possibility is that there are additional leptons and that both the charged and neutral ones are very massive. Such particles could be observed at the SSC. 
(5) New Force (Gauge) Particle

Perhaps more dramatic than the discovery of a new quark or lepton would be the discovery of a new force particle. Each force particle represents a specific force: the photon, the electromagnetic force; the gluon, the strong force; the $W$ and $Z$, the weak force. Thus a new force particle would indicate a new force, or at least a complication of an existing law of force. This would be quite startling because the four known forces were already apparent early in this century. A new force particle would be observed just as the $W$ and $Z$ were at CERN. In a high energy collision a quark and an antiquark could annihilate to form the force particle which would subsequently decay. Its decay into a pair of light leptons would be an especially clean signature.

(6) Quark and Lepton Sub-structure

So far as we know at present, the quarks and leptons are fundamental particles in the sense that they do not appear to be made of more fundamental constituents, of course, we may have failed to find the constituents inside them only because we have not probed deeply enough. The constituents of the proton only revealed themselves when SLAC was able to direct its high energy. electron beam on protons. Previous experiments at lower energy were incapable of resolving the details inside the protons and neutrons. The SSC will provide the means to probe more deeply than ever before inside matter and may reveal a new layer beneath the quarks and leptons.

This partial list of possible discoveries - Higgs particles, supersymmetric particles, technicolor particles, etc. - cannot be more than a guess. Past experience suggests that a new accelerator opening up a vast new energy range will make discoveries that could not be anticipated in advance. It is these discoveries that are often the most important ones. Still, the tentative list demonstrates that the reach of the SSC will encompass issues fundamental to our understanding of the basic constituents and forces of matter, and especially important to the understanding of the origin of mass.

(7) Connections to Other Scientific Fields

While particle physics continues to probe more and more deeply into matter and deals with higher and higher energy, the issues at stake have not become narrower. On the contrary, the concerns of particle physics are becoming more and more entwined with those of neighboring fields. This is especially true of astrophysics, condensed matter physics, and nuclear physics.

Cosmology and particle physics share a common frontier: interactions at very high energy. At the beginning of the universe, according to our current picture, the energies of the existing particles were extremely high. The exotic particles studied in high energy accelerators existed in great numbers. By studying the highest energy reactions, we are studying the conditions that prevailed in the earliest fraction of a second of the universe. Moreover, some of these exotic particles may have persisted to this day. There is evidence that much of the mass of the universe is not present in the stars or ordinary matter between them. It may be that it is in the form of yet unobserved fundamental particles. These particles could be related to supersymmetry or some other theory that could be tested at the SSC. 
In studying the phenomenon that confines the quarks inside observed particles and prevents the quarks from being seen individually, particle physicists have had to confront phase transitions. In the generation of particle masses in the electroweak theory, there is also a phase transition, this one analogous to that occurring inside a superconductor. The field of phase transitions has been an area of fruitful interaction between particle physics and condensed matter physics. Phase transitions also play an essential role in our understanding of the early moments of the universe.

Study of the phase transition that causes quarks to be confined inside observed hadrons may be possible in nuclear collisions between relativistic heavy ions. Collisions between such large aggregates have the potential of occasionally providing enough energy density over a large enough region to cause momentary creation of a quark-gluon plasma, rather than a collection of very energetic, but ordinary, particles. This rare deconfined phase should exhibit properties different from the usual collisions, thereby throwing light on collective aspects of the strong force not accessible in elementary particle collisions.

The SSC, through its investigation of fundamental physical processes, will provide new insights into these questions of great significance to other sciences as well as particle physics, and to our general appreciation of the world in which we live. It will be a powerful tool for extending those investigations of matter that have led us to an understanding of the atom, the nucleus, and on to their smallest components.

\subsection{CONCEPTS OF VERY HIGH ENERGY COLLISIONS}

The energy range that can be explored by the SSC cannot be defined in a single statement. The range depends on the particular physics being explored. Each incoming proton is composed of partons (quarks, antiquarks, and gluons) that share the proton's momentum. The fundamental processes are the collisions of partons.

Figure 2-2 illustrates the production of a pair of new particles $Y$ and $\bar{Y}$. The process is described by the following equation for the interaction cross section,

$$
\sigma=\int d x_{1} d x_{2} f_{i}\left(x_{1}\right) f_{j}\left(x_{2}\right) \hat{\sigma}
$$

The elemental collision is that of two partons of types $i$ and $j$, which could be quarks or gluons depending upon the particular process. These partons have energies $x_{1}$ and $x_{2}$ times the energy of the incident proton beams. The quantity $f_{j}\left(x_{1}\right) d x_{j}$ is the probability that there is a parton of type $i$ with energy fraction between $x_{1}$ and $x_{j}+d x_{1}$ within a proton, and similarly for $f_{j}\left(x_{2}\right) d x_{2}$. These probabilities differ in detail, depending on parton type, but are largest when $x$ is small. The integral in the equation corresponds to summing over all the combinations of parton energies, weighted with the probability of occurrence. 


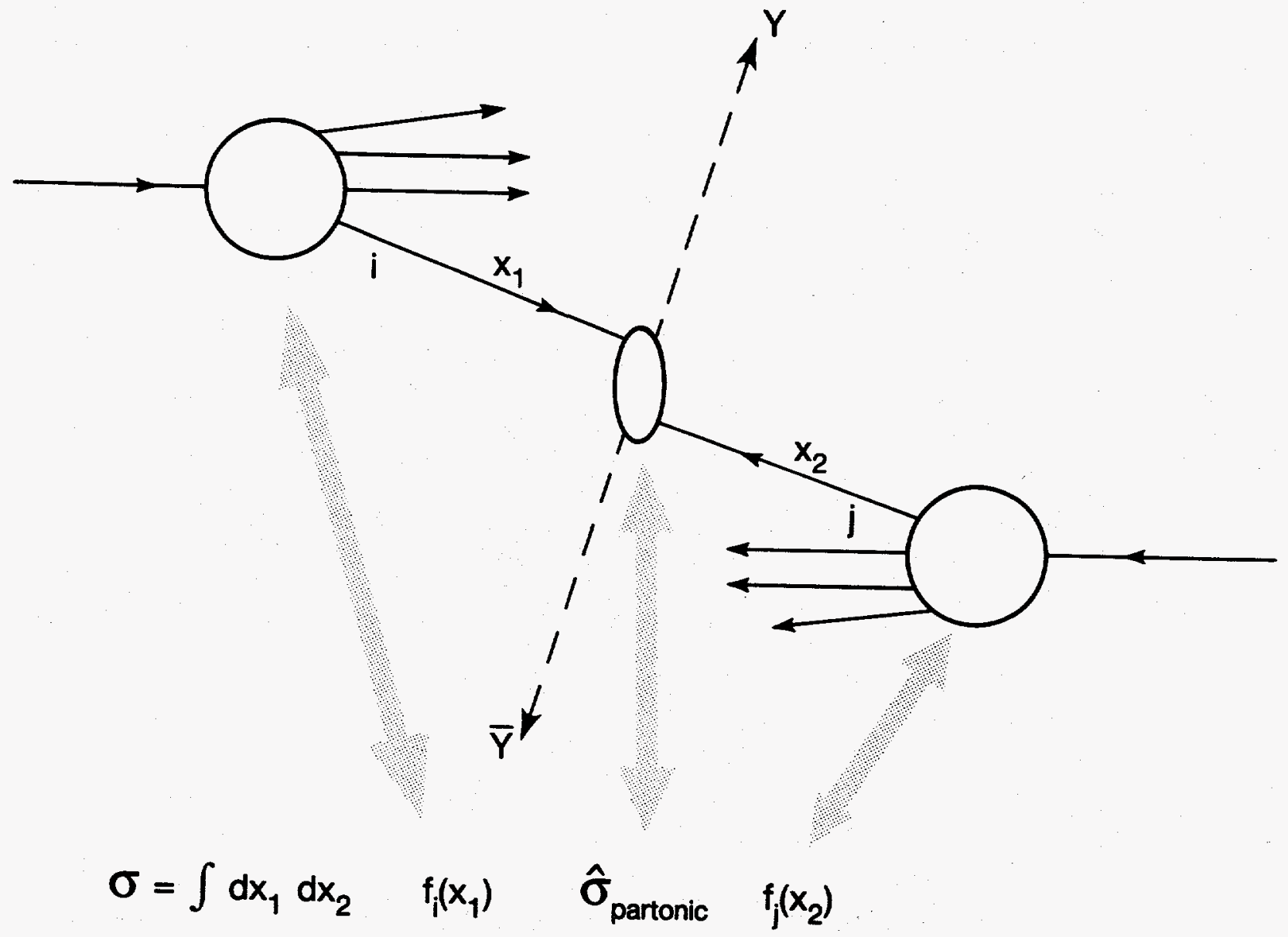

Figure 2-2. General parton-parton interaction. A parton of energy $x_{1} E$ in one proton collides with a parton of energy $x_{2} E$ from the other proton to produce a particle-antiparticle pair $Y \bar{Y}$ at large angles. The observed cross section is formed from the partonic cross section, folded with the respective probabilities of finding the specific partons of energies $x_{1} E$ and $x_{2} E$ within the two protons. 
The equivalent parton beam energy $\hat{E}$ flowing into the interesting process is related to the energy of the proton beam $E$ by

$$
\hat{E}=\sqrt{x_{1} x_{2}} E
$$

The probability of having a collision with useful total energy $2 \hat{E}$ in a collider of total energy $2 E$ is a rapidly falling function of $\hat{E} / E$, since $f_{i}\left(x_{1}\right)$ and $f_{j}\left(x_{2}\right)$ decrease as $x_{1}$ and $x_{2}$ increase.

The quantity $\hat{\sigma}$ is the cross section for the process of pair production of $Y \bar{Y}$ from incident partons $i$ and $j$. This cross section is easy to estimate. Incoming partons with energy $\hat{E}$ probe distances of order $1 / \hat{E}$; as the energy is increased, shorter distances are probed. The typical probed area or cross section is approximately $1 / \widehat{E}^{2}$. The exact value depends, of course, on the particular process. Nevertheless, this simple behavior illustrates the increasing importance of luminosity in high energy colliders. (Recall that the event rate is equal to the cross section times the luminosity).

The event rate for some new process can be written as the product of three factors, the actual luminosity of the collider, the effective luminosity for the type of collision (quark-quark, gluon-quark, etc) and the cross section for the parton process. The form of this effective luminosity can be deduced from the first equation above,

$$
\text { effective luminosity }=\int \frac{d x}{x} f_{i}(x) f_{j}\left(\hat{E}^{2} / E^{2} x\right) \text {. }
$$

The effective luminosity depends upon the energy of the beams of the parton process and upon the types of partons. It can be used to discuss the relative merits of different combination of beam energy and luminosity without recourse to specific theoretical models.

Figure 2-3 shows the behavior of this effective luminosity for gluon-gluon collisions for a variety of proton energies. A simple example will illustrate the utility of this figure. Suppose that we wish to investigate the production of a new quark of mass $1 \mathrm{TeV}$. Since this new quark must be produced in pairs the useful energy required is $2 \mathrm{TeV}$ at least. We can see that the effective luminosity at this energy is approximately a factor of 10 smaller in a collider with $10+10 \mathrm{TeV}$ beams than it is in one with $20+20 \mathrm{TeV}$ beams. We can therefore produce the same number of events in these machines only if the lower energy one has 10 times more luminosity. Such a rule of thumb is useful but should not be abused; there are cases where the lower energy machine is less effective even at higher luminosity because there are relatively more uninteresting events to obscure the sought-for signal.

It is interesting to note that at multi-TeV beam energies the parton contents of protons and antiprotons are very similar. Thus, except for minor details, multi-TeV collisions of $\bar{p}+\bar{p}, p+\bar{p}$ or $p+p$ all produce the elementary reactions we seek to study. Said another way: At equal luminosities and 


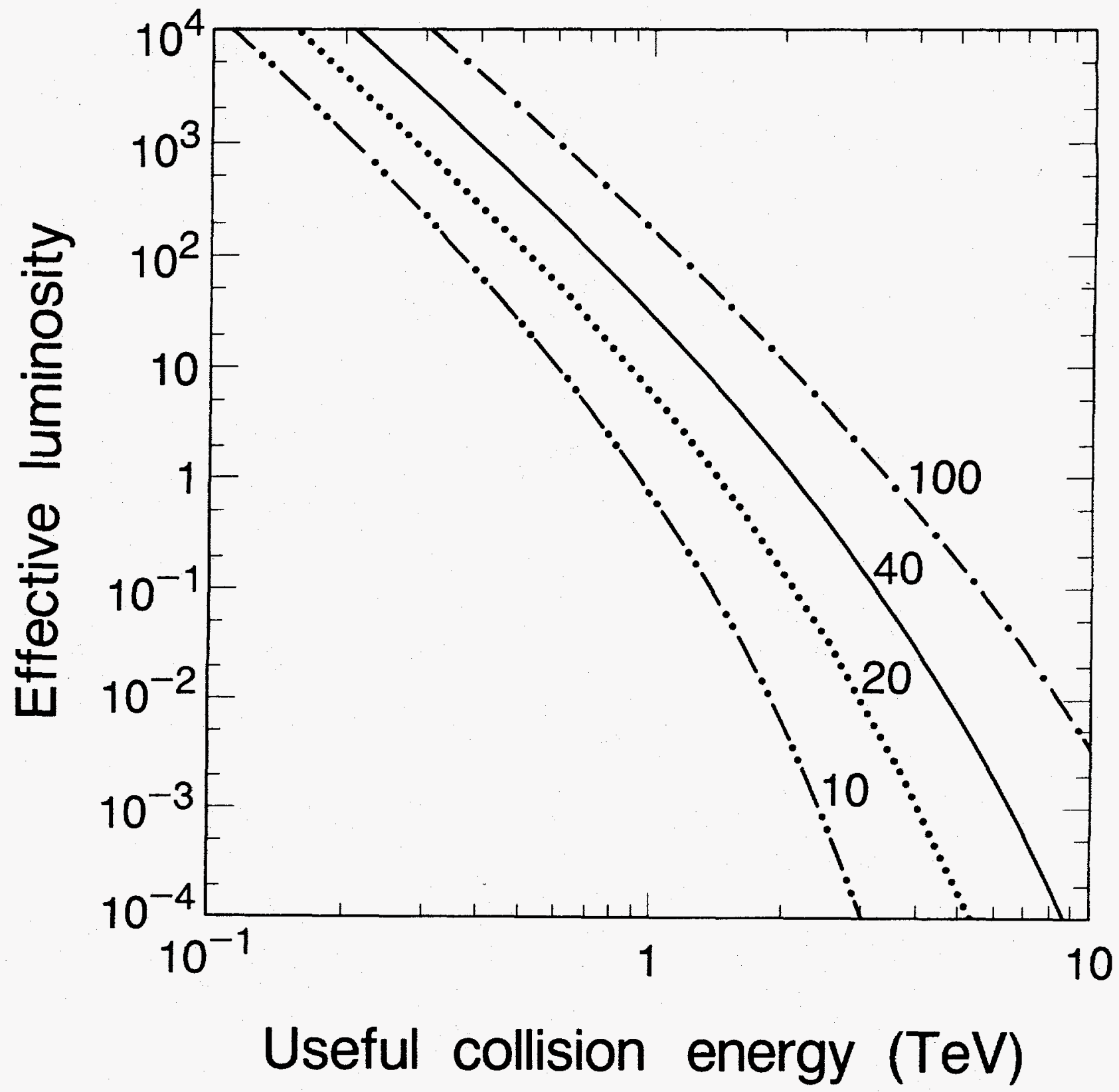

Figure 2-3. The effective luminosity (see text) for gluon-gluon interactions as a function of $2 \hat{E}$, the useful energy available in a gluon-gluon col1ision. The numbers on the curves are twice the proton beam energy in a collider. The solid curve corresponds to the SSC. 
equal beam energies, $p \bar{p}$ and $p p$ colliders are essentially equivalent. Considerable success has been registered at current energies with $p \bar{p}$ collisions which have the advantage that, at least at low beam intensities, only one ring of magnets is needed. Two of the three hadron colliders built to date are $p \bar{p}$ machines. Note that the luminosity of the pp machine (ISR, see Table I(a)

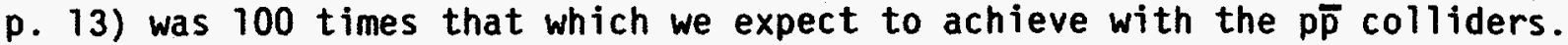
Note also, as was observed above, the luminosity required rises roughly as the square of the interaction energy being studied. For this reason, while future technological developments may make high luminosity $p \bar{p}$ colliders feasible, the current SSC design utilizes colliding proton beams, counter circulating in separate rings.

\subsection{DISCOVERY POTENTIAL OF A MULTI-TEV COLLIDER}

The need to probe mass scales of order $1 \mathrm{TeV}$ is discussed in Sect. 2.1. We must ensure that the SSC has sufficient energy and luminosity to carry out this search effectively. Thus, all of the theoretical options must be considered in order to ensure that the SSC is capable of exploring each and every one of them. This task was undertaken in detail by a number of researchers.*

\section{(1) Higgs Particle}

The simplest possibility is that there are no additional particles beyond those present in the standard model. As discussed in Sect. 2.1, this circumstance implies that either there will exist a well-defined scalar Higgs particle with mass considerably less than $1 \mathrm{TeV}$ or there will be some structure in the final state of $2 W^{\prime} s$ or $2 Z^{\prime}$ 's when the sum of the transverse energies of the W's or Z's is of order the Higgs particle mass. As this mass increases the cross section for such events will fall since the fraction of the total energy going into the subprocess is increased.

A Higgs particle of mass, say, $600 \mathrm{GeV}$ will decay predominantly into either a pair of $W$ or a pair of $Z$ particles. An experimental search will therefore consist of looking for structure in the $W$ or $Z$ pair production cross section. This is illustrated in Fig. 2-4. Enough events must be produced so that we can clearly see the peak and so be certain of a discovery.

It is not clear how efficiently one can detect $W$ or $Z$ particles. The most pessimistic scenario assumes that only the leptonic decay modes of the particles are observable. If this is true, then the observation of final states of

* Working Groups on Testing the Standard Model and Beyond the Standard Mode1, in Proc. 1982 DPF Summer Study on Elementary Particle Physics and Future Facilities, June 28-July 16, 1982, Snowmass, Colorado, eds. R.

Donaldson, R. Gustafson, and F. Paige, Division of Particles and Fields, American Physical Society, p. 1-49, 155-297.

E.J. Eichten, I. Hinchliffe, K.D. Lane, and C. Quigg, Reviews of Modern Physics 56,579 (1984).

Physics Section of Proc. 1984 DPF Summer Study on the Design and Utilization of the Superconducting Super Collider, June 23-Ju1y 11, 1984, Snowmass, Colorado, eds, R. Donaldson and J.G. Morfin, Division of Particles and Fields, American Physical Society, p. 1-303. 


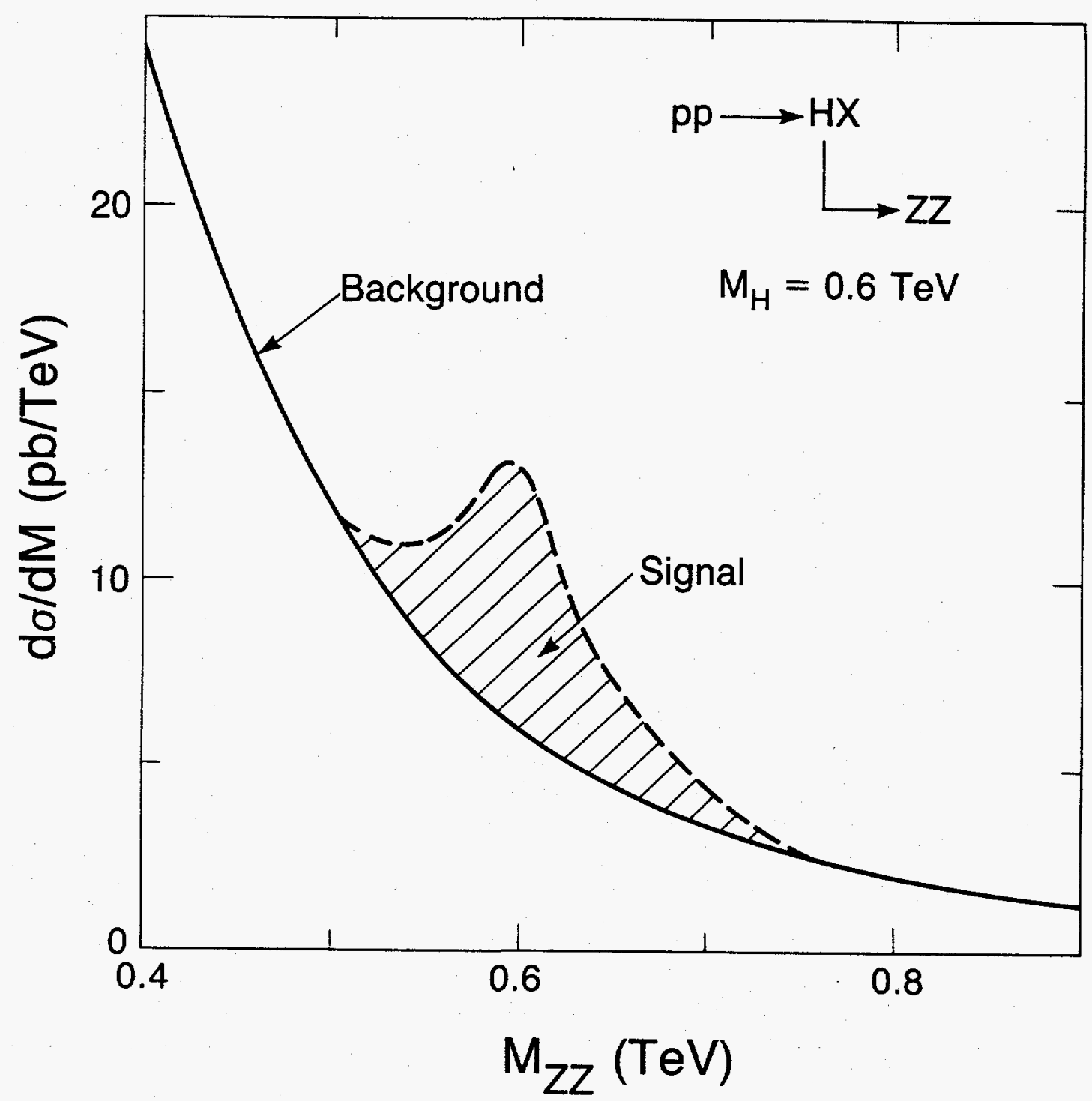

Figure 2-4. Cross section for production of two $z$ particles as a function of the invariant mass of the pair. The falling curve represents the "background" (from dynamically uncorrelated $Z$ pairs). The shaded "signal" represents production of a Higgs particle of mass $0.6 \mathrm{TeV}$ and its decay into a pair of $Z$ particles. 
four isolated electrons or muons originating from the decays $Z \rightarrow \mu^{+} \mu^{-}$and $Z \rightarrow e^{+} e^{-}$, will enable the Higgs to be detected. Requiring that a year of running produce 20 events in these channels implies that a beam energy of at least $10 \mathrm{TeV}$ and luminosity of $10^{33} \mathrm{~cm}^{-2} \mathrm{~s}^{-1}$ is necessary. Figure 2-5 illustrates the maximum Higgs mass that can be reached for different choices of the energy and luminosity using the same criterion. Notice that the luminosity is critical. A machine with a luminosity of $10^{31} \mathrm{~cm}^{-2} \mathrm{~s}^{-1}$ has only a very small window $\left(180 \mathrm{GeV}<M_{H}<200 \mathrm{GeV}\right)$ of discovery.

\section{(2) Supersymmetric Particles}

One other theoretical option involves the existence of supersymmetric particles. Again, as discussed above, their masses must be less than a few TeV if supersymmetry is to be relevant to the problems of electroweak mass generation. One supersymmetric particle of particular interest is the gluino, which is the spin $1 / 2$ partner of the gluon and will be produced in pairs and decay to give final states with jets (collimated groups of particles) and neutrinolike particles, e.g. photinos. In principle, photinos can be detected by absorption or scattering, but in fact are almost impossible to detect. Absorption or scattering can occur only with the real or virtual creation of the supersymmetric partner of some ordinary particle. Such particles are evidently very massive and therefore energetically impossible to create, or, if created virtually, make the probability of photino scattering exceedingly smal1. The escaping photinos will cause the detected jets to appear in violation of energy and/or momentum conservation.

There are serious backgrounds to gluino production, arising for example from the decay of top quarks where neutrinos are emitted. Consequently, a large number of events will be required to ensure a discovery. A detailed calculation indicates that we may need to produce 10,000 pairs to be sure of a discovery. Figure 2-6 shows the gluino mass range that can be searched in a year's running at various collider energies and luminosities. Again, it can be seen that high energy and luminosity are required in order to probe the TeV region. For example, a $20 \mathrm{TeV}$ collider operating at a luminosity of $10^{33} \mathrm{~cm}^{-2} \mathrm{~s}^{-1}$ will be able to find a gluino if its mass is less than 1.6 TeV, whereas if the luminosity is reduced by a factor of 100 , the mass must be less than $600 \mathrm{GeV}$ if a gluino is to be found. There is no appreciable difference in the production rate at proton-proton and proton-antiproton colliders with the same energy and luminosity.

(3) New Force (Gauge) Particle

The presence of additional massive force particles (similar to the $W$ and the $Z$ ) is required by theories in which parity becomes a good symmetry at high energy, or in some superstring theories. Present knowledge provides no estimate for the masses of these particles, if they exist, so it is important to be able to search as large a range as possible. Figure 2-7 shows the accessible masses of new $W$ particles if 1000 are to be produced in a year of running, a reasonable criterion if they are only observable via their decays into electrons or muons with branching ratios of a few percent.

This is one of the few cases where antiprotons may provide an advantage, since the initial state consists of a quark and an antiquark. It will be 


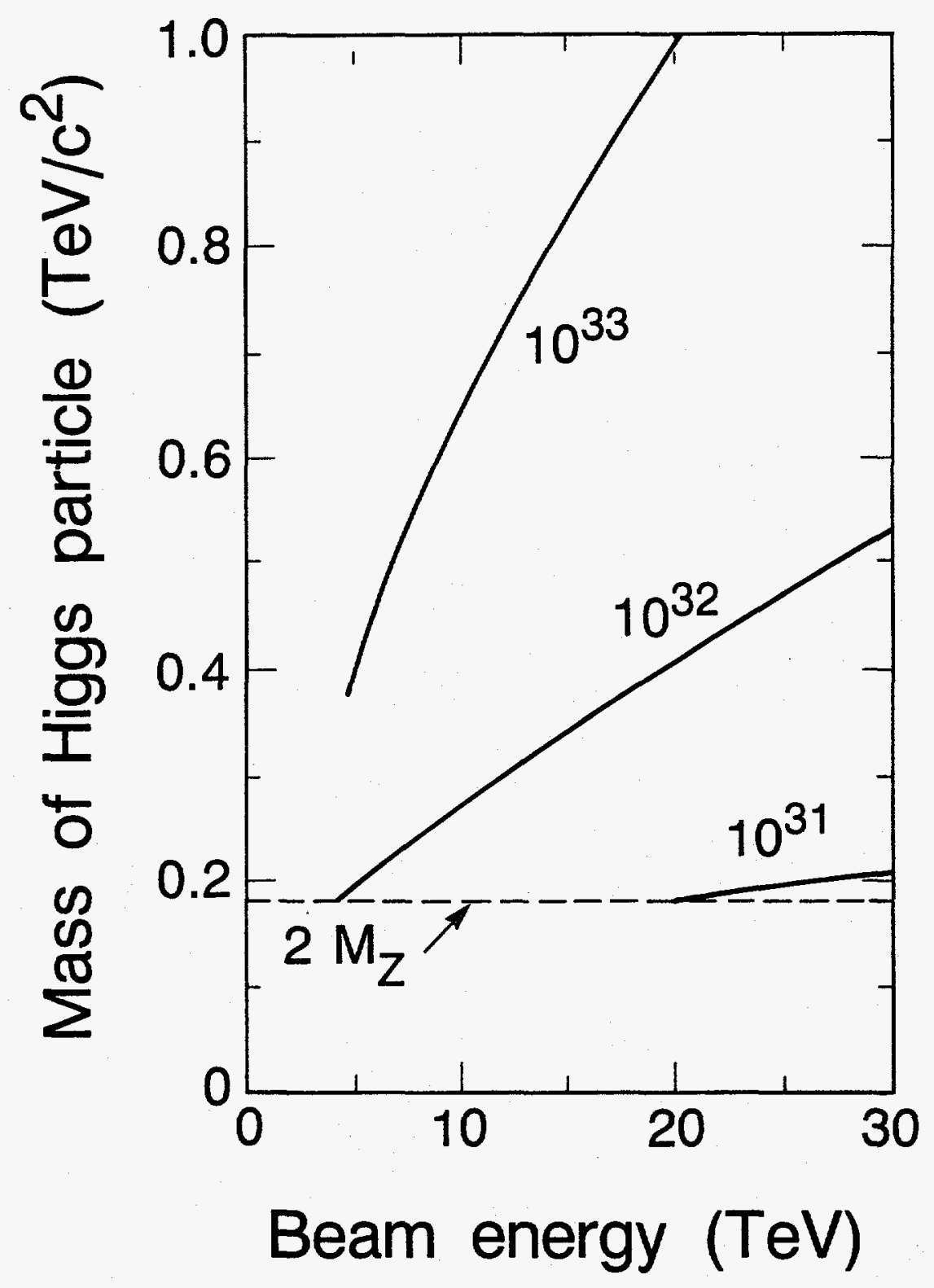

Figure 2-5. Upper and lower limits on the masses of Higgs particles that can be discovered with a hadron collider as a function of beam energy and luminosity. The effective lower limit of $2 M_{Z}$ is set by the difficulty of finding a decay signal if the Higgs particle is unable to decay into $Z$ pairs because its mass is less than $2 \mathrm{M}_{\mathrm{Z}}$. 


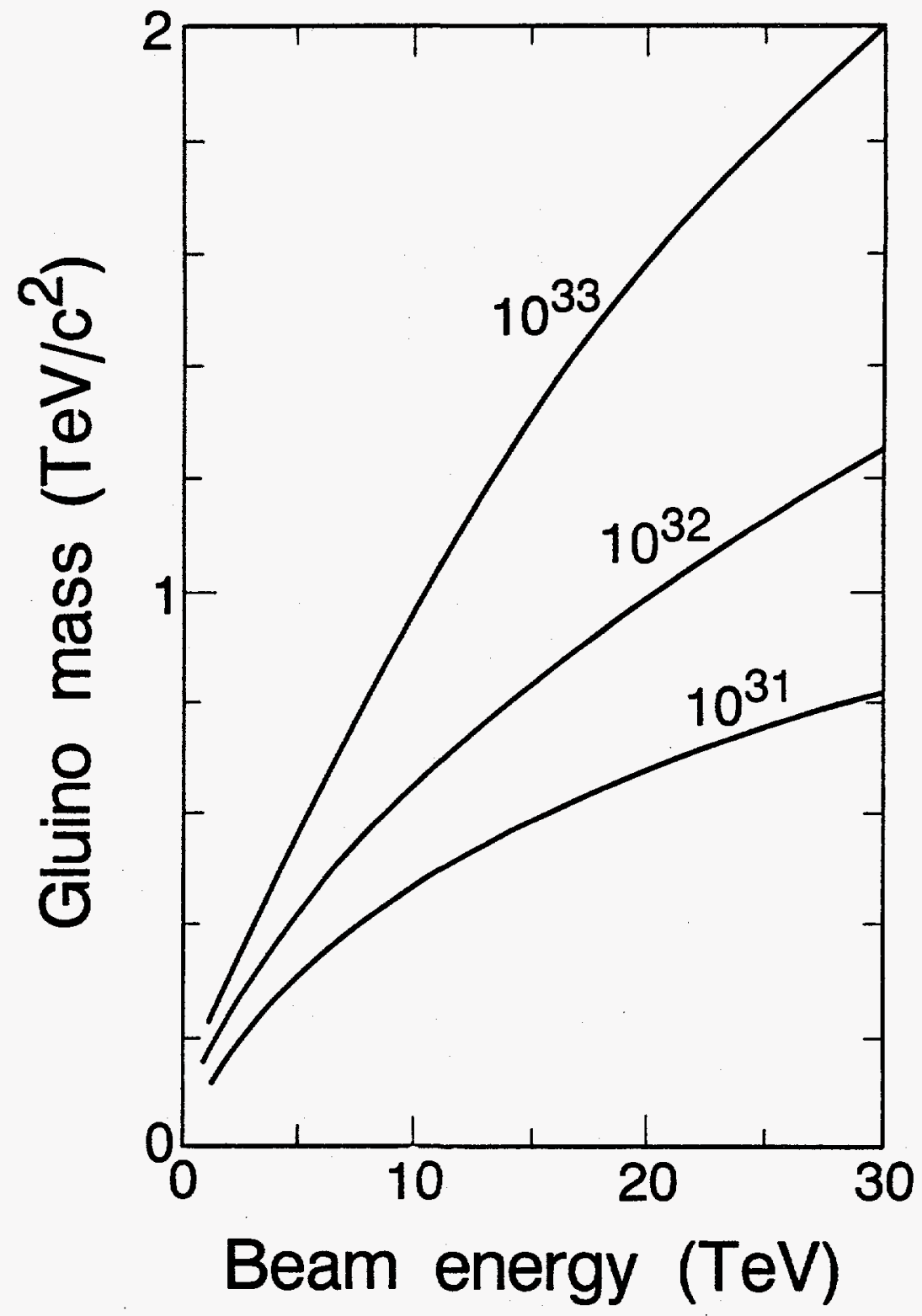

Figure 2-6. Discovery limits on masses for gluinos (supersymmetric partners of gluons) as a function of beam energy and luminosity for hadron colliders. 


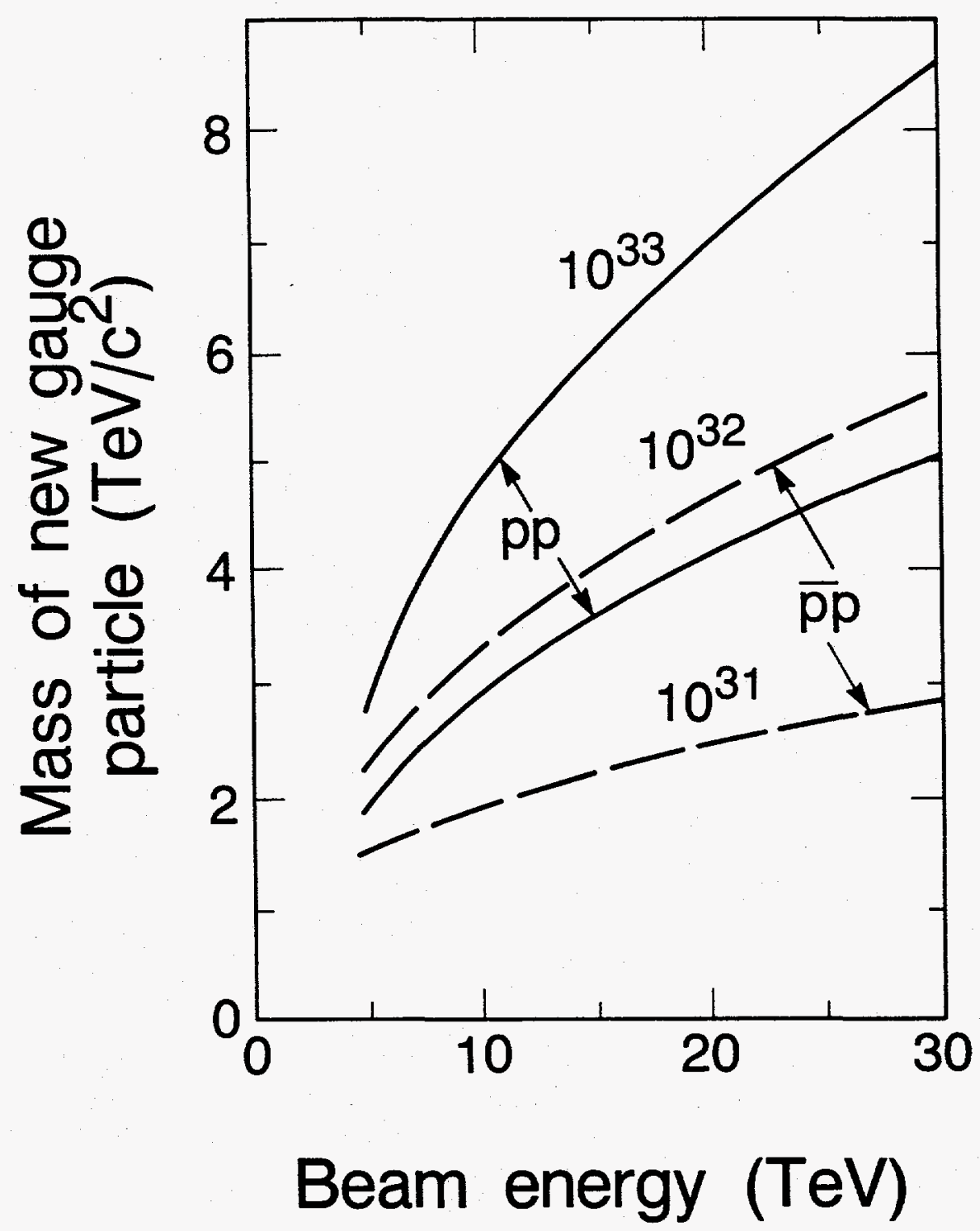

Figure 2-7. Discovery limits on masses of additional force (gauge) particles as a function of beam energy and luminosity for proton-proton colliders (solid lines) and proton-antiproton colliders (dashed lines). 
noted from the figure that this advantage is not present significantly in colliders with luminosity of $10^{32} \mathrm{~cm}^{-2} \mathrm{~s}^{-1}$, where the lines on the figure corresponding to proton-proton (solid) and proton-antiproton (dashed) collisions are not far apart. A proton-proton collider operating at $10^{33} \mathrm{~cm}^{-2} \mathrm{~s}^{-1}$ is certainly more effective that an proton-antiproton collider at one tenth of the luminosity.

\section{(4) Summary}

Figure 2-8 provides a summary of the capabilities of different colliders. This figure emphasizes that the power of the colliders increases dramatically as their energies and luminosities are increased. The increase in capability is almost independent of the particular search being conducted as can be seen by comparing the relative heights of the histograms on the figure. For example, a $20 \mathrm{TeV}$ collider operating at $10^{33} \mathrm{~cm}^{-2} \mathrm{~s}^{-1}$ (case 0 ) is sensitive to gluino masses 4 times larger than those which can be discovered at a $10 \mathrm{TeV}$ machine operating at $10^{31} \mathrm{~cm}^{-2} \mathrm{~s}^{-1}$ (case A). It is to be noticed that in one of the most critical areas, that of searching for a Higgs particle, colliders with luminosities of $10^{31} \mathrm{~cm}^{-2} \mathrm{~s}^{-1}$ (cases $A$ and $B$ ) are really inadequate. This is the one particle whose mass is limited by theory and only the $20 \mathrm{TeV}$ collider operating at $10^{33} \mathrm{~cm}^{-2} \mathrm{~s}^{-1}$ is capable of searching the entire range.

\subsection{RECAPITULATION}

The recent progress of particle physics has brought astounding results. A distinct level of matter below the proton has been identified. Three generations of that matter have been found. The basic forces between these particle have been identified. Moreover, the weak and electromagnetic forces have been united into a single theory which has passed every experimental test so far. The theory correctly predicted the approximate mass of the previously und iscovered charm quark and the precise masses of the $W$ and the $Z$. This theory is extraordinarily successful, but still is not complete. Undiscovered is the Higgs particle or some alternative to it that must be there. Unclear is the reason for the duplication of the generations of particles and the pattern of their masses. Are there still more quarks and leptons? Are there additional forces and new forms of matter beyond those we have observed so far? Are there additional levels of matter beneath the quarks and leptons? These are some of the challenges in particle physics that the SSC will address.

In the search for a deeper understanding of Nature, scientists probe with a variety of instruments. The particle physicist must employ devices of very large size and great complexity to study the fundamental constituent particles from which all matter is made. The construction of these tools, ever larger and more intricate as we probe deeper and deeper, calls upon an unparalleled diversity of scientific and technological skills. The challenges posed by instruments like the SSC and the scientific and engineering solutions to them have repercussions far beyond the research laboratory. Improved materials, transfer of techniques to unrelated fields, new know-how all produce wealth in its most basic sense, completely apart from purely scientific discoveries. The scientific discoveries themselves deepen and intensify our understanding of the universe in which we live. Consider the changes in our view of the 

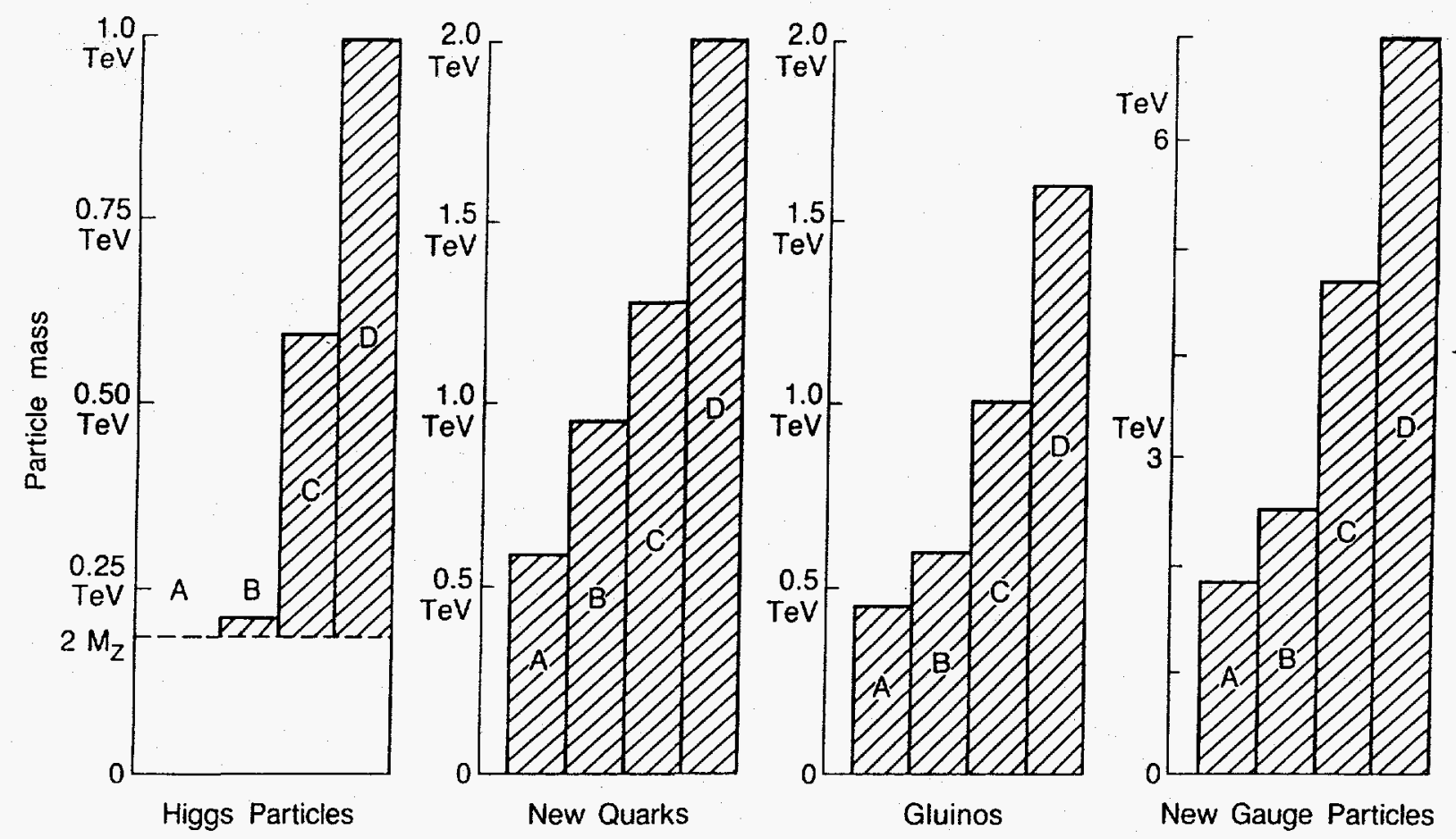

Figure 2-8. Histograms showing the discovery reach (maximum observable mass) of different hadron colliders for four possible types of new particles. The colliders are

\section{Designation}

A
B
C
D
Beam Energy (TeV)

10

20

10
Luminosity $\left(\mathrm{cm}^{-2} \mathrm{~s}^{-1}\right)$

$$
\begin{aligned}
& 10^{31} \\
& 10^{31} \\
& 10^{33} \\
& 10^{33}
\end{aligned}
$$

"Discovery" is defined as 20 events per year in the mode $H \rightarrow Z Z$, both $Z \rightarrow \ell^{+} \ell^{-}, 500$ events per year for new quarks, $10^{4}$ events per year for gluinos, and $10^{3}$ events per year for new gauge particles. 
world and its workings and our position in it that have occurred since the beginning of the present century. The SSC will contribute in a major way to a further development of that world view as we move towards the next century. 
Chapter 3

TECHNICAL BASIS FOR THE SSC

\subsection{INTRODUCTION}

The technical basis for the SSC is rooted in the experience and expertise gained in the construction and operation of large nuclear and high energy accelerator facilities throughout the world. The intricate and complex system of components of a large accelerator, described in Section 1.6, has been created and operated routinely in a number of locations, in ever increasing size. In principle, the extension of pre-Tevatron conventional accelerator technology to a machine of the capabilities of the SSC is straightforward, but in practice it is not.

The use of superconductivity on a large scale is the key that makes the SSC practicable. Superconductivity is the property of conduction of electricity with absolutely no resistance whatsoever, a property exhibited by some elements, compounds, and alloys at temperatures very near absolute zero. Discovered by a Dutch scientist, Kamerlingh Onnes in 1911, superconductivity was a laboratory curiosity until recent times, when a combination of scientific and technological developments led to its practical application.

The advantages of a ring of superconducting magnets are two-fold: (a) The magnetic field strength can be greatly increased over that attainable with conventional copper and iron magnets, increasing proportionately the maximum energy possible for a given sized ring; (b) the power consumption in resistive heat loss can be eliminated, with modest need of power elsewhere. Quantitatively, magnetic field strengths can be tripled with superconducting magnets, while power consumption in the magnet system can be reduced by factors of 10 or more. The conventional main ring of magnets at Fermilab dissipates approximately $130 \mathrm{MW}$ at full energy. In the SSC, an instrument with a particle energy capability of 40 times the Fermilab main ring ( $20 \mathrm{TeV}$ vs. $0.5 \mathrm{TeV}$ ), the losses in the magnets themselves are negligible, although there is roughly 30 MW needed for the refrigerator system to maintain the magnets in their cold superconducting state. Without superconductivity the SSC would need at least $4000 \mathrm{MW}$ for its main rings!

The development of a large synchrotron with superconducting bending and focusing magnets was pioneered by Fermilab. The successful operation of the Saver/Doubler ring in 1983 and the Tevatron Collider complex, already described in Sect. 1.6, provides a firm technological foundation and proof that a SSC-like accelerator is feasible.

The success at Fermilab is being exploited in Hamburg, West Germany, where the Hadron-Electron-Ring Anlage (HERA), a proton-electron collider, is currently under construction, as mentioned in Sect. 1.5. The maximum proton energy will be $0.8 \mathrm{TeV}$, comparable to the Tevatron, with 4.7 Tesla superconducting magnets patterned closely on the Fermilab design. This facility is expected to be operational in 1990. 
While the technical basis for the SSC is well established, it was realized early that R\&D effort was desirable to optimize the design for a cost-

effective and reliable facility. The following sections describe briefly some of the issues and the associated R\&D. A more detailed discussion of many of the subjects is given in the SSC Interim Report,* and also in the forthcoming Conceptual Design Report.

\subsection{MAGNET DEVELOPMENT}

Our understanding of superconducting accelerator magnets has matured in the last few years; the Tevatron is operating, HERA is under construction in Europe. Many model magnets have operated successfully and superconducting materials are much better understood. To produce a minimum cost SSC, the magnet-bore diameter must be reduced to the limits allowed by beam dynamics (see Sect. 3.4), and long magnets must be built using mass-production techniques. The R\&D efforts of the past two years have clarified the issues of field strength, field uniformity, predictability and reproductibility of performance, and have led to a clear choice of magnet type for the conceptual design of the SSC.

\section{(1) Magnetic Components}

Because reproducible, reliable, and long lasting bending magnets with good field characteristics are essential and are the single most costly technical component of the SSC, the magnet R\&D effort has been considerable.** Initially, parallel efforts were pursued to explore a number of alternative styles of magnet. The various ideas (two magnetically coupled beam tubes and coils in a single thermally insulating cryostat; an iron-dominated, low field design; an improved design based on the Tevatron magnets) werc explored to determine whether potential cost savings in construction and/or in operation of the accelerator were indeed realizable.

In the course of these efforts at the national laboratories (Brookhaven National Laboratory, Fermilab, Lawrence Berkeley Laboratory) and the Texas Accelerator Center, many short (1 meter) and long (4.5 meters and more) model magnels ::cre built and tested. Figure 3-1 shows a sampling of test results on the peak field for six $4.5 \mathrm{~m}$ bending (dipole) magnets built at Brookhaven National Laboratory and eight $1: 0 \mathrm{~m}$ dipoles from Lawrence Berkeley Laboratory. The data show that all the R\&D magnets repeatedly achieved peak magnetic fields in excess of 6 tesla at $4.2 \mathrm{~K}$ after only a few cycles of bringing the magnet up to full power. Some small variation from magnet to magnet can be seen as a consequence of different superconducting cables being used. The trend towards higher peak field with successive LBL models is a reflection of the improvement of the quality of the superconductor, as is discussed in Sect. 3.3 .

A significant feature of these results is the evidence for higher peak fields at lower operating temperatures. The BNL models achieved fields close

* SSC Central Design Group, "Interim Report," SSC-SR-1011 (June 1985). ** "Supercollider R\&D: The First Two Years", URA booklet (December 1985). 

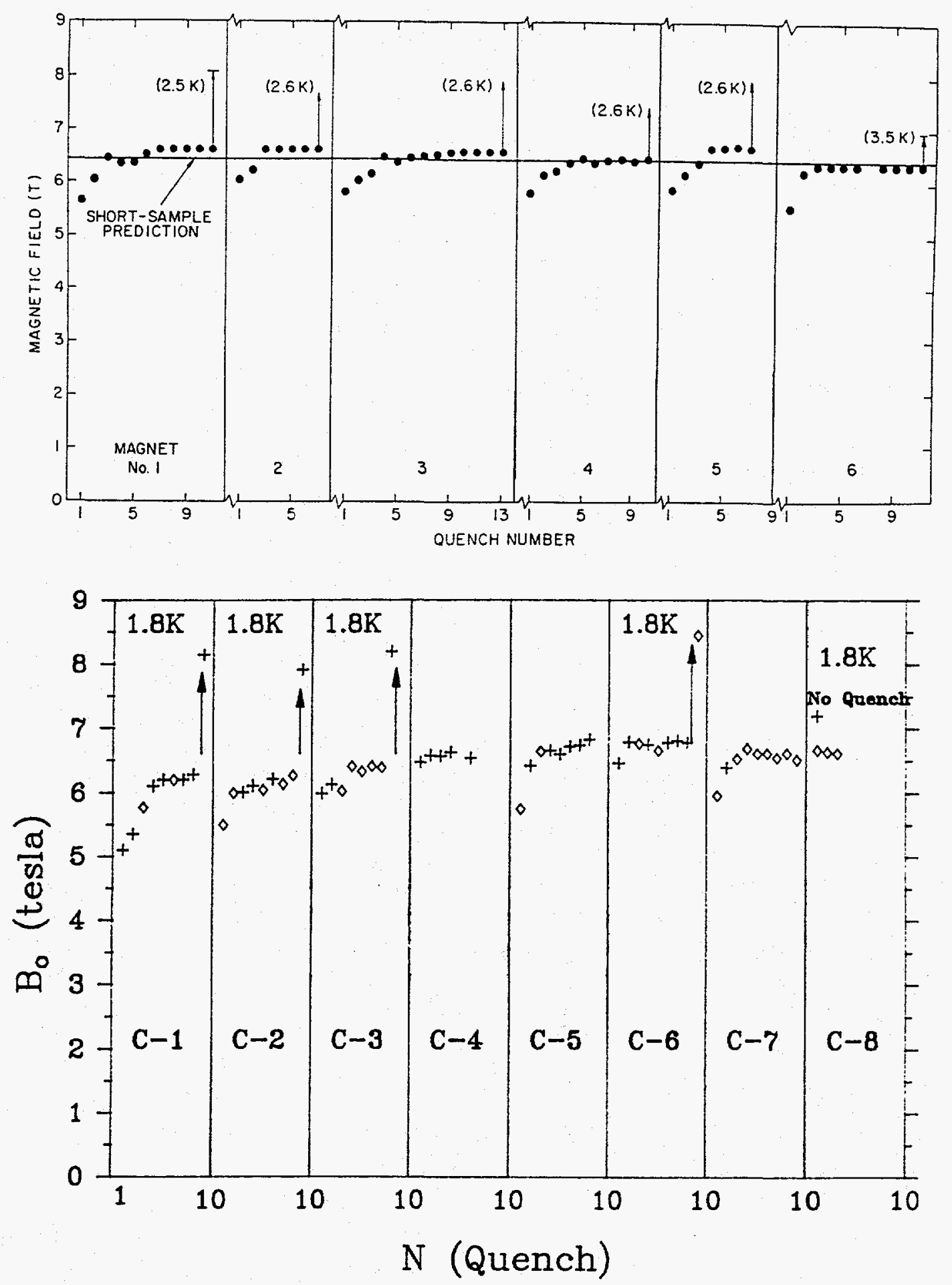

Figure 3-1. Peak magnetic fields attained at $4.2 \mathrm{~K}$ in model magnets upon successive slow rampings up in current. At top are data from six 4.5 meter "type D" model magnets made at Brookhaven National Laboratory. Below are the results for eight 1.0 meter "type $D$ " models built at Lawrence Berkeley Laboratory. 
to 8 tesla when operated at $2.5 \mathrm{~K}$ rather than $4.2 \mathrm{~K}$, while the LBL models exceeded 8 tesla at $1.8 \mathrm{~K}$. Lower operating temperatures would permit an increase in the maximum possible field and energy of the protons for the same sized ring and the same magnets, although such operation would require increased refrigeration capacity.

The uniformity of the field in the bending magnets is as important as the maximum attainable field strength. Small systematic departures from uniformity can be compensated by correction coils, but random departures because of inevitable small errors in fabrication cause nonuniformity of the field. that varies randomly from one magnet to the next. Such variations must be kept within acceptable limits to have a successful accelerator.

The departures from the ideal of a uniform field are described in terms of multipole coefficients $\left(a_{n}, b_{n}\right)$ which give the relative contributions of successive multipole fields (quadrupole, sextupole, etc.). A convenient unit of measurement of the multipole coefficients is $10^{-4} \mathrm{~B}_{0}$, that is, one part in 10,000 of the main dipole field. If multipole coefficients are kept to the order of magnitude of a few or less, in these units, the proton beams can be successfully guided through the confinement system without loss.

Data from a sample of six model magnets (type $D, 4 \mathrm{~cm}$ aperture, $4.5 \mathrm{~m}$ long, built at Brookhaven National Laboratory) are shown in Fig. 3-2. The particular design has certain relatively small systematic multipoles that need not concern us here (since they are easily corrected for). The important question is reproducibility, in other words, the random errors or deviations found in a set of actual magnets. On the basis of the details of the magnet fabrication process and its various tolerances, a number of models were developed to permit estimation of the random errors. Model parameters were adjusted to fit the measured random errors of the Tevatron and CBA magnets and then make predictions for the SSC magnets. The rms deviations of the various multipole coefficients expected from this analysis are shown by the open circles and triangles in Fig. 3-2. The actual rms deviations measured for the integral field in the six BNL models are given by the solid circles and triangles. For the lowest 12 coefficients the measured random errors are a 11 less than the predictions. For the highest order multipoles studied, the coefficients are zero within measurement errors.

Since the expectations of the models were the basis of the aperture studies described in Sect. 3.4, and the measurements indicate even smaller random errors, one can conclude that the manufacturing techniques are capable of producing many identical magnets of completely acceptable field quality. within tight tolerances.

The conclusions of the extensive R\&D effort on model magnets are that the magnet performance can be predicted reliably, that model SSC magnets achieve the necessary peak field strengths and have adequate field quality for accelerator operation, that the magnet fabrication techniques assure reproducibility from magnet to magnet, and that these techniques are ready to be transferred to industry. On the latter point, it should be noted that, although the six Brookhaven $4.5 \mathrm{~m}$ model magnets were produced as prototypes in the laboratory, the fabrication methods and tooling were designed with largescale industrial production in mind. 


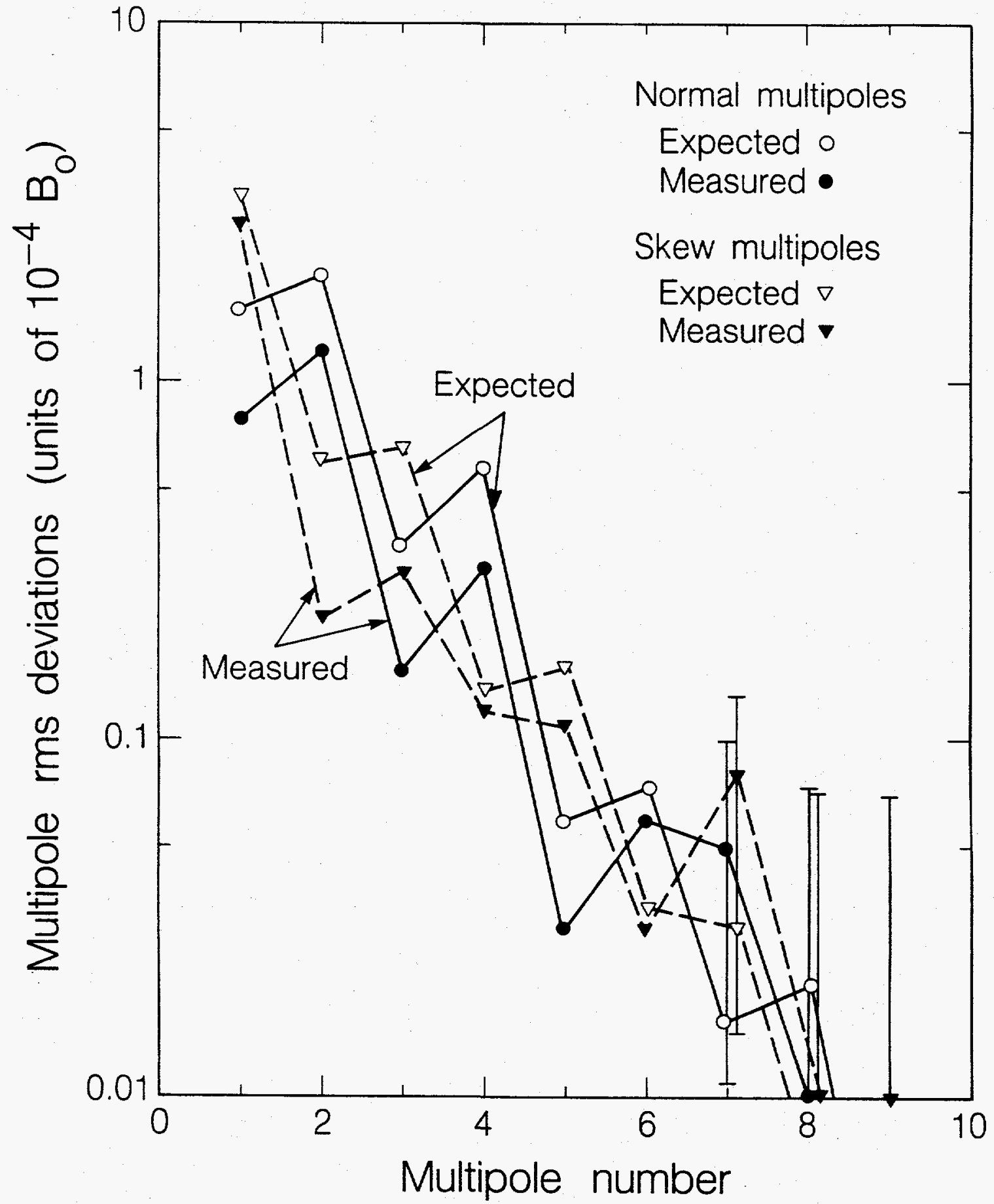

Figure 3-2. Expected and measured rms deviations of multipole coefficients $b_{n}$ and $a_{n}$ for the six BNL $4.5 \mathrm{~m}$ model dipole magnets of the style selected for the SSC. Within measurement accuracy, the actual multipoles are less than expected for all $\mathrm{n}$. 
Detailed consideration of model magnet performance, commissioning and operation of the accelerator, and cost comparisons of the magnets and magnetdependent systems led to the conclusion that a high-field, 1-in-l (one beam tube and coil assembly in one thermally insulating cyrostat), collared-coil, cold-iron design (called type D) was preferred as the basis for the SSC conceptual design and for further development into the actual SSC magnet. A perspective drawing of the so-called single-phase package or cold mass, the parts of the assembly kept at liquid helium temperature, is shown in Fig. 3-3. The diameter of the assembly is $26 \mathrm{~cm}(\sim 70 \mathrm{in.})$. The inner diameter of the inner layer coil is $4 \mathrm{~cm}\left(1.57^{\prime \prime}\right)$ and the inner diameter of the beam tube is $3.3 \mathrm{~cm}$. (1.3"). The design length of the magnet is approximately $17.3 \mathrm{~m}(57 \mathrm{ft})$. Roughly 7700 of these bending magnets and nearly 1800 focusing (quadrupole) magnets are needed for the two rings of the SSC collider. An important aspect of the continuing R\&D effort, now that a specific magnet configuration has been chosen, is the optimization of the design for ease of manufacture and long life.

(2) Cryostat Development

A superconducting magnet for an accelerator consists of a cold mass (beam tube, superconducting coils, stainless steel collars, and iron yoke), as shown in Fig. 3-3, installed in a cryostat or thermally insulating vessel. A cross section of the type $D$ magnet is shown in Fig. 3-4. The cold mass has passages in it for the flow of the liquid helium that maintains the superconducting coils near $4 \mathrm{~K}$. Nearby are liquid and gaseous helium return tubes. Outside an insulating jacket of superinsulation (aluminum-coated mylar layers) is the intermediate region of liquid nitrogen flow at $80 \mathrm{~K}$. Beyond that are thick layers of superinsulation surrounded by the outer vacuum-tight jacket at room temperature $(293 \mathrm{~K})$. The weight of each cold mass, roughly 8 tons, must be supported by posts that extend into the warm outside world, but prohibit as much as possible the leakage of heat into the cold regions.

The Tevatron cryostats (of a different design, because the iron in those magnets is on the outside) have a heat leakage of approximately $1.5 \mathrm{~W} / \mathrm{m}$ to the $4 \mathrm{~K}$ helium region. This heat must be conducted away from the coils by the circulating helium and so puts a load on the refrigeration system that must recool the heated helium for recirculation. While acceptable for the Tevatron, $1.5 \mathrm{~W} / \mathrm{m}$ in the SSC would be unacceptable. In the Reference Designs Study it was calculated that the cryostat heat leak to the $4 \mathrm{~K}$ and $20 \mathrm{~K}$ regions could be reduced to an acceptable $0.2-0.3 \mathrm{~W} / \mathrm{m}$.

The R\&D effort on cryostats has focused on the thermal and mechanical properties of the cyrostat, with the aims of reducing the heat leak per unit length and of studying the cryostat behavior during quenching. The careful positioning and overlapping of the layers of super-insulation and the design of posts with low thermal conduction are essential to reduction of the heat leak. A successful program at Fermilab has demonstrated a thorough understanding of the behavior of the cryostat during quenches and also shown that heat leaks of the order of $0.25 \mathrm{~W} / \mathrm{m}$ or less are achievable by careful design of the support posts and fabrication. A support post being readied for testing and the complete heat-leak cryostat test assembly are shown in Fig. 3-5. 


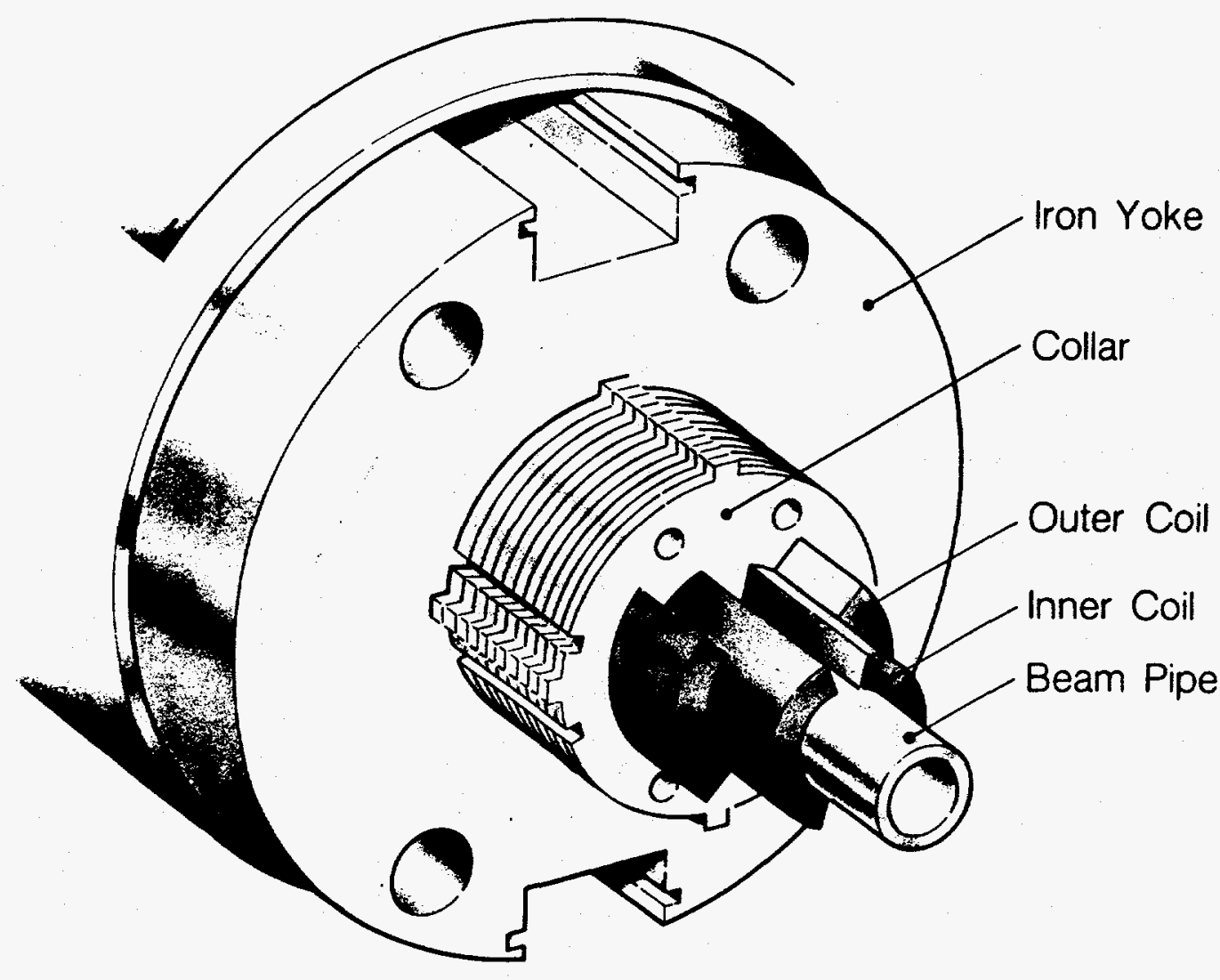

Figure 3-3. Perspective drawing of the inner (magnetic) components of the SSC dipole magnet. The iron yoke is $0.264 \mathrm{~m}(10.4 \mathrm{in.})$ in diameter. 


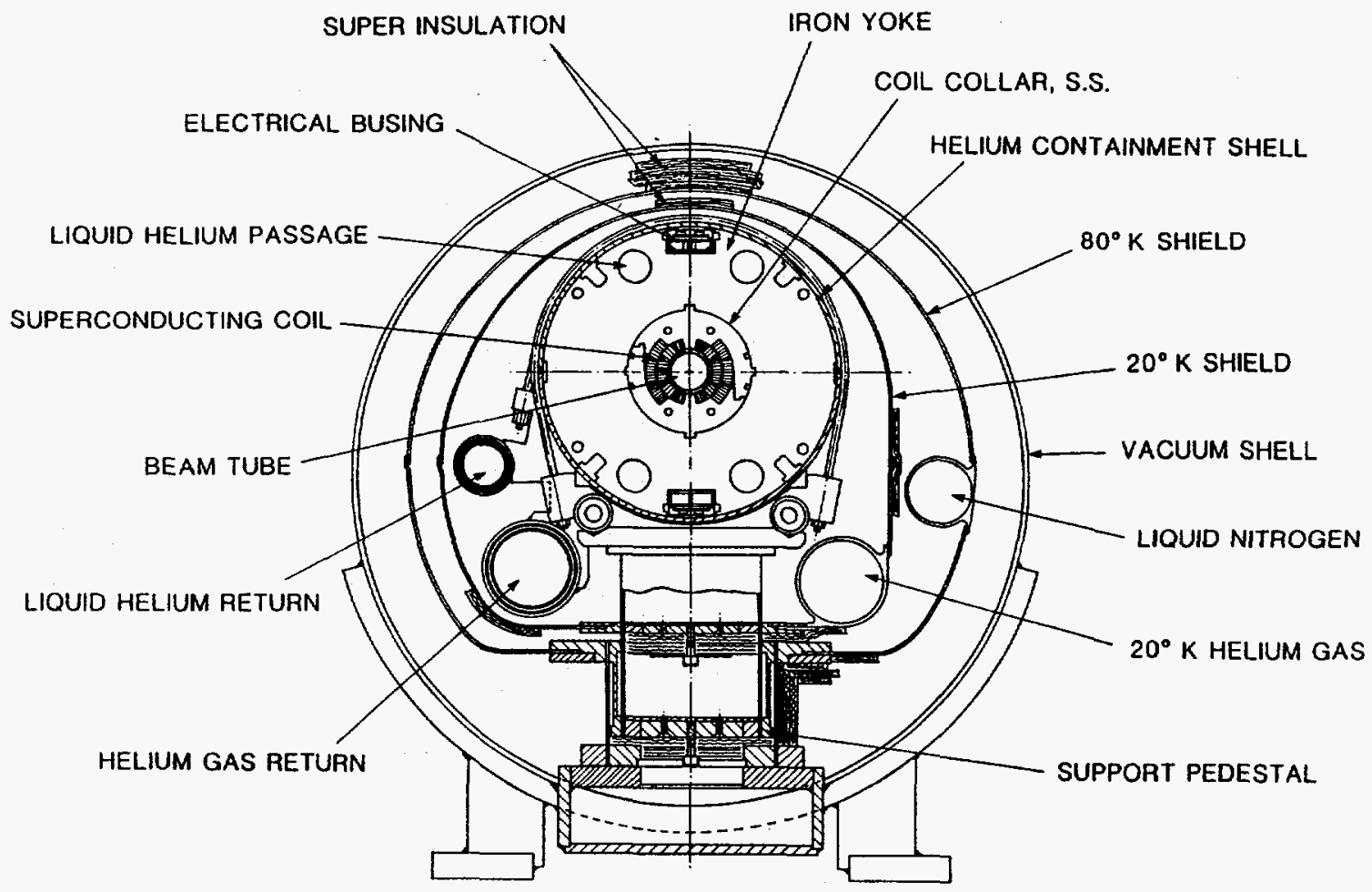

Figure 3-4. Cross section of the SSC dipole magnet assembly at a support post. The magnetic components are in a stainless steel helium containment vessel, surrounced by helium liquid and gas tubes, an insulating layer, a liquid nitrogen region, more insulation, and finally an outer vacuum shell of steel. The outer shell is approximately 0.61 meter (24 in.) in diameter. 


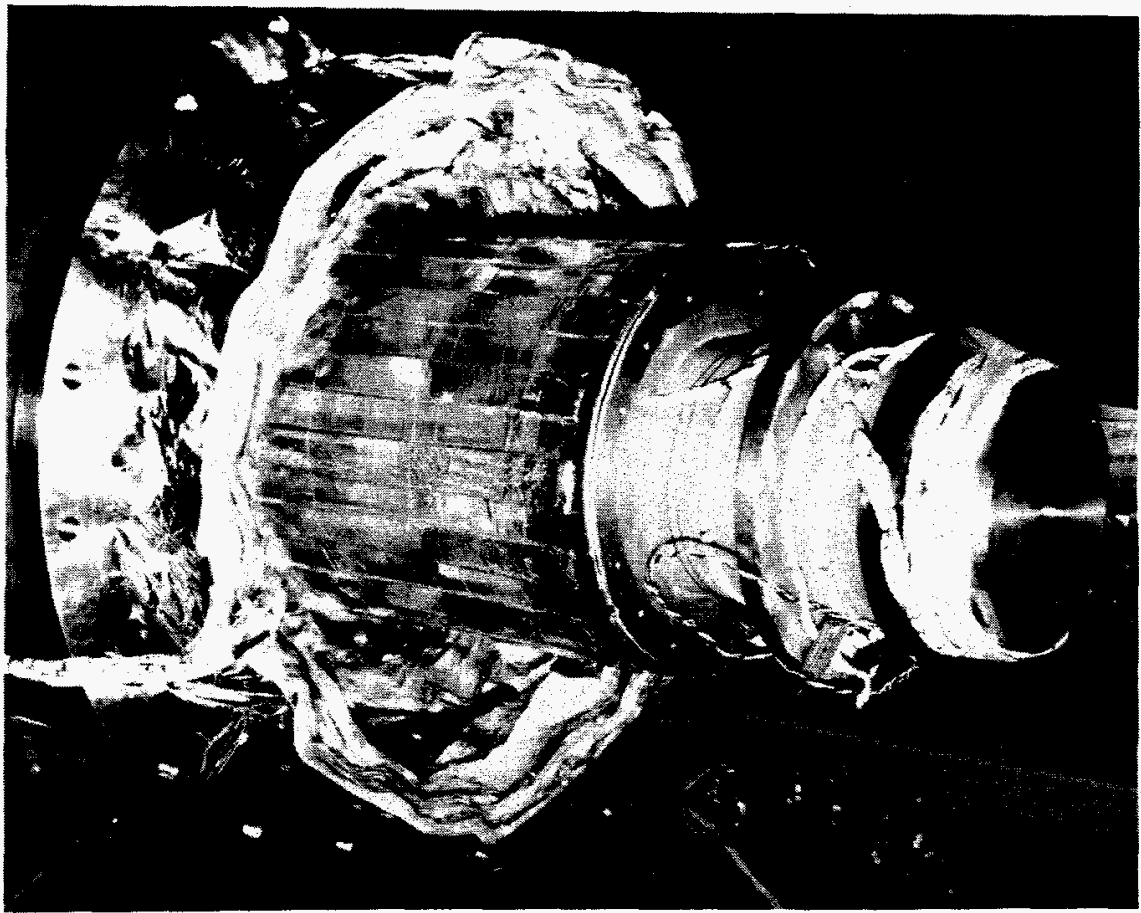

(a) Installation of a support post in the suspension heat leak measurement dewar. At this phase of the installation, the post is horizontal and the connection to the $20 \mathrm{~K}$ shield has been completed.

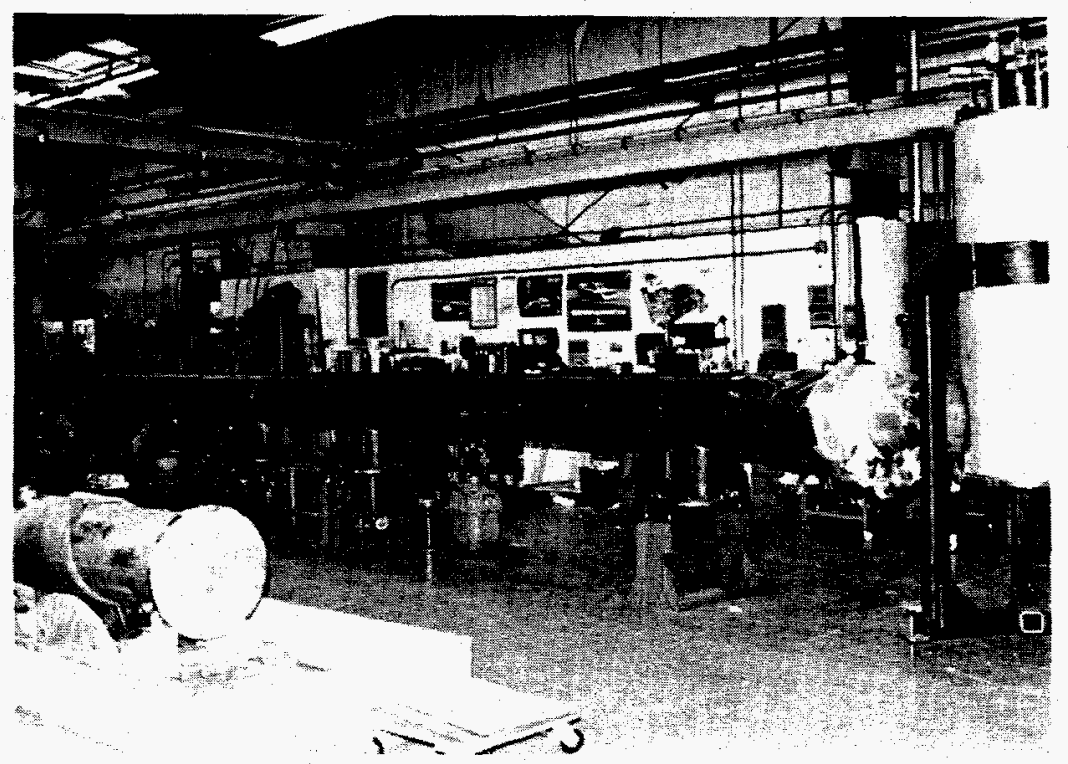

(b) Heat leak model cryostat during final preparation for connection of the center section to the end vessels.

Figure 3-5. 
(3) Further Development Efforts

The magnet R\&D program, having established the primary goal of selection of a preferred type for further development, is now focusing on the optimization of detailed magnetic and cryogenic features of the design and on the building of a series of full-length models. The model magnets are to be tested as a combined system, as well as individually, to test quench protection devices, the vacuum system, connections between magnets, and other aspects of the cryogenics. It is envisioned that the systems test will evolve into an accelerator systems test facility for the engineering development of other technical components. A separate life test will provide data on magnet lifetimes under extreme conditions, not ever anticipated in accelerator operation. The model magnet fabrication and test program is aimed at optimization and engineering development of the magnets, now that the most important questions have been answered.

\subsection{SUPERCONDUCTOR DEVELOPMENT}

The exploitation of superconductivity on a large scale in accelerators like the Tevatron depended up on long-term, productive interaction among the accelerator building community, the scientific experts on superconductivity, the commercial suppliers of the basic materials, and the wire and cable manufacturers. The employment of superconducting magnets in accelerators, except for special purposes, has been slow in maturing because of the novelty and the intrinsic difficulties with the physical and magnetic properties of the materials. For a number of technical reasons, superconducting alloys are preferred for the winding of coils for magnets. Two alloys in particular were singled out for development, niobium-titanium ( $\mathrm{NbTi}$ ) in a $46 \% / 54 \%$ ratio, and niobium-tin $\left(\mathrm{Nb}_{3} \mathrm{Sn}\right)$. The $\mathrm{Nb}_{3} \mathrm{Sn}$ compound has the advantages of higher maximum magnetic field at a given temperature and higher current carrying capacity at high field (and so requiring less superconductor for a given field), but is a brittle material, difficult to fabricate into wire and flexible cable. While R\&D continues on $\mathrm{Nb}_{3} \mathrm{Sn}$ and other materials, the superior mechanical properties of NbTi have made it the center of development.

The superconducting state of a sample in a magnetic field can be destroyed in a number of ways, one of which is a change of the magnetic field with time. To enhance stability of the superconducting state against the inevitable changes of the magnetic fields of an accelerator, it was found necessary to develop multi-filament strand. Such strand typically consists of thousands of NbTi filaments embedded in a copper matrix, with a ratio of one or two parts by weight of copper to one of superconductor. An enlargement of the cross section of a representative strand is shown in Fig. 3-6.

A figure of merit of superconducting strand is the maximum possible current density $J_{C}$ (in amperes per square millimeter, or $A / \mathrm{mm}^{2}$ ) of the superconductor itself at $4.2 \mathrm{~K}$ and in a magnetic field of 5 tesla. The higher is $J_{c}$, the less is the amount of superconducting material needed for a given peak magnetic field. Since superconductor is expensive, there is a premium on increasing the current-carrying capacity of the NbTi alloy, provided the cost of the improvement does not cancel the savings from use of less superconductor. At the time of construction of the magnets for the Tevatron (1980-83), commercial strand had $J_{C}$ in the range of $1800-1900 \mathrm{~A} / \mathrm{mm}^{2}$. In the Reference 


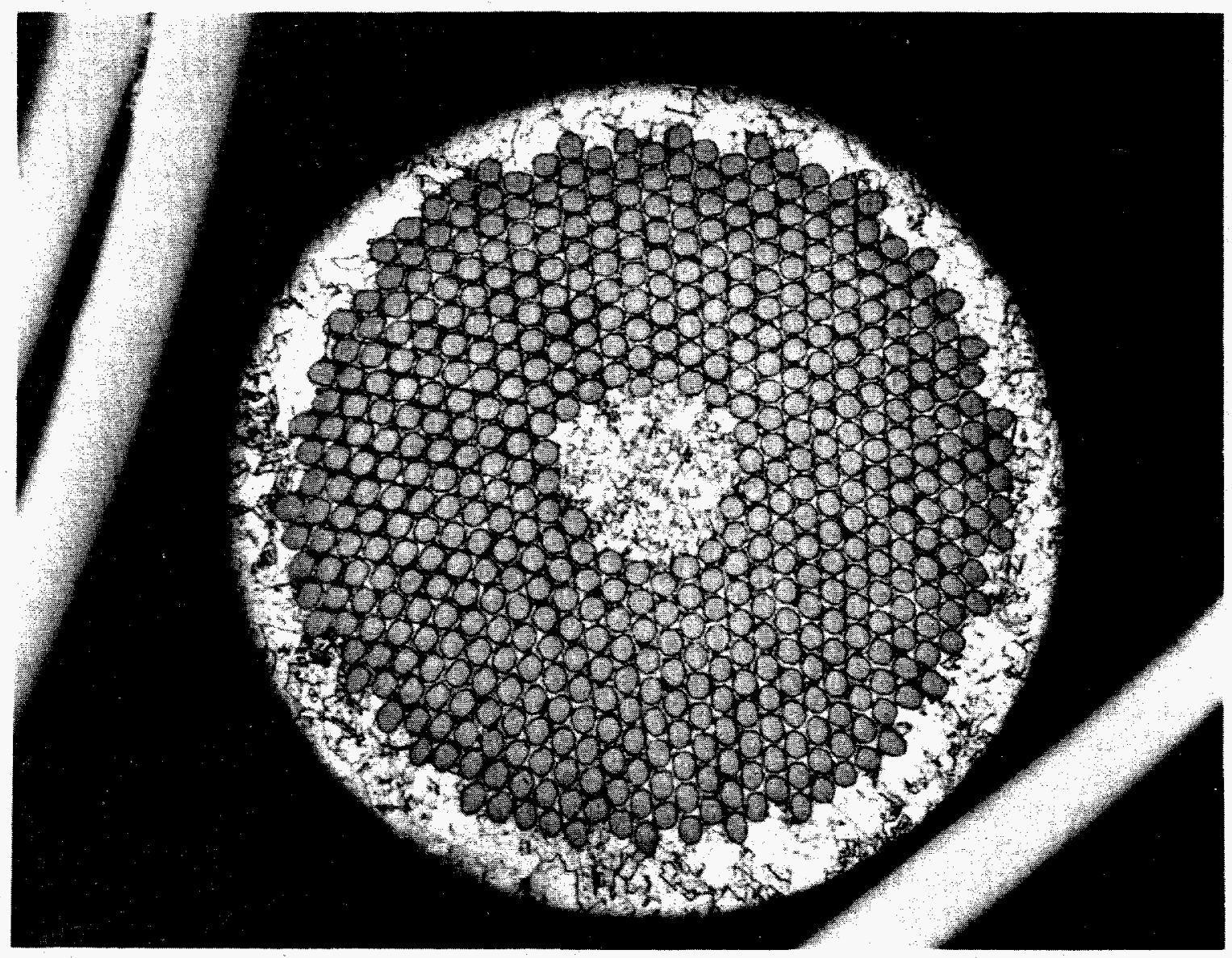

Figure 3-6. Microphotograph of multi-filamentary superconducting strand for SSC magnets. Overall diameter is $0.81 \mathrm{~mm}$. The filament diameter is $20 \mu \mathrm{m}$, and the copper-to-superconductor ratio is $1.3: 1$. (Courtesy of Intermagnetics General Corporation) 
Designs Study (Spring of 1984) it was assumed that improvements in $J_{c}$ would occur without significant cost increases to values of at least $2400 \mathrm{~A} / \mathrm{mm}^{2}$. This value was taken as the nominal SSC specification.

(1) Improvements in $\mathrm{J}_{\mathrm{C}}$

In the past two years a DOE-funded R\&D program aimed at superconductor improvements has been carried out jointly by national laboratories (Brookhaven National Laboratory and Lawrence Berkeley Laboratory), universities (University of Wisconsin), and industry. The program has been remarkably successful. Crucial areas for improvement were identified--reduction of the small ( $\pm 5 \%$ ) fine-scale fluctuations in the homogeneity of the NbTi alloy, greater care in the heat treatment and drawing processes, coating the NbTi rods with a thin barrier layer of $\mathrm{Nb}$--then developed in the laboratory, largely at Wisconsin under the direction of D.C. Labalestier, and successfully transferred to industry.

A measure of the dramatic success of the program is shown in Fig. 3-7. The joint effort has produced a $50 \%$ increase in $\mathrm{J}_{\mathrm{C}}$, from the 1800-1900 $A / \mathrm{mm}^{2}$ of the Tevatron strand to $2500-3000 \mathrm{~A} / \mathrm{mm}^{2}$, in the past two or three years. The nominal RDS value of $2400 \mathrm{~A} / \mathrm{mm}^{2}$ has been surpassed. Present SSC magnet design is based on $2750 \mathrm{~A} / \mathrm{mm}^{2}$.

\section{(2) Finer Filaments and Cabling}

The SSC magnets must have a spatially very uniform field over a range of field strengths from 5-10\% of full field to full field. Eddy currents that do not decay are induced in superconducting filaments when the field is changed. These persistent currents cause a distortion in the field that, for a given conductor, is roughly proportional to filament size. Such field distortions, which are most important at low field (injection), must be corrected if they are above some minimum level. While it is possible to put small coils along the length of the magnet on the outside of the beam tube to provide the correction, it is desirable to work towards finer filaments in the strand to make such coils unnecessary. Filaments sizes of 15-25 $\mu \mathrm{m}$ are now typical of regular strand; with it, persistent currents would be significant at injection. The R\&D program for improvement of the superconductor therefore has as a goal the production of strand with fine filaments (as small as 2 or $3 \mu \mathrm{m}$ in diameter), as well as higher maximum current-carrying capacity.

Proper processing and quality control, as well as use of the thin barrier of niobium, has led to the production by several manufacturers of samples with fine filaments ( 2 to $4 \mathrm{\mu m}$ in diameter) and excellent current-carrying capabilities. A U.S. company has achieved a sample strand with 3 um diameter filaments and $J_{C}$ of $3000 \mathrm{~A} / \mathrm{mm}^{2}$. [See Fig. 3-7].

For use in the fabrication of magnets, strands must be wound into a cable of precisely controlled dimensions. The cable, typically $0.1-0.2 \mathrm{~cm}$ thick and $1 \mathrm{~cm}$ wide, is made up of 20 to 40 strands, wound in a spiral pattern, as shown in top half of Fig. 3-8. An enlarged cross section of a 23-strand cable for the model SSC magnets is shown in the bottom half of the figure.

R\&D on cabling techniques has occurred at Lawrence Berkeley Laboratory as part of the SSC effort, with results transferred to industry. Production speeds of the order of $10 \mathrm{ft} / \mathrm{min}$ are presently attainable in the laboratory 


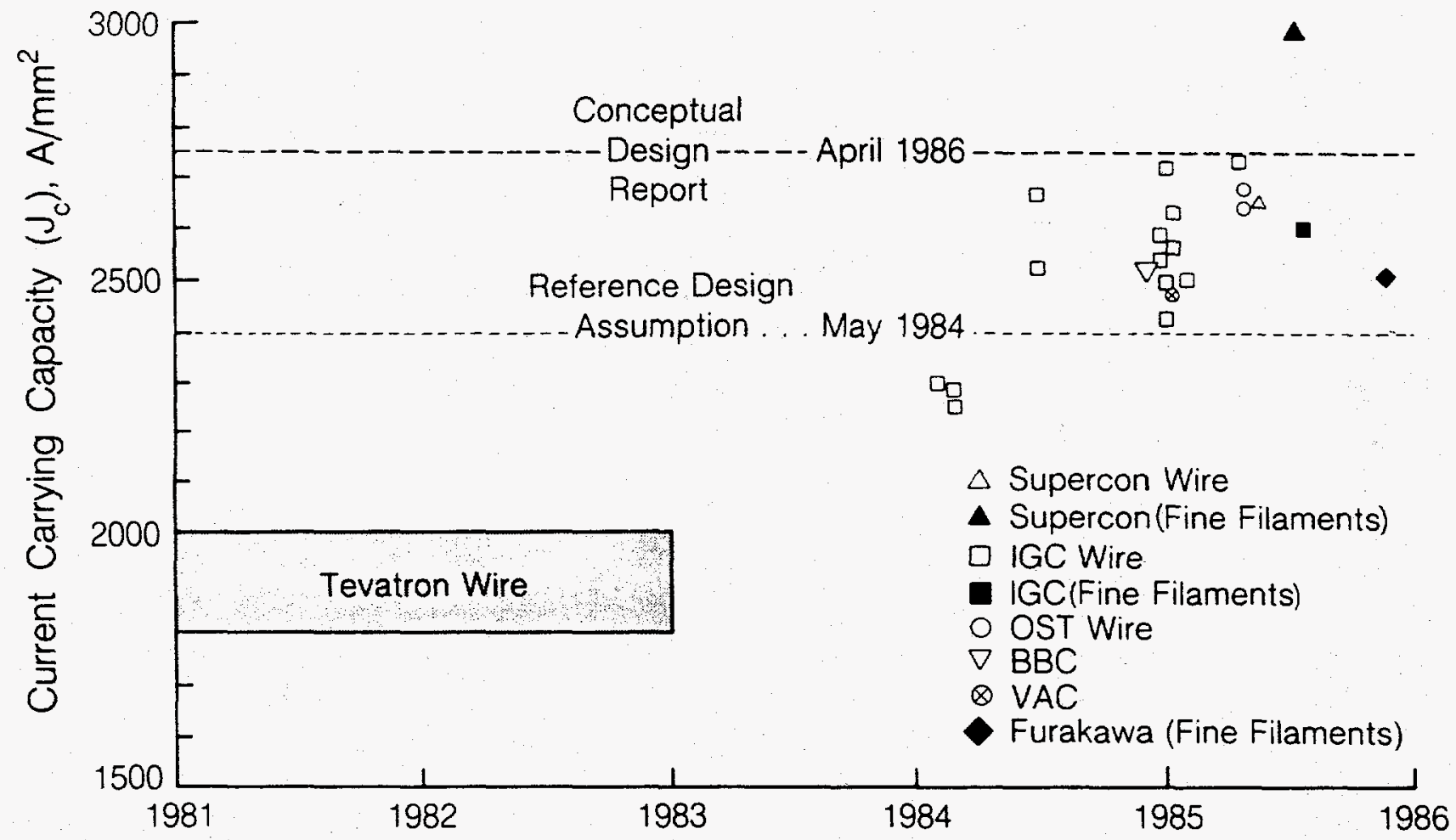

Figure 3-7. Achievable critical current density $J_{c}$ of superconducting $\mathrm{NbTi}$ strand (in $\mathrm{A} / \mathrm{mm}^{2}$ at a temperature of $4.2 \mathrm{~K}$ and a magnetic field of 5 tes la) as a function of time. The lower rectangle represents the spectrum of values for the strand used in Tevatron magnets. The improvement in the past two years is striking. 

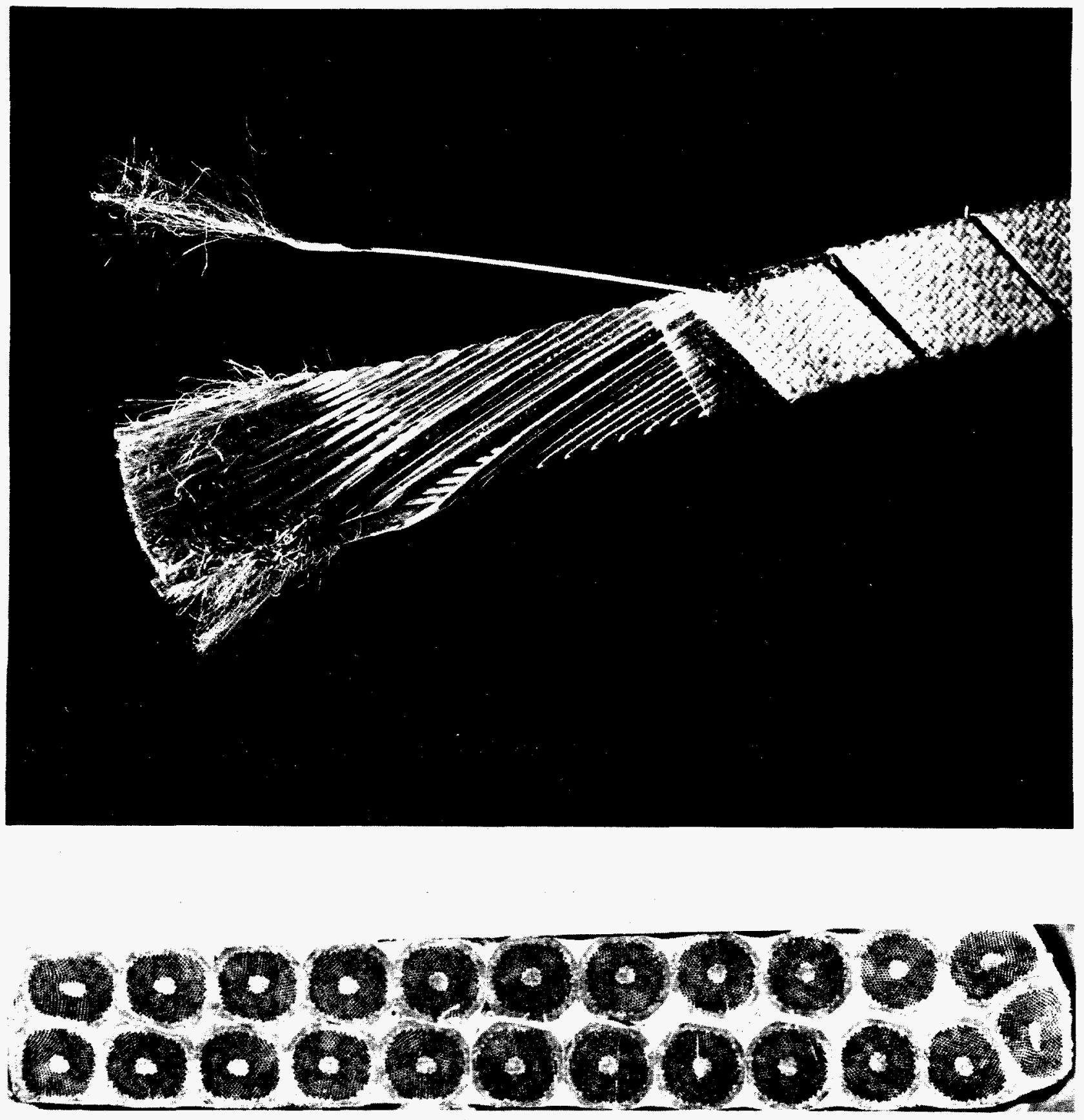

Figure 3-8. Enlargements of SSC superconducting cable. (Upper) Spiral wound cable of 30 strands, approximately $1 \mathrm{~cm}$ wide, wrapped with insulating tape. The ends of the strands have been etched in acid to remove the copper and reveal the NbTi filaments. (Lower) Cross sectional view of a 23 strand cable (enlarged approximately 16 times). The "keystone" shape conforms to the arch-like configuration of the cable in the dipole coils. 
and in industry. Continuous lengths of cable up to $5000 \mathrm{ft}$ are possible. Problems associated with winding cable having 30 or more strands by industry have been solved by mutual efforts.

The fine filaments strand presents a cabling problem. Practical limitations of how many NbTi rods can be put in a copper matrix and drawn down leads to very small strand (a few tenths of a millimeter in diameter). Even then there may be 4000 filaments inside. To make a cable of the desired dimensions, a two-step cabling process has been developed. An initial seven-strand cable of the fine filament wires with a copper core becomes the "strand" for a 23-element cable of the final SSC dimensions. The cable developments in the laboratory are transferred regularly to the industrial cabling firms by means of workshops.

Remarkable progress in raising the maximum current-carrying capacity of the strand and in creating strand with fine filaments has occurred in the past few years through the cooperative efforts of government, industry and the universities. This progress benefits the SSC directly in lower costs and greater simplicity. The increased current-carrying capacity also benefits other users of superconducting wire and cable, for example, the manufacturers of NMR medical imaging devices with superconducting magnets.

\subsection{BEAM DYNAMICS AND APERTURE}

Successful operation of the SSC depends on the detailed understanding of the motion of thousands of bunches of protons accelerated in the cascaded accelerators of the injector and then accelerated and stored in the collider rings. The protons in the bunches make small excursions transversely and back and forth from the ideal position (in orbit and phase). Some of these deviations would be present in a perfect machine; others are induced by various initial conditions, perturbations and imperfections. If the departures from the ideal position are too great, the beam is lost.

The detailed study of beam motions is the domain of accelerator physics. A central issue is the definition of the "aperture", the cross-sectional area within which the particle motion is stable. If the available area is large enough, the accelerator will operate without rapid loss of beam. If it is too small (relative to, say, the needs of the beam emerging from the injector). the beam will be unstable and will be lost by striking the walls of its vacuum chamber. Clearly, one wants a comfortably large aperture. This can be achieved in many ways--injecting a very high quality beam needing a small aperture, or having a large region of very uniform magnetic field transverse to the beam, etc. Such solutions cost money. Injection of a very high quality beam presumes an elaborate and expensive injector complex. Larger diameter magnets mean more cost in materials, more stored energy, more elaborate quench protection and cryogenic capabilities, etc. The aperture issue is thus a compromise. One wishes the smallest possible physical aperture (inside dimension of the evacuated beam pipe), consistent with adequate space for stable motion of the particles in the beam (dynamic aperture). Relevant are the random imperfections in the magnets, as well as the systematic departure of the magnetic field inside the beam pipe from its ideal form because of limitations in magnet design. 
Detailed analytic and numerical methods have been used to study beam dynamics in the SSC and to determine appropriate physical dimensions for the beam tube under realistic assumptions about the magnet quality and reproducibility. Experiments have been undertaken at the Tevatron and at SPEAR to supplement and verify the numerical calculations. A sample of the results from one such experiment is shown in Fig. 3-9. The accelerator conditions were adjusted to enhance a particular type of beam motion having a three-fold symmetry in transverse velocity-position space. The beam was then displaced and its subsequent motion followed. The data show that the motion possesses the expected three-fold symmetry and is consistent with the predictions of theory.

The conclusion* of the aperture studies and associated cost comparisons is that a beam tube within a coil of approximately $4 \mathrm{~cm}$ (1.5 inches) inside diameter is adequate from the point of view of beam dynamics. The actual envelope of expected motion of the protons is approximately half as large. For comparison, the Fermilab Tevatron and the HERA magnets have an inner coil diameter of $7.5 \mathrm{~cm}$.

\subsection{SYNCHROTRON RADIATION AND VACUUM}

A charged particle moving at high speeds and bending in a magnetic field emits electromagnetic radiation, called synchrotron radiation because it was first observed in electron synchrotrons. The energy radiated in each revolution must be replaced by the radio-frequency accelerating system. In electron accelerators, the synchrotron radiation loss is significant and is the ultimate factor that makes circular electron accelerators beyond a certain energy uneconomical (roughly $100 \mathrm{GeV}$ beams, the ultimate energy of LEP). For proton accelerators, the situation is much less severe--for fixed radius of ring, the effect is proportional to $\left(E / \mathrm{mc}^{2}\right)^{4}$, where $E$ is the particle energy and $\mathrm{mc}^{2}$ is its rest energy. For the initially $50 \mathrm{GeV}$ electrons in LEP, the ratio is $E / \mathrm{mc}^{2}=10^{5}$. Because of their much larger mass, the $20 \mathrm{TeV}$ protons of the SSC give a ratio only $1 / 5$ as large, even though their energies are 400 times larger. In fact, the SSC will be the first proton accelerator in which the synchrotron radiation is at all significant.

Making up the radiated energy is no problem for the RF system, but the radiation does have an impact on the cyrogenic system and the vacuum. A $70 \mathrm{~mA}$ beam of $20 \mathrm{TeV}$ protons circulating in the SSC will radiate of the order of $9 \mathrm{~kW}$ in synchrotron radiation. The power (approximately $0.13 \mathrm{~W} / \mathrm{m}$ ) is dumped directly onto the wall of the cold beam tube. The helium flow must carry this heat away from the $4 \mathrm{~K}$ region, placing another burden on the refrigeration system. A total of $18 \mathrm{~kW}$ from the two beams necessitates about $10 \mathrm{MW}$ of refrigerator capacity, roughly two thirds of the total cyrogenic load at $4 \mathrm{~K}$.

In addition to the added heat load, the synchrotron radiation, whose broad spectrum consists mainly of soft $x$-rays in the 100-300 eV range, causes gas molecules $\left(\mathrm{H}_{2}, \mathrm{CO}, \mathrm{CO}_{2}\right)$ present in the metal of the beam tube to be ejected into the vacuum. If sufficient gas is knocked out, the quality of the vacuum

* Aperture Task Force, "SSC Aperture Estimate for Cost Comparisons", SSC-SR-1013 (August 1985) 


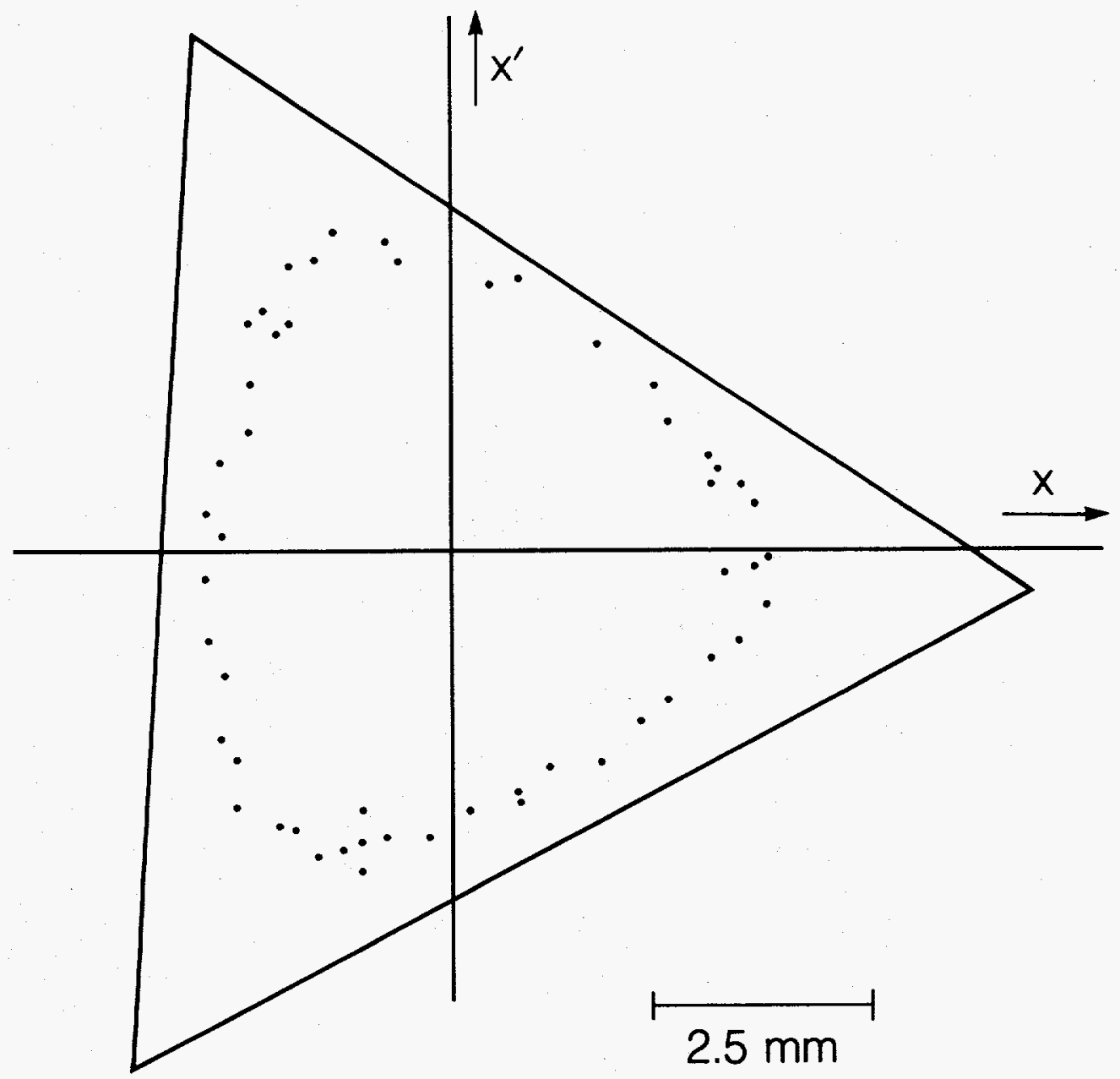

Figure 3-9. Horizontal position-velocity $\left(x, x^{\prime}\right)$ diagram for displaced bunch motion in a Tevatron experiment. The dots represent the "positions" of the bunch on successive revolutions past the detector. The triangle shows the expected domain of stable operation for the conditions of the experiment. 
can be affected and the lifetime of the stored beam reduced because of collisions with the gas molecules.

This photon-induced desorption of gases from metallic surfaces at liquid helium temperatures has previously been studied very little. It presented a potential expense of unknown magnitude and complexity, if severe. The solution in electron synchrotrons, where the phenomenon is severe, is straightforward..distributed pumping around the room-temperature beam tube. At liquid helium temperatures, the beam tube is a priori a superb "pumping" device because gas molecules strike the wall and attach themselves. But in the presence of the synchrotron light the molecules get knocked off again and drift slowly about, intercepting the beam.

To assess the seriousness of the effect, it was necessary to mount a series of experiments at the National Synchrotron Light Source (NSLS) at Brookhaven National Laboratory. Warm and cold tubes were evacuated and exposed to a beam of synchrotron radiation from the NSLS vacuum ultraviolet ring, adjusted in impinging angle to simulate the radiation in the SSC. (See Fig. 3-10.) Two mass spectrometers coupled to the dummy beam tube, as shown in the bottom half of Fig. 3-10 were used to analyze the gases desorbed by the radiation. Preliminary results indicate that some desorption of gases does occur, but that the effect will not cause difficulty. For example, based on these measurements, the luminosity lifetime of the beam from scattering by desorbed hydrogen gas (the most abundant of the various species) is estimated to be in excess of 150 hours. Since beam lifetimes from other causes are expected to be in the range of 48 hours, the contribution to beam decay from desorbed hydrogen is of little consequence. Other gases contribute negligibly.

Further experiments at Brookhaven and a parallel series in Japan with different beam pipe materials will provide additional data. It is clear already that photodesorption by the synchrotron radiation is not a vacuum problem requiring major attention.

\subsection{CONCLUSION}

The technical base for the SSC is provided on the one hand by the successful operation of large accelerators around the world, whether superconductivity is involved or not. The special nature of its magnets aside, the SSC is a cascade of accelerators of the type that is understood thoroughly in theory and in practice. It differs from its predecessors only in scale and in the numbers (but not the different kinds) of its technical components. In its use of superconducting magnets, it is not the pioneer. It builds on the experience of the Tevatron at Fermilab. The chosen magnet style, while different in some features from the Tevatron magnets, is a natural evolution in its most important part, the high-field, collared-coil assembly.

The ten-year R\&D program in th U.S. and aboard that led to the Tevatron and the intensified efforts of the recent past have established the technical basis for a realistic and accurate conceptual design and cost estimate. The remaining $R \& D$ is engineering development and optimization, rather than research to establish feasibility. 
61

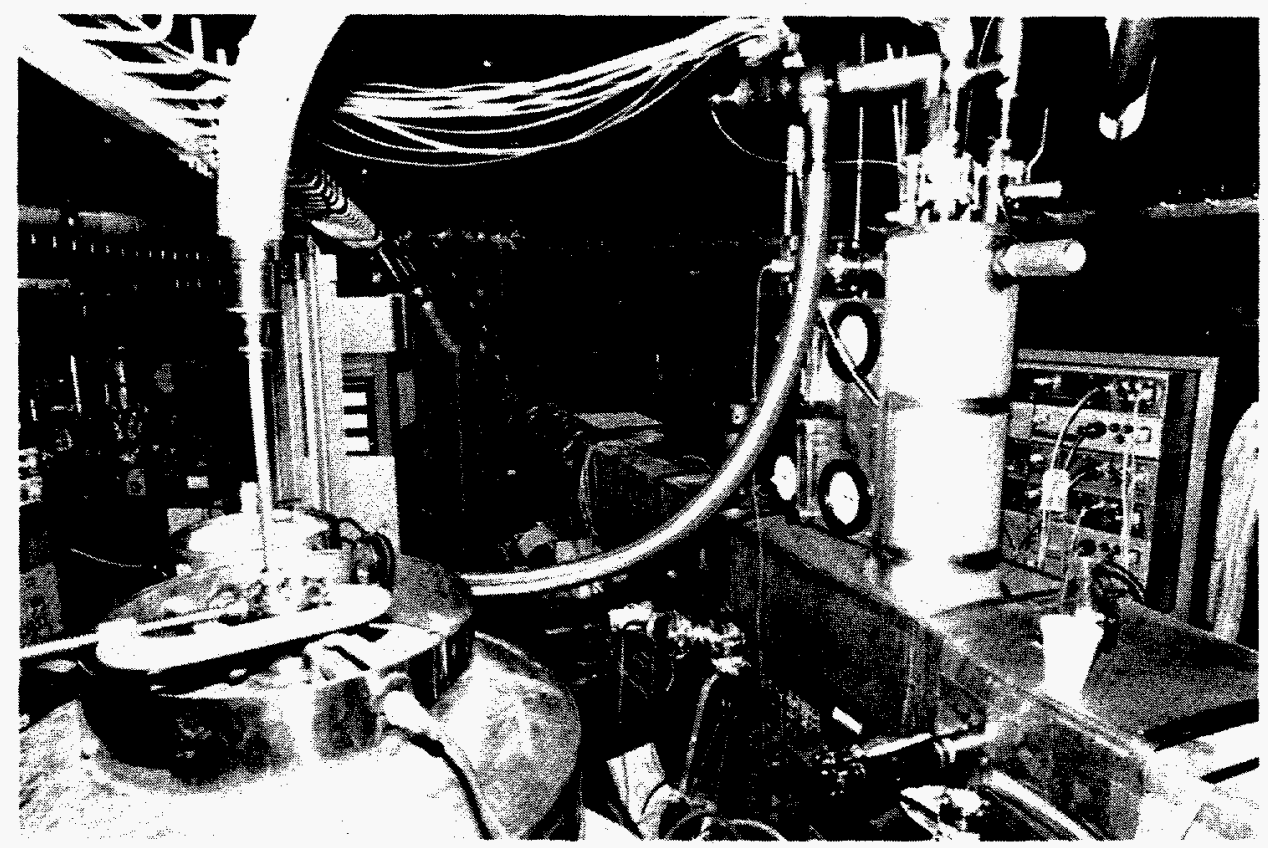

(a) Photodesorption experiment. Beam of synchrotron radiation comes from the rear into the rectangular box holding the cold beam tube and its cryostat.

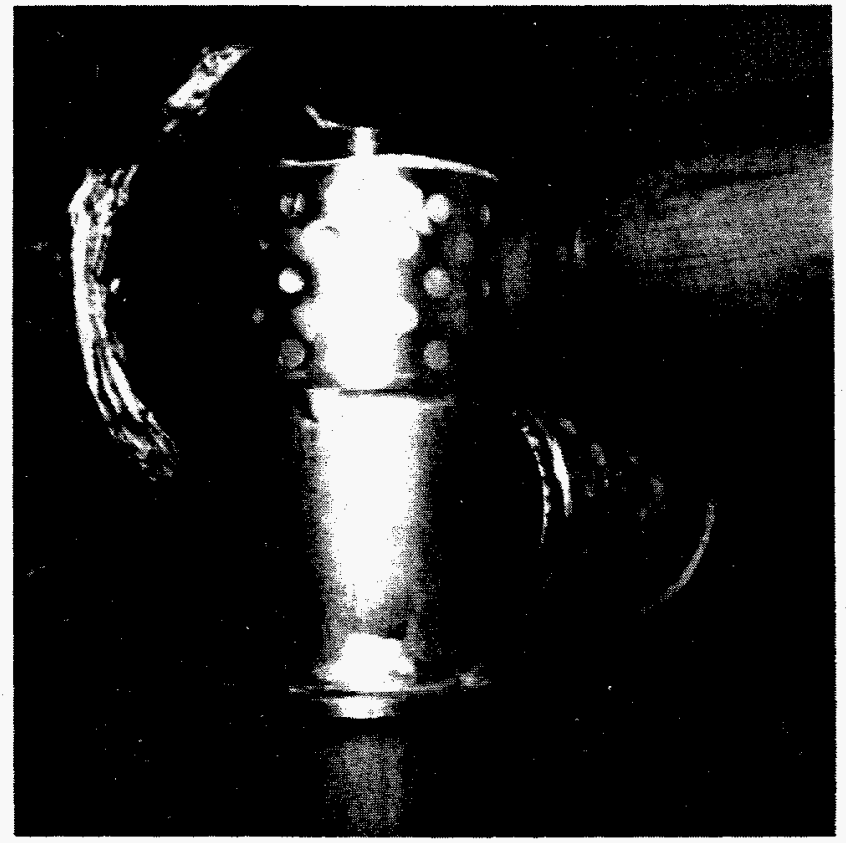

(b) Close up of the two mass spectrometers attached to the beam tube. These devices measure the amount and mass composition of the molecules knocked out of the wall of the beam tube by the synchrotron radiation.

Figure $3-10$. 
Chapter 4

SUPERCOLI.IDER FACILITY

\subsection{INTRODUCTION}

The central purpose of the SSC is to produce reactions among elementary constituents of matter at the highest possible energies. To this end, counter streaming beams of protons are caused to collide, almost head on. During collision a few of the protons will interpenetrate, allowing their constituents to interact. As the probability of proton interpenetration and reaction is comparatively low, the beams can be recirculated to collide repetitively for many hours without significant attenuation. Thus the SSC is constructed as a pair of storage rings capable of holding tightly confined proton beams on closed paths for a day or more without replenishment. The rings are made to cross at six locations where the collision reactions take place and where detectors which count and measure the reaction products for physics study are located. This arrangement is shown schematically in Fig. 4-1.

Note that the rings confining the proton beams are housed one above another in an underground tunnel that includes the interaction regions. The beams are guided around the desired path or orbit through an evacuated tube by a system of electromagnets. This magnetic confinement system consists of a periodic array of bending and focusing magnets, the bending magnets serving to establish the curvature of the orbit and the focusing magnets to confine the protons to a narrow domain about the desired orbit, as discussed in chapter 1 . Also depicted in Fig. 4-7 are an injector system and an accelerating system. As described in Sect. 1.6, the operating cycle of the SSC begins with the collider magnets maintained at low current for about thirty minutes while the proton beams are loaded into both collider rings. With injection complete, the acceleration system is activated. The slow increase in the beam energy is accompanied by a corresponding increase in the confining magnet strength thus keeping fixed the curvature of the beam orbit. This final synchrotron acceleration is complete in about fifteen minutes. The beams are then stored at collision energy, twenty times the injection energy, and steered into collision. The resulting reactions can be studied for a day or more before the cycle is repeated.

The injector systems consist of a source and linear accelerator, followed by three booster synchrotrons. The linear accelerator brings the protons up to $600 \mathrm{MeV}$ kinetic energy. The cascade of boosters then accelerate the protons successively to $7 \mathrm{GeV}, 100 \mathrm{GeV}$, and $1 \mathrm{TeV}$ kinetic energy. The final booster is a synchrotron with superconducting magnets; the others have conventional copper and iron magnets to permit rapid cycling. In addition to providing 1 TeV protons for the two main rings, the final booster synchrotron produces external beams for testing the response of detector components before their installation in the interaction regions of the SSC. 


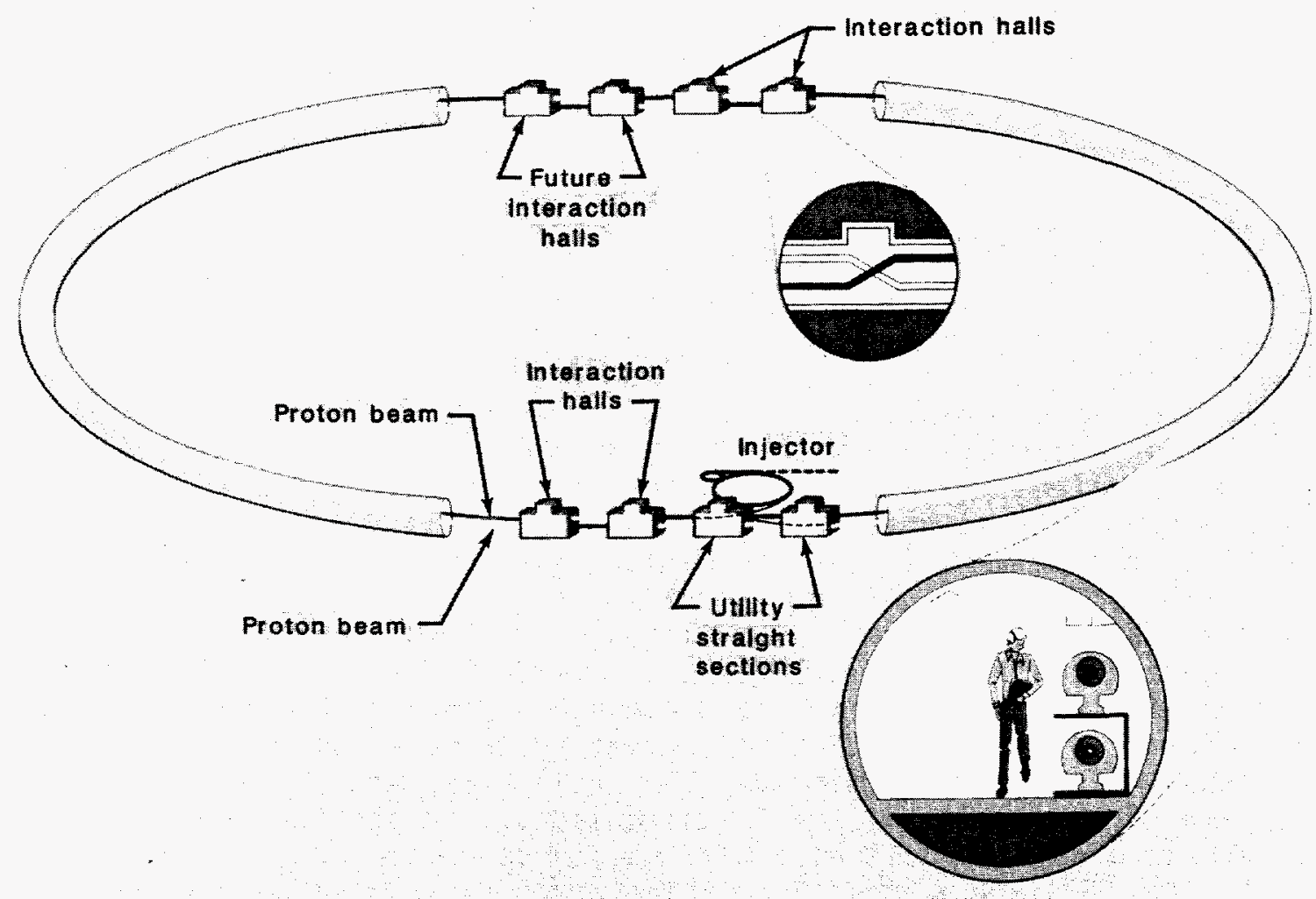

Figure 4-1. Schematic layout of the SSC. The large ring represents the underground tunnel that houses the two collider rings. The clusters of facilities provide for injection and acceleration of the proton beams, and for physics experiments in the six interaction halls (four to be developed initially). The tunnel cross section (lower right) shows the magnet rings, one above the other. The englargement inside the collider rings indicates how the oppositely moving beams of protons are made to collide. 
At collision energy the beams in the collider ring are quite small in cross-sectional dimension. In most of the ring the beam is a slim cylinder of about $0.1 \mathrm{~mm}$ in radius. At the collision points special magnets focus the beam to one-twentieth that size or five microns, about one-tenth the size of a human hair.

The ring is $83 \mathrm{kilometers} \mathrm{in} \mathrm{circumference.} \mathrm{In} \mathrm{the} \mathrm{curved} \mathrm{parts,} 86$ percent of the orbit length is occupied by the bending magnets. An isometric cutaway view of a supercollider bending magnet is shown in Fig. 4-2. A closer view of the magnetic components is given in Fig. 3-3 on p. 49. This inner body of the magnet is thermally isolated from its outer casing so that it can be maintained at its operating temperature of $4.35 \mathrm{~K}$ (269 Celsius degrees below zero) without undue refrigeration power being required. Design calculations and measurements of the SSC magnets show that the electric power required to operate the refrigerators of the SSC will be about 30 megawatts, comparable to or less than that being used to operate the largest existing accelerators.

The working fluid of the refrigeration system is liquid helium, as that is the only substance which maintains its fluid properties at the needed operating temperature. Cold liquid is introduced from refrigerators into the arrays of magnets at ten locations around the ring, cooling the superconducting coils as it flows through. At the end of an array it is withdrawn and sent back to the refrigerators for recooling. About 1.9 million liters of liquid helium are stored in the refrigeration system during operation. This impressive amount of liquid helium is still only about $1 / 30$ of recent U.S. annual usage.

In addition to the bending magnets, the continuous cryogenic envelope surrounding the beam vacuum chambers contains a focusing magnet about every 100 meters and special orbit and focusing correctors at the same interval, along with various pressure, thermal and electrical measurement and control devices. At less frequent intervals the cryogenic envelope contains valves and heat exchangers needed for the vacuum and refrigeration systems. Linking these all together with the injectors and refrigeration equipment and permitting the monitoring and control of the entire system is a network of computers connected by a broadband communication network and forming the collider control system.

The collider magnet system, its refrigerator and the attendant injector system, form the principal technical components of the SSC. Of them, the collider magnet system is dominant in bulk and in cost.

As previously noted, the rings and beam collision areas are housed underground. This provides a stable environment for the technical systems while at the same time containing the radiation associated with the operation of the beams. The required radiation shielding has been calculated independently by several investigators utilizing different analytic tools. The calculations have been verified by comparison with measurement at existing accelerators and with the very high energies available in the cosmic radiation. The results were reviewed by a panel of radiation safety experts drawn from the major U.S. and European accelerator laboratories.* Based on these careful assessments,

* Report of the Workshop on Environmental Radiation, (10/85) SSC Report SSC-SR-1016. 


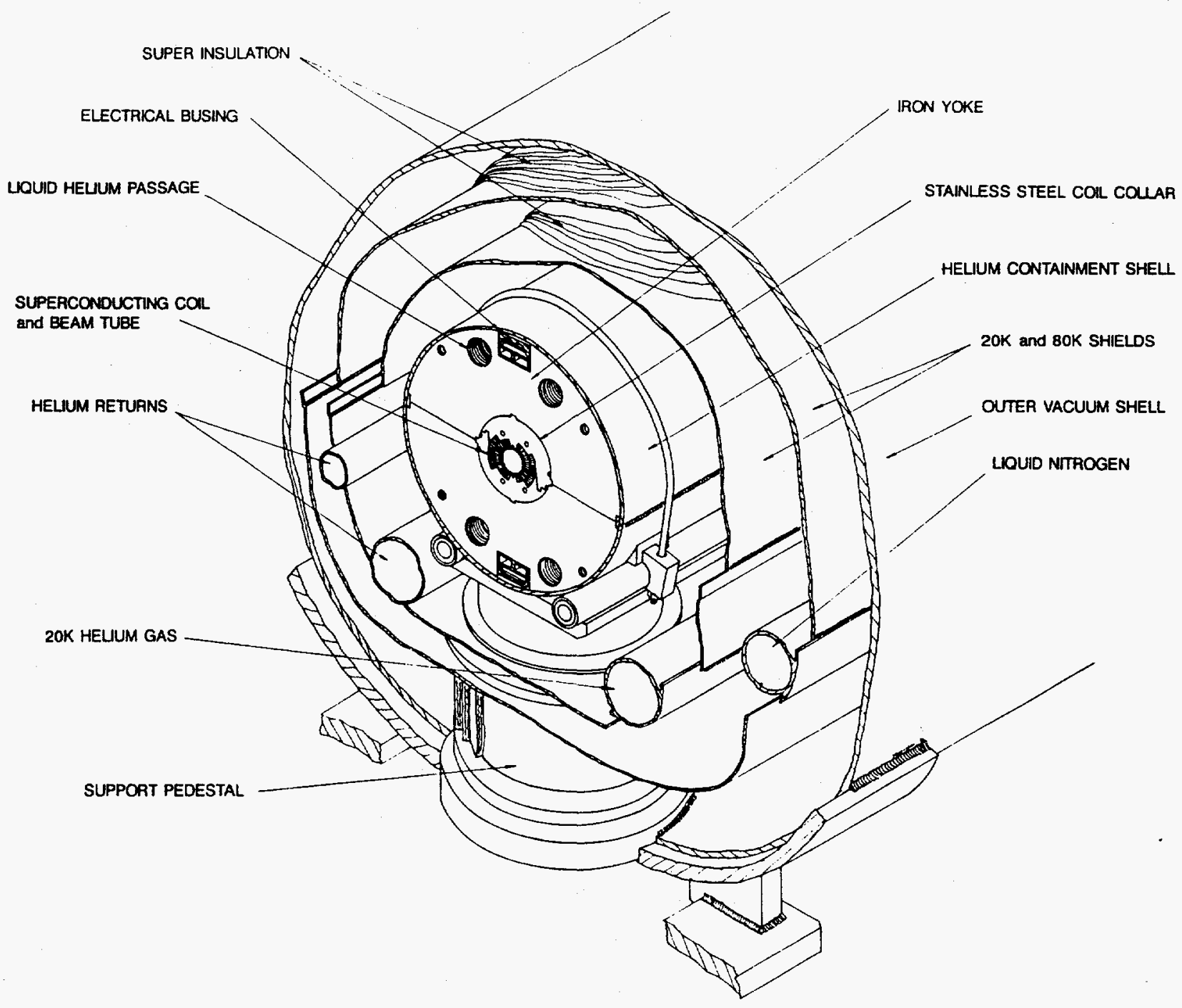

Figure 4-2. Isometric cutaway perspective drawing of the 6.6 Tesla SSC dipole magnet. 
twenty feet of soil above the top of the tunnel is adequate shielding even for a catastrophic loss of a full intensity beam. Paradoxically, although the SSC is a much higher energy device than any existing accelerator, because of its very long cycle time, the radiation problems associated with it are generally less than those routinely and safely dealt with at existing accelerators.

Surface buildings are needed to house the equipment used for the high energy physics experiments and the support functions such as shops and warehouses. In addition there must be housing for the injector complex and for the auxiliary equipment of the collider such as cooling and refrigeration equipment, power supplies and other utilities. While the experimental halls, injector, and general support buildings will be clustered together near the beam collision zones, ten above-ground utility buildings will be distributed uniformly around the periphery of the arcs. In all, it is anticipated that about 3000 persons will be at the facility at a given time. of these about 2500 will be staff and 500 will be visiting scientists participating in experimental and theoretical work at the facility.

A more detailed discussion of the physical layout of the collider rings and other conventional systems is given below in Sect. 4.4.

\subsection{PRINCIPAL PARAMETERS OF THE SSC}

In Chapter 2 the discovery potential at the TeV mass scale was discussed and the need for high luminosity was emphasized. The primary parameters of the SSC have been chosen to assure an extensive "discovery reach" into the region characterized by that mass and the expected cross sections. This parameter space is displayed in some of its aspects in Fig. 2-8 on p 41 . Studies of this kind, together with accelerator physics considerations, have led us to select a proton-proton collider with a design operating energy of $20 \mathrm{TeV}$ per beam and a maximum operating luminosity of $10^{33} \mathrm{~cm}^{-2} \mathrm{~s}^{-1}$ per collision point. To accommodate a wide variety of experimental techniques and detectors, provision is made in the design for six separate collision regions that can operate simultaneously under a variety of conditions.

Based upon economic studies and availability of advanced superconducting materials, an initial operating magnet field of 6.6 tesla has been chosen. The bunch spacing of $5 \mathrm{~m}$ and, to a lesser extent, a beam-beam tune shift of $10^{-3}$ per collision region (conservatively chosen, based on experience at the Spps collider) serve as a basis for deriving the other technical parameters of the accelerator. Some of the results are tabulated in the accompanying SSC Parameter Summary. Detailed arguments for the choices of specific parameters of the accelerator will be presented in the Conceptual Design Report. The following section presents a somewhat technical account of the actual layout of the machine and the confinement system.

\subsection{LAYOUT AND BEAM OPTICS OF THE SSC}

As the result of a number of studies involving accelerator physics, economics and operating efficiency considerations, it appears advantageous to 
SSC Parameter Summary

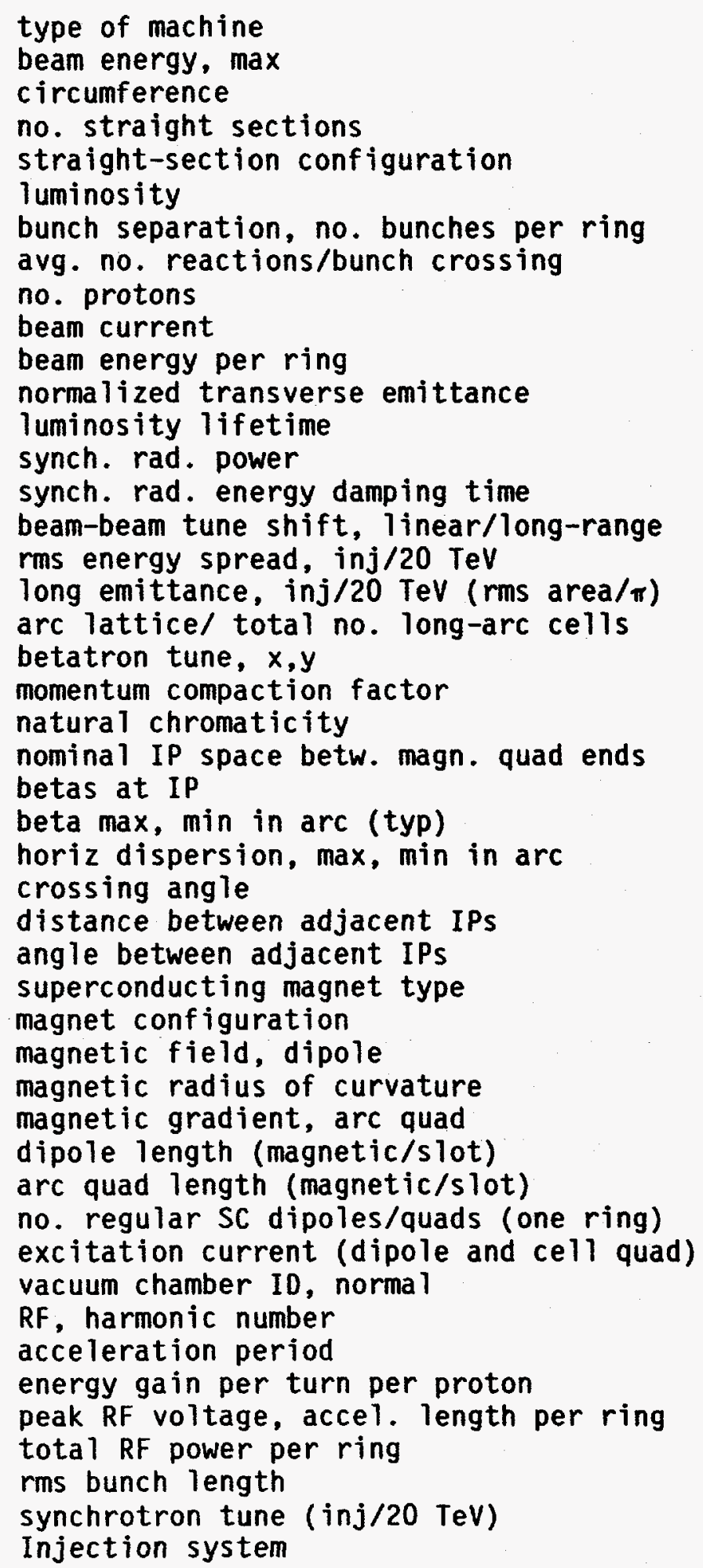

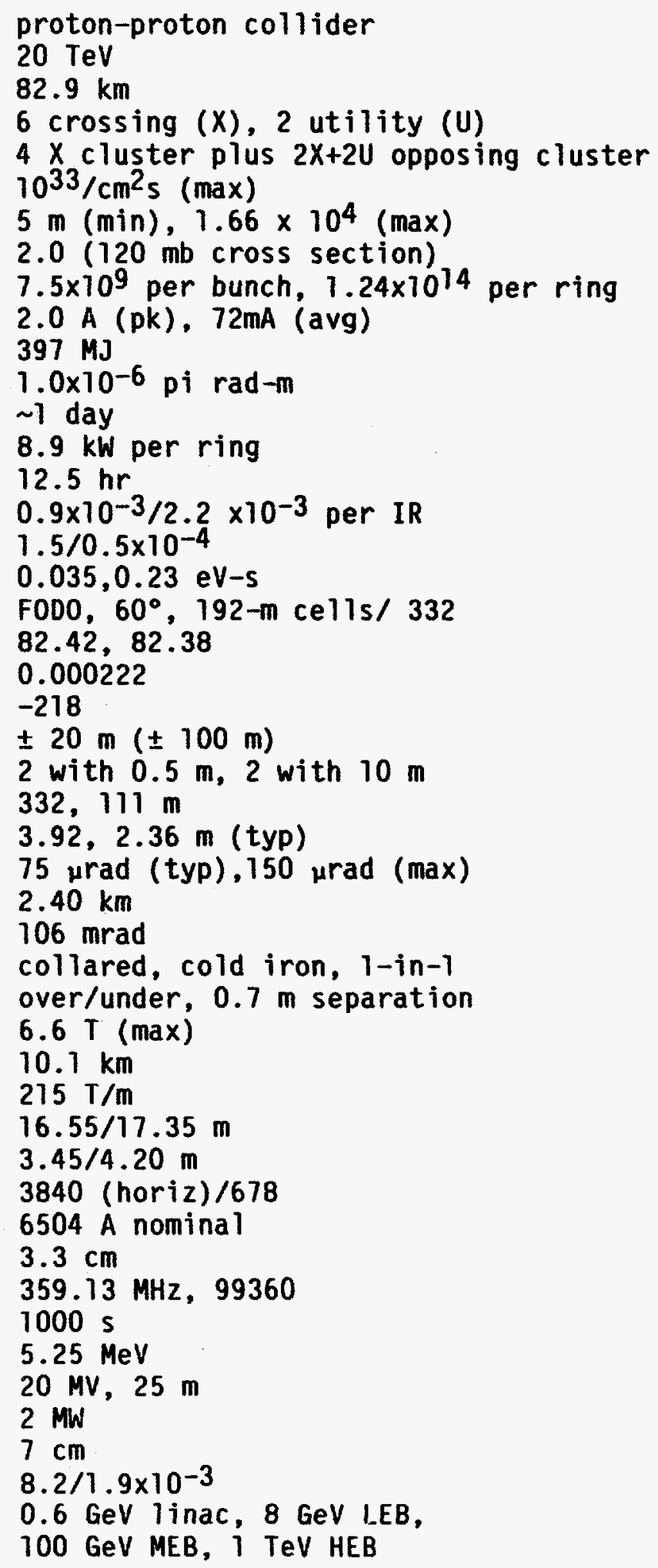


cluster the beam crossing regions into two groups incorporating both physics experimental areas and major supporting equipment. A plan view of the facility laid out in this fashion is presented schematically in Fig. 4-3. As indicated in the figure, refrigeration support buildings and tunnel access points are located at regular intervals in the ring arcs. Expanded plan diagrams of the cluster regions are shown in Fig. 4-4. Of note is the inclusion of bending in the spaces between collision points in order to prevent particles produced in one region from interfering with the study of collisions in another.

High and intermediate luminosity collision zones are being designed as part of the initial conceptual design study. It appears possible to structure the ring optics in such a way that a wide variety of crossing zones can be accommodated. This flexibility will permit modification of the crossing zones to optimize experimental usage over the life of the facility. Here we present some details of a high luminosity crossing zone which meets the need for crossing the beams at a very tight focus, separating the beams, adjusting the focusing of the confinement system between injection and storage conditions, and optimizing the matching of the crossing regions to the regular lattice. A vertical section at a crossing region, showing the optical components and the beam orbits, is presented in Fig. 4-5. In Fig. 4-6 we show the primary optical functions in the neighborhood of the crossing point, as adjusted for high luminosity operation. These betatron and dispersion functions are proportional to the vertical, horizontal, and energy oscillation amplitudes of the beam particles as they pass through the confinement system. Note that the optical components are separated in function, as discussed at a more rudimentary level in Sect. 1.6. (Fig. 1-3).

The lattice of Fig. 4-3 is made of three types of modules: arcs, interaction regions (IR) and the utility modules. Designating these three modules by $A, Z$ and $U U$, respectively, the SSC lattice consists of the following sequence of modules: $Z, Z, Z, Z, A, U U, W U, Z, Z, A$. (See Fig. 4-3, starting at $2: 30 \mathrm{p} . \mathrm{m}$. and going clockwise). The IR and the utility modules are interchangeable from the lattice design point of view. They both have length of $2400 \mathrm{~m}$ and both match at their ends to the arcs in the same way.

The IR's are arranged in two clusters, 4 IR's in one of the clusters, called the East cluster, and 2 IR's and 2 utilities in the other (West) cluster. The clustered IR's are adopted for the SSC because it is more cost effective to have experimental and utility regions sharing the same nearby support facilities.

On each side of an interaction region a triplet of quadrupoles rather than a doublet is used to provide the strong focusing needed at the interaction point. This has the advantage that the maximum $B$-function (proportional to the square of the amplitude of transverse oscillation) in the IR quadrupoles is minimized. Two interaction area designs employing the same optical scheme are included as examples in the conceptual design. The matching sections employed are flexible enough that many variants, with details arranged to accommodate particular physics experiments, are possible. The highest luminosity region has $\beta^{*} x=B^{*} y=0.5 \mathrm{~m}$ with $L^{*}=20 \mathrm{~m}$, the distance from the interaction point (IP) to the face of the first quadrupole. The maximum $\beta$ is $8 \mathrm{~km}$. In the other example IR, $L^{\star}=100 \mathrm{~m}$ and $\beta^{\star} x=\beta^{*} y=10 \mathrm{~m}$. 


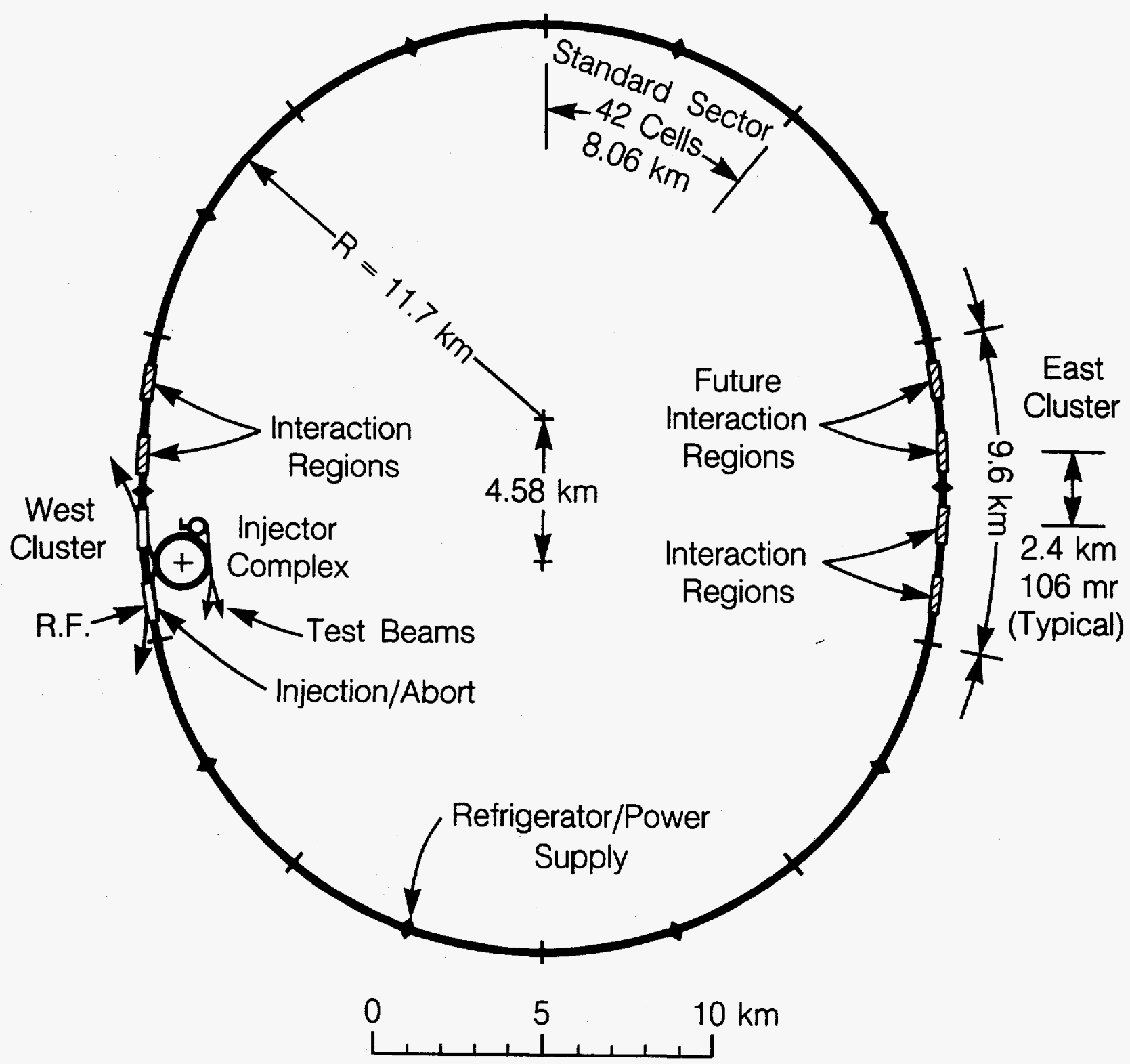

Figure 4-3. SSC Collider Ring Layout. East and West clusters are joined by arcs of $11.7 \mathrm{~km}$ radius. The East cluster consists of four interaction regions separated by $2.4 \mathrm{~km}$. The West cluster has two interaction regions and two utility straight sections (open rectangles) for injection and abort and for acceleration (RF). The cascade of synchrotrons that form the injector is inside the main ring at the utility straight sections. There are refrigeration and power units around the ring (black diamonds). 

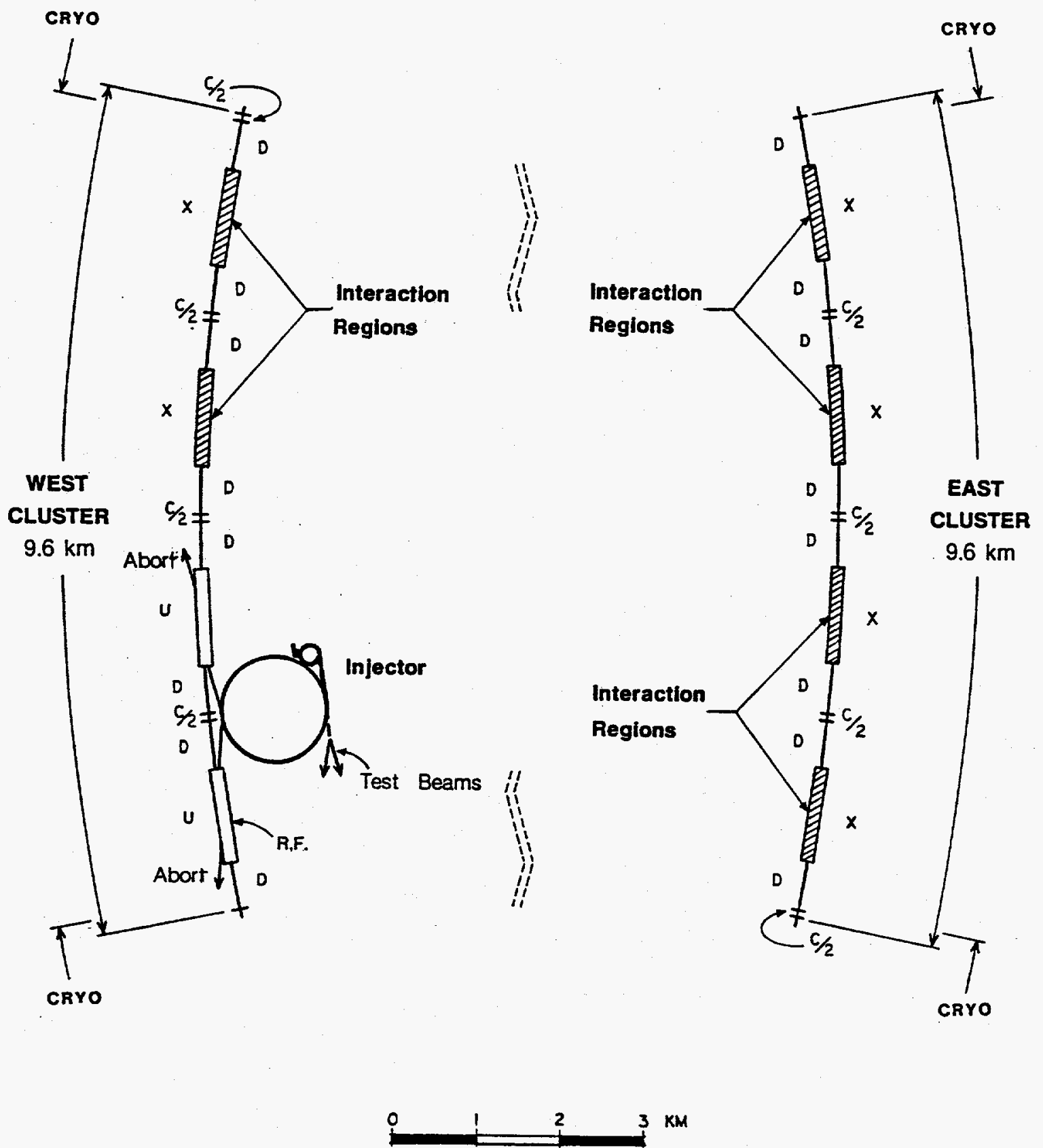

Figure 4-4. Enlargement of the layout of the East and West clusters. 


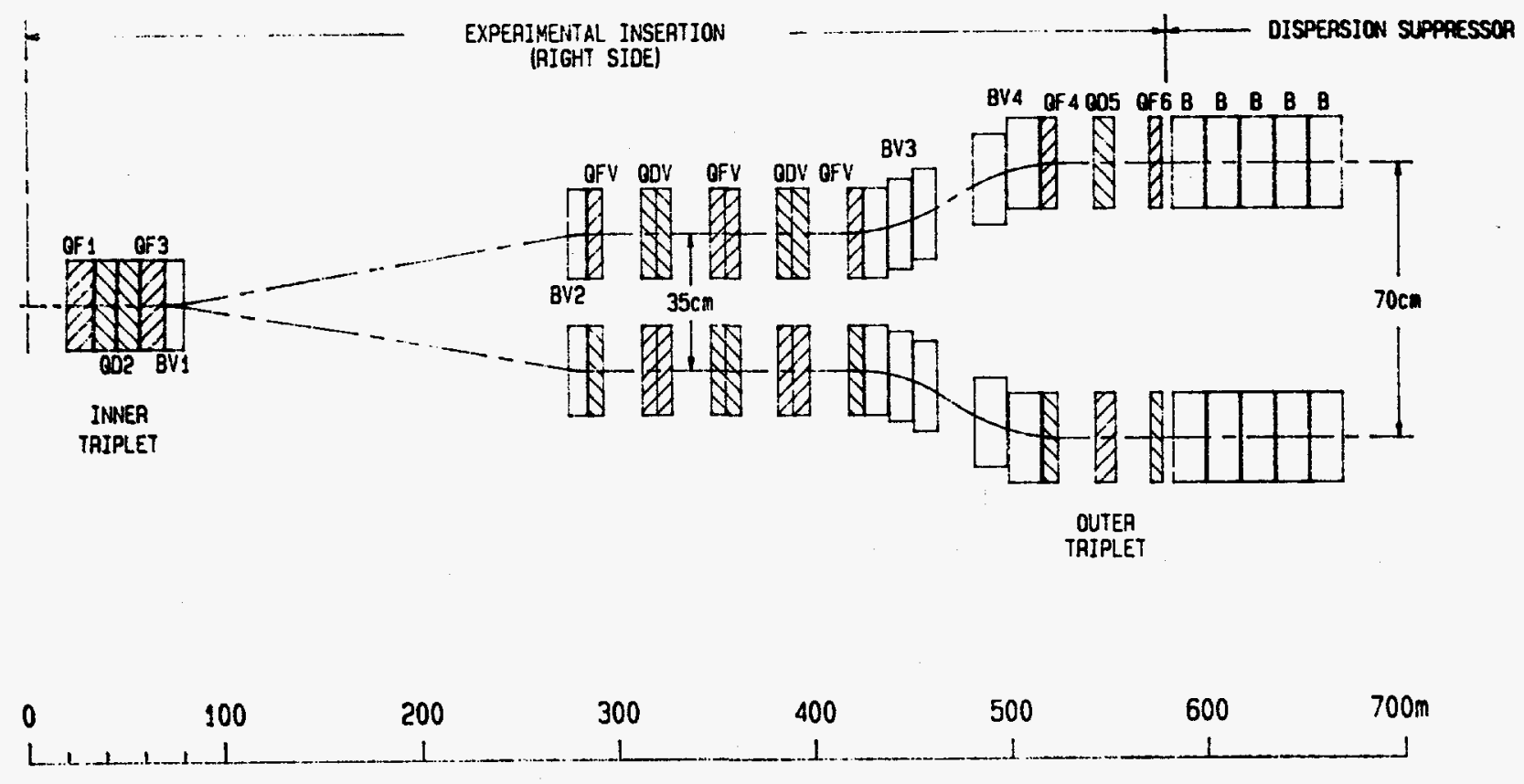

Figure 4-5. Geometry of the vertical beam crossing at a high-luminosity interaction point. Open rectangles represent bending magnets (horizontal or vertical); cross-hatched rectangles represent quadrupole focusing elements. The inner triplet of quadrupoles provides the final focus at the interaction point (IP). 


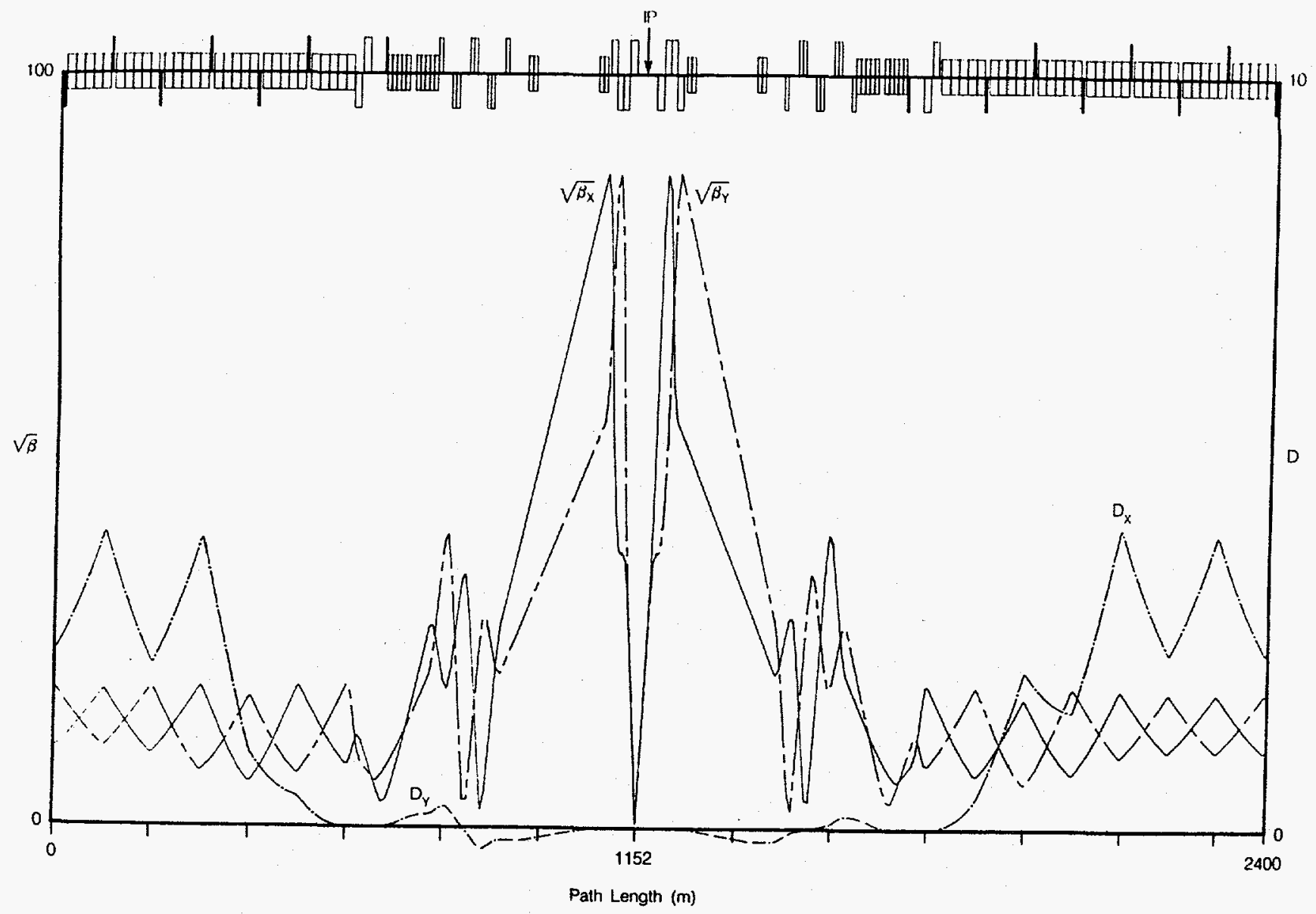

Figure 4-6. Optical functions $\sqrt{\beta_{x}}, \sqrt{\beta_{y}}, D_{x}, D_{y}$ of the SSC lattice near a high-luminosity interaction point. At top is a representation of the optical elements--bending (dipole) magnets are rectangles centered on the line, while focusing (quadrupole) magnets are rectangles above or below the line. The inner triplet of Fig. 4-5 can be seen near the interaction point (center of the line) with three quadrupoles (below, above, below), followed by the vertical bend dipole BVI (two centered rectangles). The solid rectangles on either side represent regular focusing quadrupoles with 5 arc dipoles between. 
The beams cross each other at a very small angle at the interaction points. The full angle is typically $75 \mu \mathrm{rad}$, but is variable from 0 to $150 \mu \mathrm{rad}$. As shown in Fig. 4-5, after the triplet (at the extreme left of the figure), the beams are separated vertically in two stages to a separation of $70 \mathrm{~cm}$. The optical elements are such that the properties of the beam are matched to the arc (or cluster) parts of the confinement system away from the interaction region.

The clusters are connected by arc modules, periodic arrays of bending and focusing magnets. As shown at the top of Fig. 4-7, each cell of the array consists of 10 bending magnets and two focusing quadrupole magnets each focusing (defocusing) in a separate plane. The cell length of $2 \times 96$ meters and cell focusing phase advance of $60^{\circ}$ were chosen to give minimum cost of the magnet system for the needed aperture. In addition to the bending and quadrupole magnets, each half cell contains a beam position monitor attached to the quadrupole and a correction magnet and cryogenic device unit, referred to as the "spool piece". There are 384 cells per ring in the regular arcs.

To secure proper functioning of the accelerator, a number of magnetic correction elements must be introduced: (a) Stability of the beams against the collective head-tail effect must be ensured by chromatic corrections effected with sextupole magnets placed in each spool piece. Most of these sextupoles are powered together in two families, although possible advantages of multiple family schemes are still being investigated. (b) Random manufacturing, powering, and installation survey errors of the dipoles and quadrupoles result in a requirement for independently powered dipole correction magnets to be housed in each spool piece. (c) Random departures from perfect uniformity of the dipole bending field also require a correction scheme to maximize the useful aperture and reduce resonance widths. To this end groups of normal and skew quadrupole, sextupole and octupole correctors are housed in certain of the spool pieces. (d) In others, skew quadrupoles are arranged to minimize error coupling of horizontal and vertical betatron motion. (e) 0ther groups of quadrupole corrections are provided for correction of cumulative focusing function and dispersion errors and adjustment of the tune. Tune adjustment capability of \pm 2 units is provided so that the optimum operating point can be explored experimentally. (f) Finally, distributed correctors for the sextupole and decapole components of the persistent currents in the dipole windings are provided. They consist of an array of wires, 200 micrometer in diameter, attached to the beam tube itself within each of the dipole magnets. These may be unnecessary if fine filament superconductor becomes available in time.

\subsection{CONVENTIONAL SYSTEMS}

The major feature of the SSC is the collider ring that is buried underground, and invisible. The shape of the ring is determined by the results of detailed physics calculations incorporating the capabilities of the required technical systems. For the current SSC design the two proton beams are contained within a tunnel having an overall circumference of $83 \mathrm{~km}$ (52 miles). Figure 4-8 shows the overall layout for the SSC.

The tunnel is composed of arc sections of uniform periodicity, interrupted by special sections called clusters. Within these clustered areas are found 


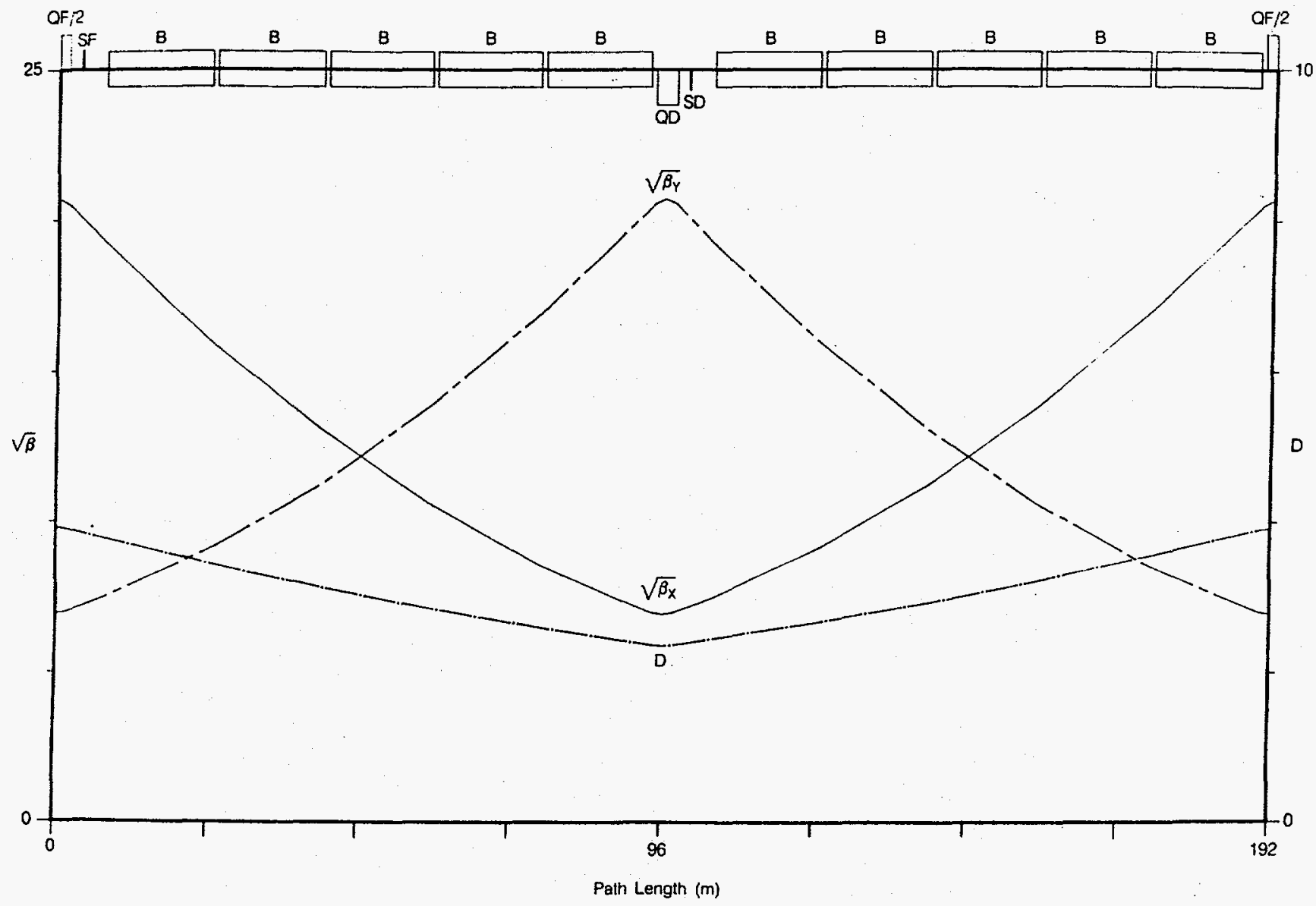

Figure 4-7. Optical functions $\sqrt{B_{x}}, \sqrt{B_{y}}$, and $D$ of a regular 192 meter cell of the arcs. At the top is the layout of the optical elements, in this case ten bending dipoles (B), two quadrupoles (QD and $Q F$ ), and two spool pieces (SF and $\mathrm{SO}$ ). 


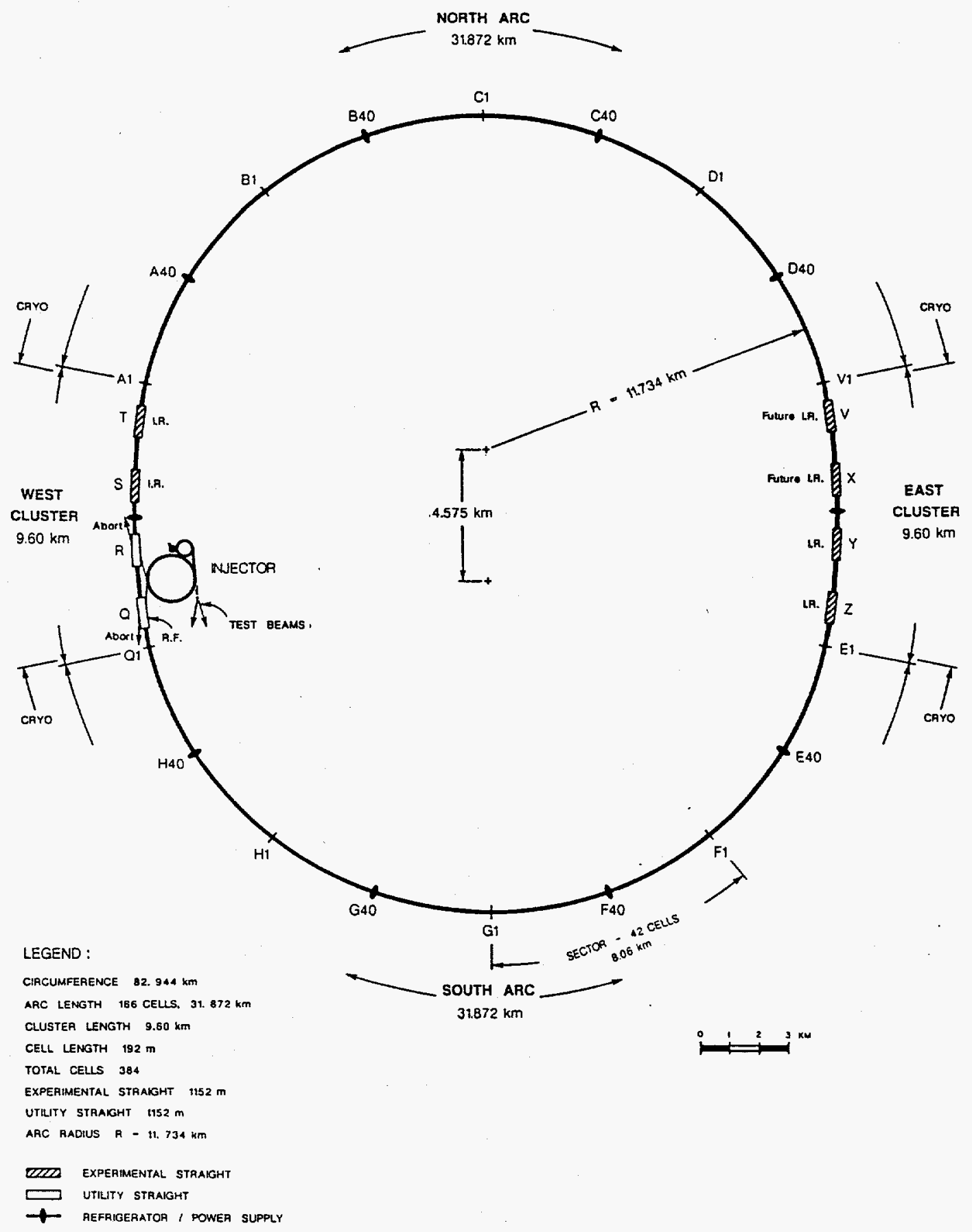

Figure 4-8. Site layout of the SSC. Major conventional facilities are labeled by letters or letters and numbers to be able to specify the type and location of components. The top to bottom distance is roughly $28 \mathrm{~km}$ (17 miles); the side to side, $23 \mathrm{~km}$ (14 miles). 
the experimental facilities as well as the utility sections needed for specialized accelerator functions such as injection, rf acceleration, and the beam abort/dump facilities.

The protons are introduced into the collider rings from the injector that resides adjacent to the collider. The last stage injector accelerator is capable of providing, separated in time, the clockwise and counterclockwise beams required for the collider. The transfer halls and the underground chamber around the Collider beams are part of the Injection Enclosure.

\section{(1) Siting Requirements}

The site must accommodate the accelerators and experimental facilities. The collider is conceived of as a planar ring 52 miles in circumference with the possibility of a slight tilt (up to 1 degree) to reflect the profile of the land. Up to 11,000 acres of land appropriately distributed around the ring are required. Other important attributes include:

* The site should be such that the SSC facility can be positioned, constructed, and operated in an environmentally acceptable way.

* The geology of the site must be such as to allow efficient tunneling or cut and cover construction with resulting structural stability and without encountering significant problems from the presence of groundwater.

* The site should provide year-round access by road, have a major airport nearby, and have adequate housing and educational facilities potentially available for the staff and visitors. Adequate industrial and construction resources are needed in the vicinity.

* The proposed site should have a source of adequate and reliable water (up to $2000 \mathrm{gal} / \mathrm{min}$ ) and electrical power (up to $250 \mathrm{MW}$ ).

* Accelerator operation requires avoidance of excessive very low frequency noise and vibration adjacent to the tunnels.

* Ease of construction and the cost and reliability of operation of the facility make desirable the avoidance of extremes of temperature and humidity.

(2) Generic Site Examples

Several geological scenarios have been studied as part of the Conceptual Design effort. Topographies and profiles representative of generic siles that exist at numerous locations in the United States have been assembled. One generic site consists of various layers of soil overlaying soft to hard rock. The plane of the tunnel has been sloped slightly to fit the surface topography. The depth of the collider ring has been set to provide a minimum of 20 feet of ground cover over the tunnel. Two additional geologic scenarios were carried through to completion for the purposes of estimating the costs for the underground facilities. These scenarios were for a deep tunnel in hard rock and a relatively shallow cut-and-cover tunnel in soft ground. 
(3) Collider Ring Facilities

Inside the tunnel are two rings of superconducting bending (dipole) magnets and focusing (quadrupole) magnets, which steer and confine two beams along approximately circular orbits in the arcs. Figure 4-9 illustrates the elements that might occupy the tunnel during construction and operation. For most of the circumference, the two beams travel in separate, parallel vacuum chambers, one above the other. Except for a warm gas return line, the cryogenic helium fluid used to cool the magnets is contained within the magnet vesse1. System valves are found every $960 \mathrm{~m}(3150 \mathrm{ft})$ where different sections of the magnet system can be isolated. At more frequent intervals (96 $\mathrm{m}$ or approximately $320 \mathrm{ft}$ ) there are pods of electronics for the instrumentation that controls the circulating beams. The usual services such as lights, ventilation, pumps, etc. are to be found in a typical tunnel section.

Connecting to the below-ground systems are a whole array of electrical cables and mechanical pipes. At the surface and distributed around the ring are ten refrigerator facilities (see Fig. 4-10) with large helium compressors. In the associated control rooms are found the power supplies that provide the current needed to energize the superconducting magnets, as well as one of the nodes of the accelerator control system. There will be a number of transformers and heat exchangers in the area to provide the services required by the technical systems. At two locations around the large ring are located major electrical substations connecting the accelerator complex to the power grid. Here power derived from overhead transmission lines is transformed to a lower voltage appropriate for the magnet power supplies and for distribution to sub-station locations in the acceleration complex. Other utilities such as water and sources of fuel will be provided as needed at the cluster areas and at the service areas around the ring.

\section{(4) Experimental Areas}

Allowance has been made for the construction of six collision halls surrounding the proton-proton beam interaction regions. Only four such areas are proposed to be constructed as part of the initial development of the SSC. AS shown in Fig. 4-11, a typical collision hall with a height of $15 \mathrm{~m}(50 \mathrm{ft})$ is envisioned to provide a central gallery $21 \mathrm{~m}(70 \mathrm{ft}$ ) by $21 \mathrm{~m}$, with smaller galleries at each end. At the side, behind a massive shield door, is an underground assembly area where detectors can be partially assembled before being introduced into the collision hall.

In one of the cluster areas will be found a research campus for the SSC. The campus complex may consist of fifteen or more buildings arranged in four major groups - laboratory, industrial, warehouses, and support buildings. The laboratory buildings will provide office and work space for the administrative and technical personnel. The buildings will contain the electronics development laboratories, control rooms, computing facilities, a cafeteria, meeting rooms, an auditorium, and other space for the purposes of the staff. Industrial buildings will house limited component assembly activities and associated offices. Warehouses serve as receiving and storage facilities. The support buildings - fire, site patrol, rescue and maintenance provides services to the entire SSC. 


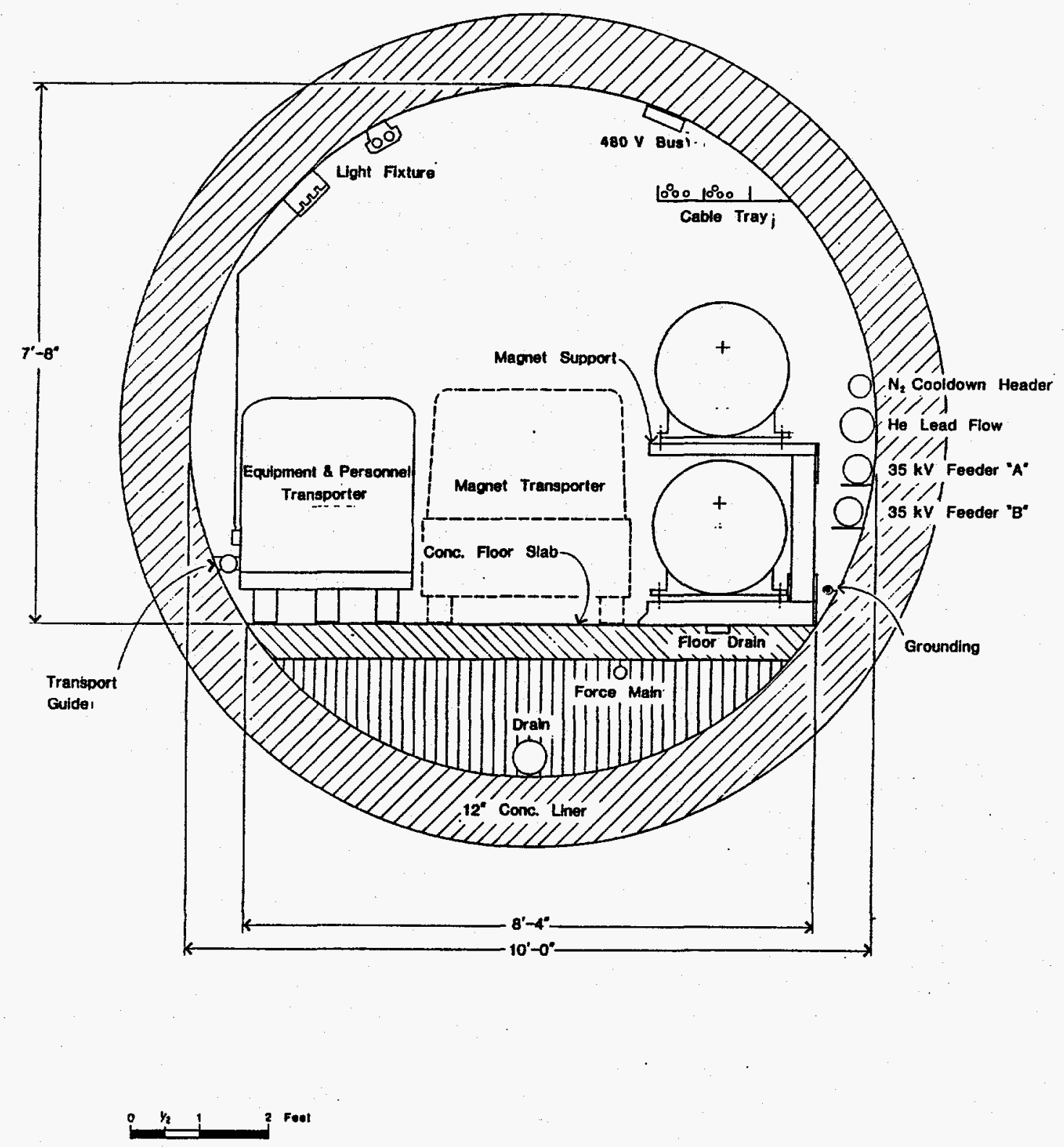

Figure 4-9. Cross section of tunnel in the arcs. The beams are separated vertically by 0.7 meters. The two rings of the confinement system are shown on the right. 


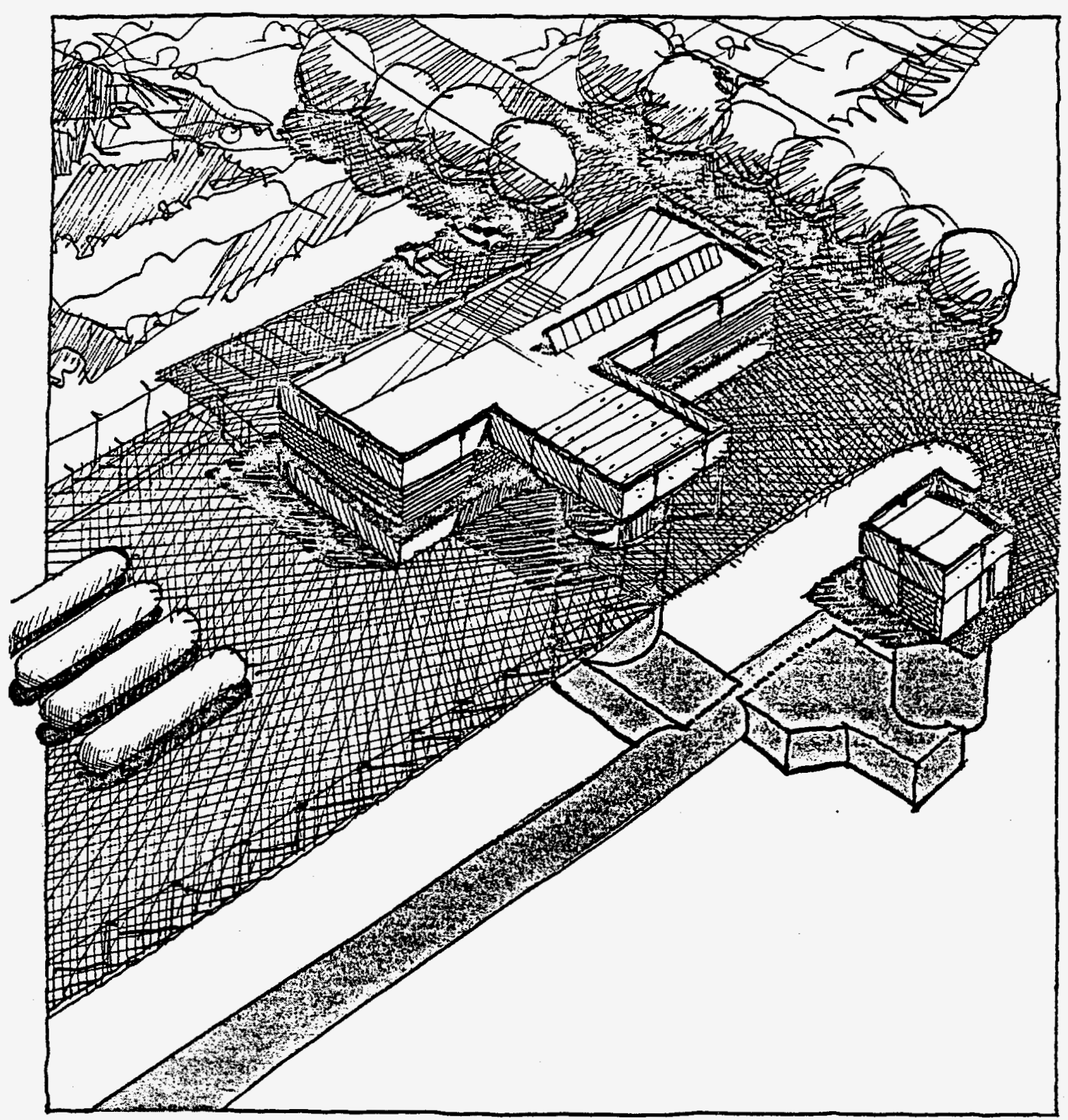

Figure 4-10. Aerial view of a refrigeration and power supply building with its access shaft to the tunnel below ground. 


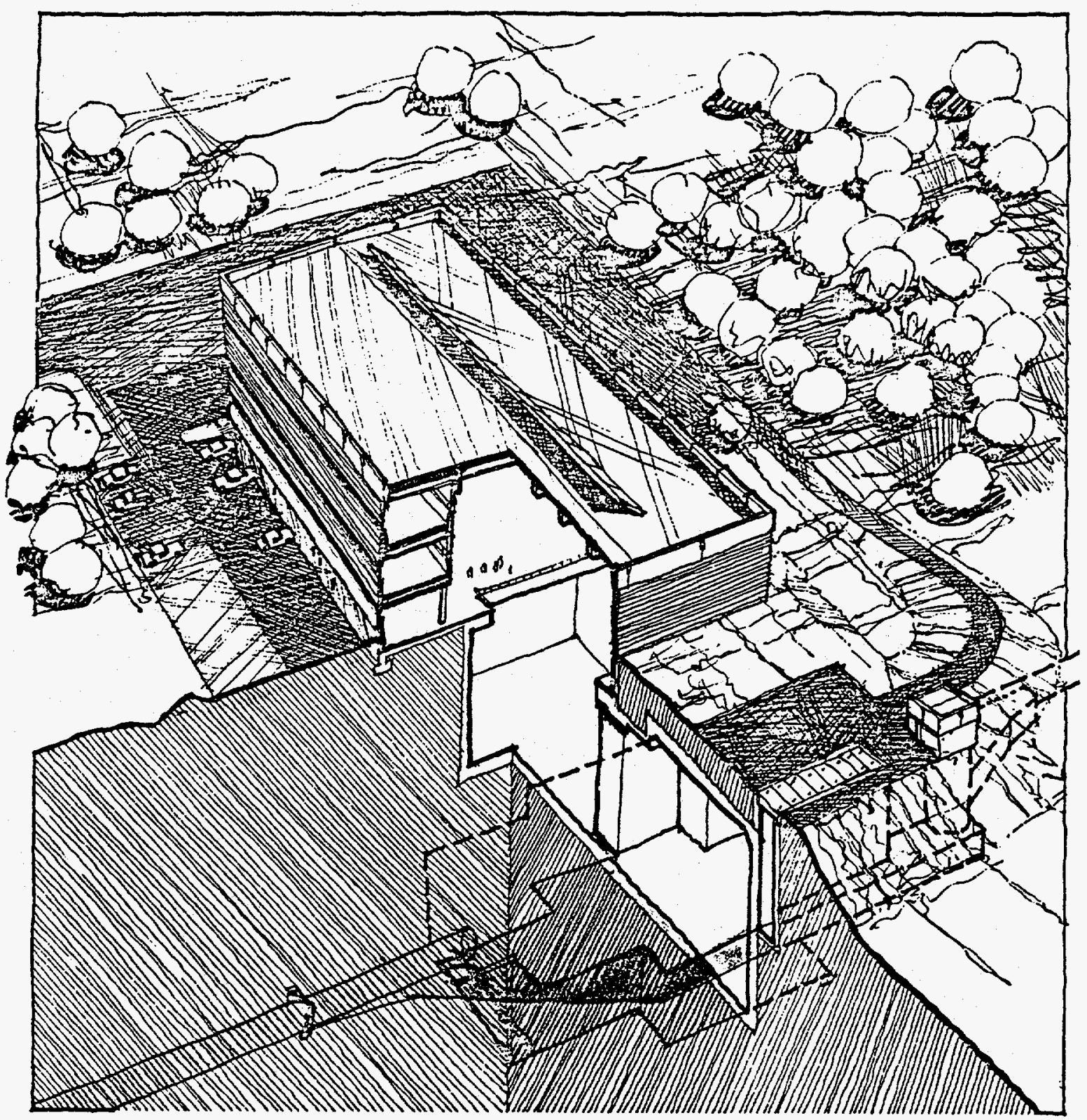

Figure 4-11. Aerial cutaway view of an interaction hall and staging area below ground, with the surface support building above. 


\section{(5) Safety and Radiation}

From the earliest discussions of the facility careful attention has been given to the various aspects of safety involved in its construction and operation.* The amount and types of radiation at the facility are not different from other accelerator facilities such as Fermilab, which operates as a facility accessible to the general public. In fact, in spite of the much higher energy involved in the SSC the cumulative amount of radiation will be less than that experienced at Fermilab, because of the very long cycle time of the SSC. As noted in Sect. 4.1, a great deal of care has been taken in determining the amount of shielding required to protect operating personnel as well as the general public from even the most unlikely accidental loss of control of the accelerated beam. In addition to the question of prompt radiation, detailed studies have been made to ensure that ground water in the vicinity of the accelerator will not be affected by its operation. The results of these studies are being incorporated into the design of the facilities.

During the pre-construction and construction phases of the project, an extensive measuring and monitoring network will be established to determine baseline conditions for the site. This network will be augmented as the facility comes into operation and will be the responsibility of a special environmental health and safety group within the laboratory. The Department of Energy requires that very detailed records be kept of all aspects of environmental concern, and that a detailed report of all these measurements be provided annually. Based on the experience of the existing accelerator laboratories, the SSC can and will operate well within the standards established by international bodies and incorporated into U.S. laW for radiation and industrial safety of both the staff and the general public.

of special concern at the SSC is the safety and security of remote and remotely operated large facilities some tens of miles from the main campus and control area. An extensive Supervisory Control and Data Acquisition (SCADA) system is being designed into the facility, utilizing the most recent developments in this area. This system will be a secure system reporting back to the main control room under 24-hour per day surveillance. Interlocks and trips will be incorporated to protect both personnel and equipment and to summon help as needed.

* Report of the $20 \mathrm{TeV}$ Hadron Collider Technical Workshop, M. Tigner, Coordinator, Corne 11 University, 28 March-2 April 1983 (Newman Laboratory of Nuclear Studies, Cornell University, Ithaca, N.Y. 14853). 
Chapter 5

TECHNICAL SYSTEMS

The concept of the SSC and its accelerator physics and conventional facilities aspects are described globally in the previous chapter. The designs of the technical systems follow from the parameters and operational goals that are stated there. Realization of those goals is constrained by the available technology, by likely scenarios of commissioning and operation, by the interrelations of the sub-systems, and by the desire to minimize the total cost. The present chapter describes the major technical components of the SSC and sketches the conceptual design of each. The detailed conceptual design and cost estimate will appear in the SSC Conceptual Design Report (April 1986).

\subsection{COLLIDER MAGNET SYSTEM}

The collider magnet system is comprised of the totality of the bending, focusing and correction magnets together with their associated cryogenic system. Of these by far the largest component is the dipole bending magnet complement.

\section{(1) Dipole Bending Magnets}

In all there are 7680 superconducting dipoles, mostly located in the regular arcs. The core of the magnet is shown diagrammatically in Fig. 5-1. The inner tube of $33 \mathrm{~mm}$ inner diameter forms the uitra high vacuum beam envelope. It will be a stainless steel or hard aluminum tube with high conductivity copper or aluminum coated on the inner surface to carry the beam image current with low impedance. On its outside may be wound the correction coil that may be needed to compensate for persistent currents in the ma in windings. Immediately outside this assembly is the two-layer main coil formed of superconducting cable with copper wedges interspersed to adjust the current density distribution for uniform dipole field inside the beam tube. The inner layer has 36 turns of 23 strand cable with $1.3 / 1$ copper to superconductor ratio and six wedges on each side, while the outer layer, which experiences a lower magnetic field than the inner layer, has 40 turns of 30 strand cable with 1.8/1 copper to superconductor ratio and two wedges on each side. Each dipole magnet uses 216 lbs of inner cable and 236 lbs of outer cable. The coils are held in place by a system of laminated stainless steel collars, $11 \mathrm{~cm}$ in diameter, that extend approximately $1.5 \mathrm{~cm}$ beyond the outer coil. The coil is compressed at a maximum stress of about 18,000 psi during collaring and the collars are keyed together, maintaining a prestress of about 6,000 psi. The collared coil assembly is surrounded by a yoke of low carbon steel, each dipole using about 6.5 tons of steel. This assembly is jacketed in a stainless steel skin which forms the vacuum-tight envelope containing the liquid helium coolant. Note the several passages provided for the liquid flow in the coil-yoke assembly. In addition to the cooling passages, channels for buses carrying current for the dipoles, quadrupoles, correction magnets and instrumentation leads are provided at top and bottom. 


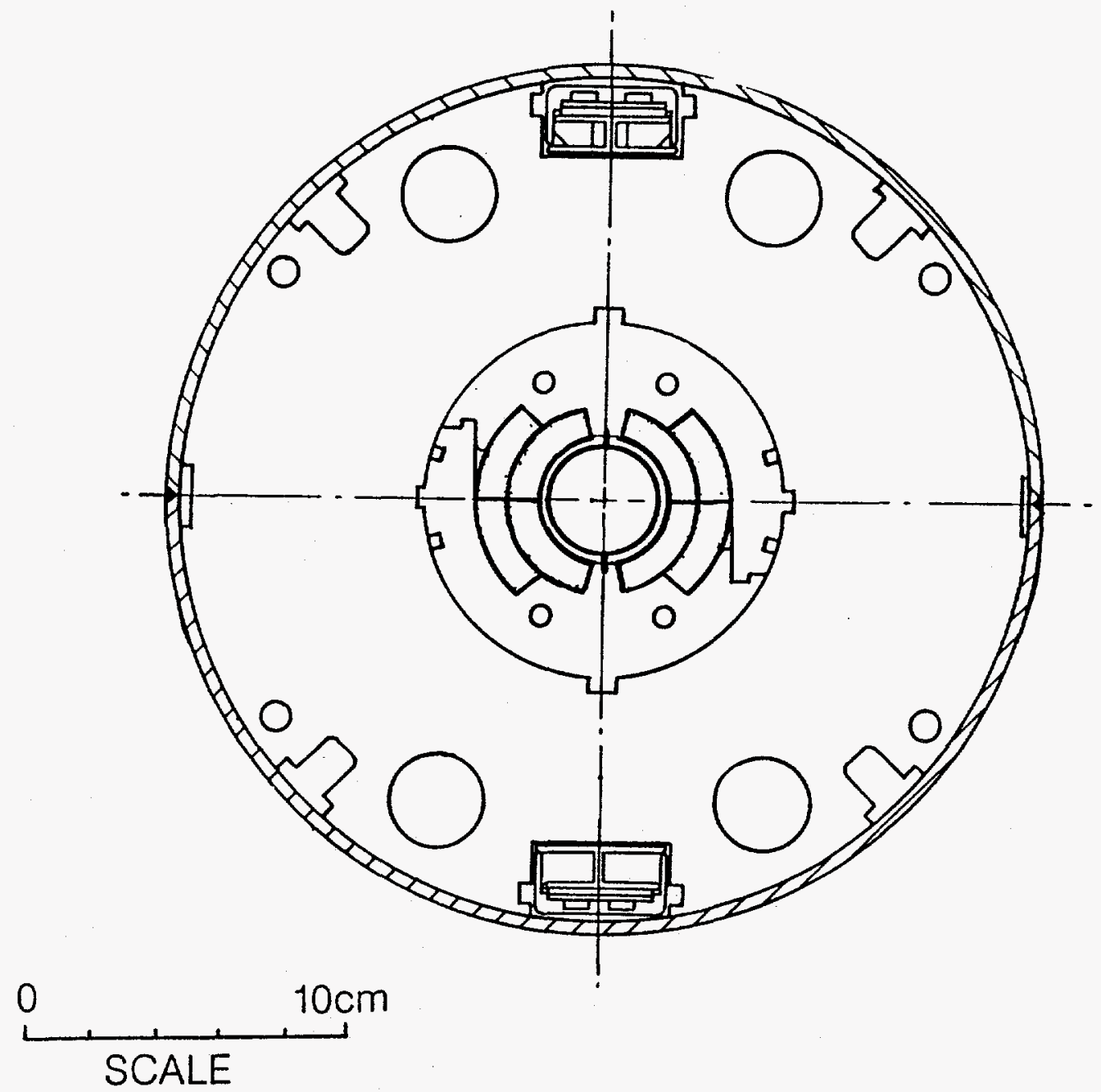

Figure 5-1. Cross section of the magnetic components of the SSC dipole. The double layer, cosine theta-style superconducting coil surrounds the central beam pipe. Around the coil package are the stainless-steel collars to hold the coils firmly in place. The outer iron yoke provides the return path for the magnetic flux. The four circles in the yoke represent passages for the flow of liquid helium. 
To form the complete magnet unit the yoked coil assembly is supported inside a cryostat, as shown schematically in Fig. 3-4 on $\mathrm{p}$. 50. In the $17.35 \mathrm{~m}$ continuous length of the dipole unit, there are five support pedestals which employ fiberglass-epoxy tubes for low thermal conductivity with heat intercepts at $80 \mathrm{~K}$ and $20 \mathrm{~K}$ to maintain low heat conduction into the liquid helium. To minimize radiative transfer of heat to the coldest magnet parts a system of graded shields is used. The innermost shield at $20 \mathrm{~K}$ faces the liquid helium container directly. Separating the $20 \mathrm{~K}$ shield from the $80 \mathrm{~K}$ shield is a blanket of multilayer insulation. A thicker blanket of multilayer insulation suppresses radiative transfer from the outer, room temperature wall to the liquid nitrogen temperature shield. On the basis of model measurements, heat inputs to the various temperature levels are estimated to be: $2.5 \mathrm{~W}$, including the synchrotron radiation (to $4 \mathrm{~K}$ ), $3.0 \mathrm{~W}$ (to $20 \mathrm{~K}$ ), $26 \mathrm{~W}$ (to $80 \mathrm{~K}$ ). Convective heat transfer is controlled by maintaining a vacuum in the space between the core and the outer shell.

Based on experience with models and expected performance of commercially available superconductor at the time of construction, a design operating field of 6.6 tesla has been set at a design operating temperature of the conductor of 4.35 K. The length of the dipole units in the design was determined by road transportation requirements to be approximately 17.3 meters.

Five dipole units are coupled together, mechanically, electrically and cryogenically between each pair of quadrupoles.

(2) Quadrupole Focusing Magnets

The quadrupole magnets which provide focusing for the beam are constructed as shown in cross section in Fig. 5-2. The quadrupole magnet has two layers of 30-strand cable identical to that used in the outer layer of the dipole with 16 turns in the inner layer and 22 turns in the outer layer of each quadrant. The inner layer has two wedge-shaped spacers in each quadrant to produce a current distribution that minimizes undesired field distortion.

The operating gradient of $212 \mathrm{~T} \mathrm{~m}^{-1}$ was selected using the same operating margin and material parameters as those of the dipole. The quadrupole magnets operate in series with the dipoles. The mechanical and cryogenic structure of the quadrupoles is very similar to that of the dipoles. The quadrupole length of approximately 3.1 meters is determined from the betatron phase advance needed to provide the requisite aperture.

\section{(3) Correction Elements}

Associated with each quadrupole is a correction and cryogenic unit spool piece. One design for the correction magnet is a multipurpose unit, the coil design of which is shown in cross section in Fig. 5-3. It is two meters long, and consists of a dipole, sextupole, and quadrupole from inside to outside. The coils are wound with $0.5 \mathrm{~mm}$ insulated superconducting strand of $2 / 7$ copper to superconductor ratio. The average current density in the coils is 400 $\mathrm{A} / \mathrm{mm}^{2}$, at a peak current of $100 \mathrm{~A}$., which is very conservative, since the peak field in the correction windings is less than three tesla. The complete package, with laminated steel, is $16 \mathrm{~cm}$ in diameter. The dipole is strong enough to correct at least five times the predicted rms closed orbit error. The quadrupole circuits are capable of a tune change of \pm 2 units; the sextupole circuits can change the chromaticity by plus or minus twice the natural chromaticity. 

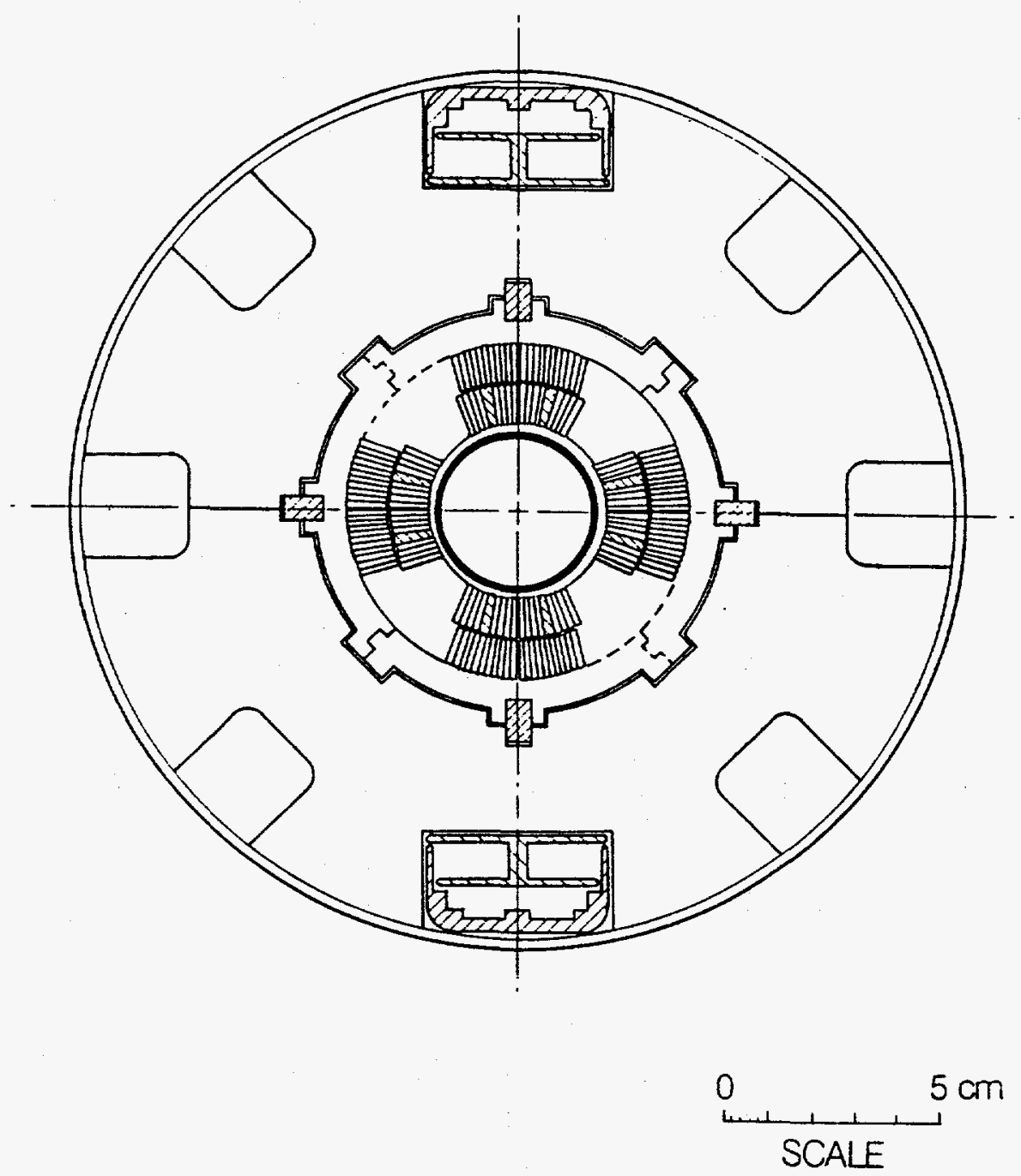

Figure 5-2. Cross section of the magnetic parts of the SSC quadrupole. The four-fold symmetry of the double-layer coil is apparent. Collars and iron yoke are similar to the dipole. 

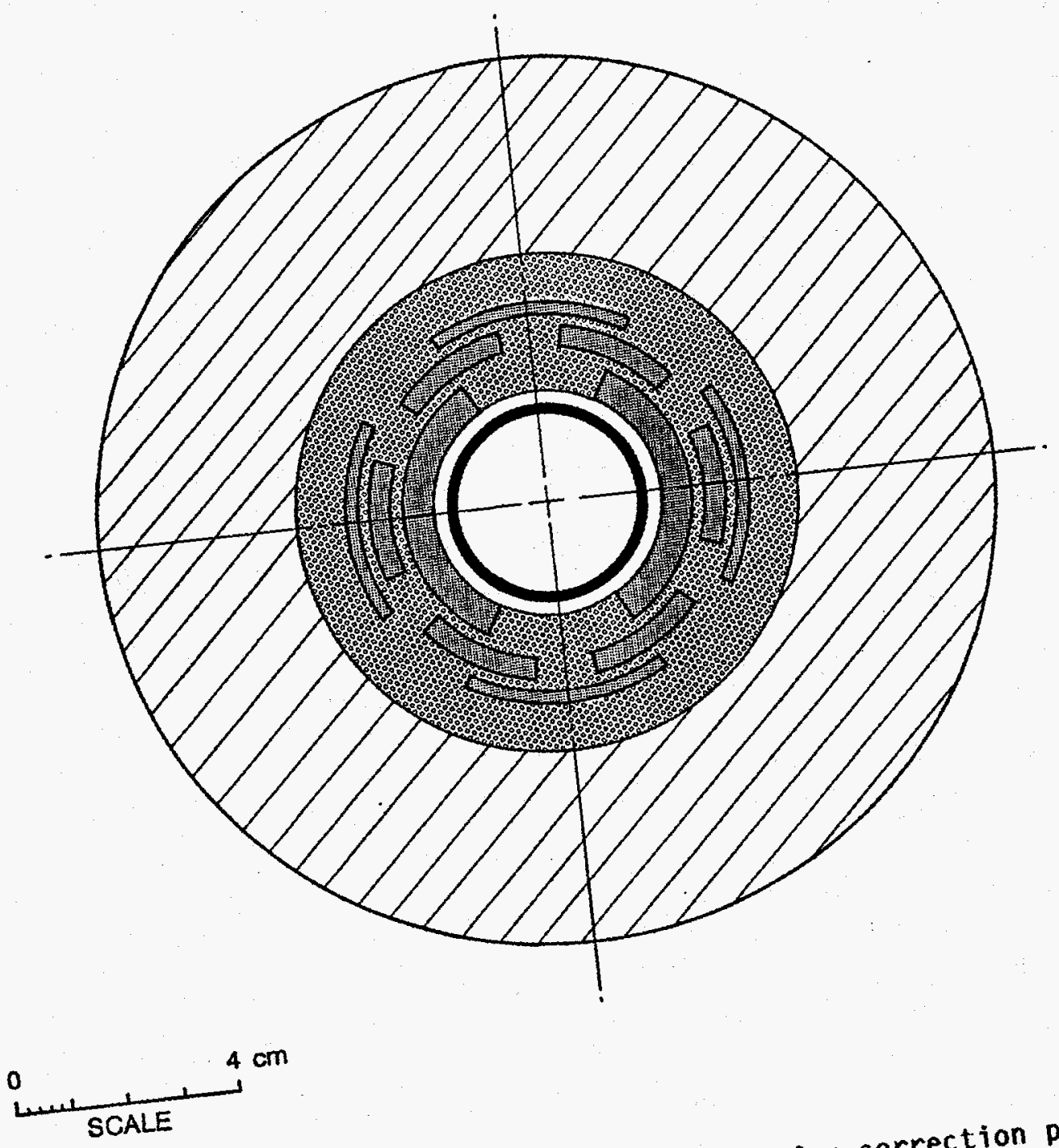

SCALE
Figure $5-3$. Cross section of coil configuration for correction package.
Dipole, sextupole, and quadrupole windings can be seen. 
Additional correction elements are placed at various locations in the rings for correction of the effect of random multipole errors. Each arc sector, for example, has sets of independently powered normal and skew quadrupole, sextupole, and octupole correction elements. These are strong enough to correct at least four times the expected rms errors.

\subsection{CRYOGENIC SYSTEM}

An extensive cryogenic system is required to maintain the magnets at the design temperature of $4.35 \mathrm{~K}$ or less. From a cryogenic point of view the machine is divided into 10 sectors, four in each of two arcs, and one in each cluster. The cryogenic loads are all roughly the same, requiring about two and one half megawatts of A.C. power for each helium refrigerator. In addition, there are two air separation plants to supply liquid nitrogen for the cryostat shields. They require $5.6 \mathrm{MW}$ each.

Each sector is divided into four cryogenic loops, a clockwise and counterclockwise loop for each ring, from the midpoint of the sector, where the refrigerator and main power supplies are located. The major components of a refrigerator are shown schematically in Fig. 5-4. They consist of a set of compressors for high pressure helium supply, a cold box that contains a heat exchanger and expansion engines, and the four magnet cryostat strings.

The principle of the cryogenic system is shown in Fig. 5-5. The refrigerator supplies about $400 \mathrm{gm} / \mathrm{s}$ of liquid helium at $4.15 \mathrm{~K}$ and four atmospheres pressure. The helium stream is divided equally into the four loops, warming as it removes heat from the magnets. At every cell the high pressure helium stream is directed through a heat exchanger (recooler), where it is cooled by a pool of boiling helium. The temperature of the boiling helium is set at approximately $4.2 \mathrm{~K}$ by the pressure in the cold gas return line. The temperature rise of the high pressure helium depends on the heat input and the flow rate, and is calculated to be less than $0.15 \mathrm{~K}$ between recoolers. At the end of each loop the helium stream becomes the supply stream for the low pressure (temperature) side of the recoolers. The returning boil off gas from the recoolers is the low temperature return fluid for the cold boxes of the refrigerator.

The magnet cryostats have two thermal shields, one cooled by helium gas at $20 \mathrm{~K}$, supplied directly from the helium refrigerators, the other cooled by liquid nitrogen at about $80 \mathrm{~K}$, supplied from the air separation plants and storage dewars at the helium refrigerators.

Each cryogenic loop is sub-divided into four sections, three $960 \mathrm{~m}$ long and one $1152 \mathrm{~m}$ long. A section is the smallest part of the machine that can be independently warmed to room temperature, and terminates at either end with a special spool piece that contains U-tubes for cryogenic connection from one section to the next, and a short piece of warm beam tube with a vacuum gate valve. Warm up is accomplished by isolating a section by removing U-tubes at each end, and turning on electric heaters inside the cryostat. If a magnet 


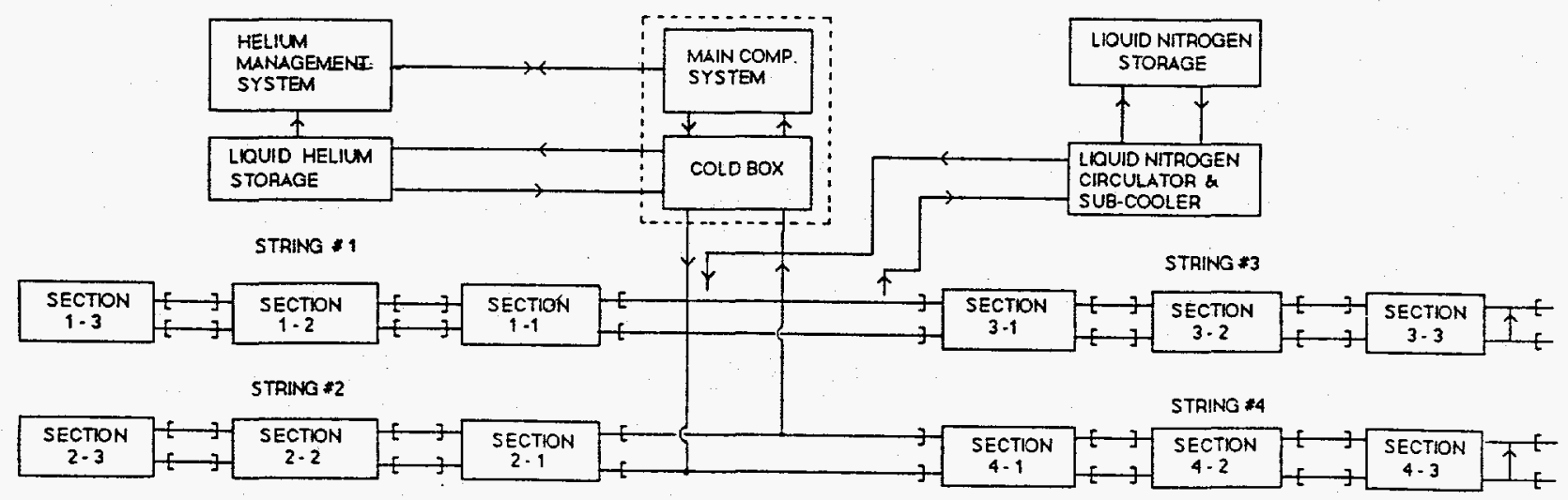

Figure 5-4. Block diagram of the major components of the SSC refrigerator system. The refrigerator cools two cryogenic magnet loops, which go 1/20 of the way around the collider ring in either direction from the refrigerator. 


\section{Ring number I cryostat}

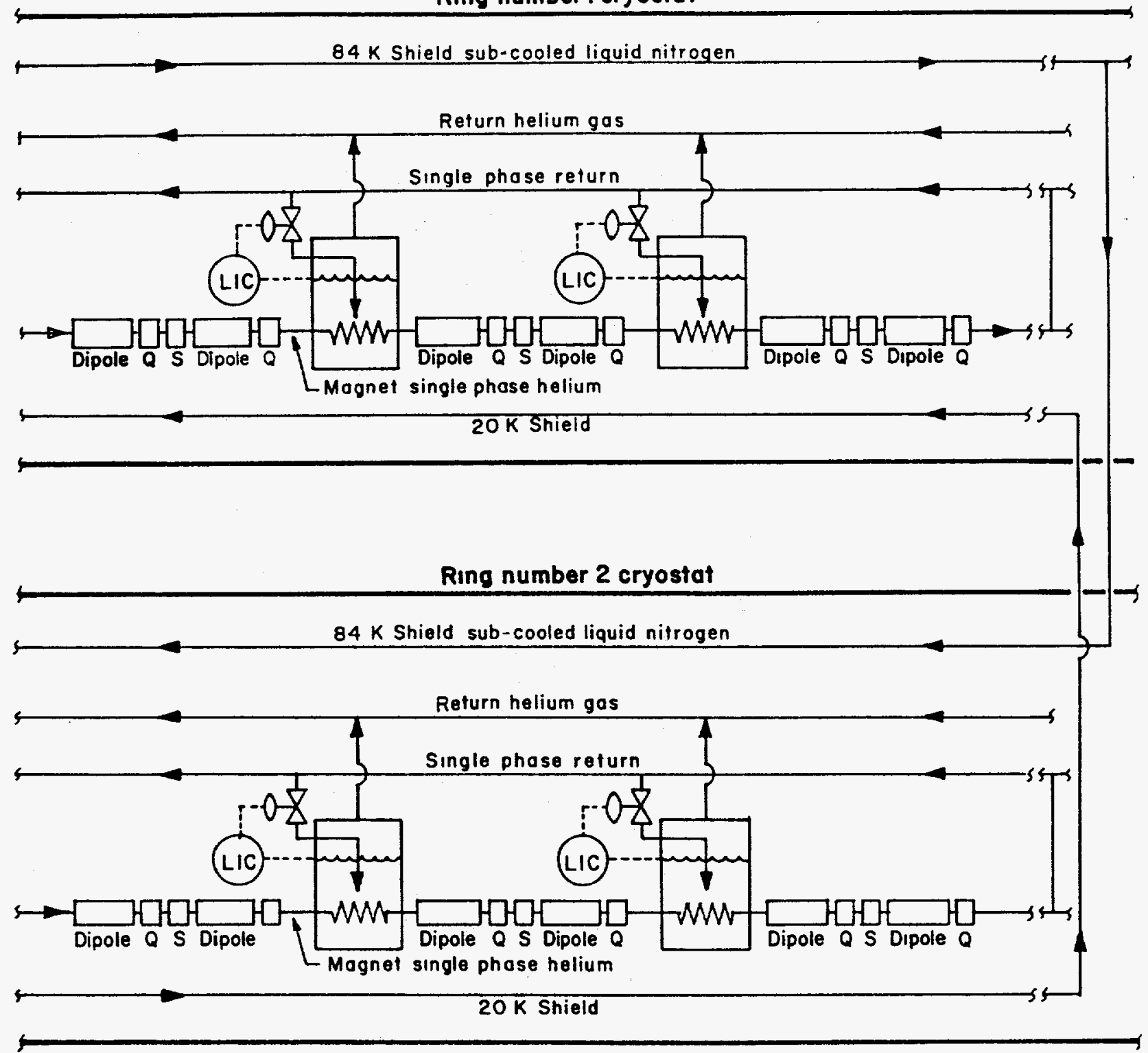

Figure 5-5. A conceptual representation of the SSC collider rings cryogenics system. 
has to be replaced, the entire operation of warming up a section, removing and replacing the device, and cooling down again is calculated to take about nine days.

The cryogenic systems of each sector are independent, but are connected one to the next through the magnets. In this way, neighboring refrigerators can be used to assist one another during cool-down, or to take over for a refrigerator that is malfunctioning. Each refrigerator is oversized by $50 \%$ for this purpose. All of the cryogenic transport is contained within the magnet cryostats. There is a single six inch pipe external to the magnets for the return of warm helium gas from the power leads, and from cool-down operations.

\subsection{OTHER COLLIDER SYSTEMS}

The magnets and the associated cryogenics systems are not the only technical components of the collider rings. There are power supplies to energize the magnets, quench protection circuits, a vacuum system for the beam pipe and the cryostats, a radio-frequency accelerating system, beam injection and abort systems, and ubiquitous controls. The requirements on these technical components and a brief description of their realizations are discussed in the following paragraphs.

\section{(1) Vacuum}

There are two vacuum systems of importance in the SSC. A very low residual gas pressure, about $10^{-12}$ atmospheres, is required within the beam tube to permit long beam life. A rather modest vacuum, about $10^{-7}$ atmospheres, is required within the space containing the core of the magnet to prevent significant heat transfer by convection. The primary pumping for both of these systems is provided by cryo-pumping. The gas molecules in the space will eventually hit a surface at $4 \mathrm{~K}$ and be frozen there. In both cases, the initial pump-down is accomplished with the use of portable carts that contain turbo-molecular pumps and diagnostic instruments. These use tunnel power, are connected to the cryostat or beam tube through hand valves, and are monitored through the control system. Once pump-out and cool-down are complete, the hand valves are closed, and the carts are taken away.

The insulating vacuum system has no permanently installed pumps or automatic valves. Vacuum gauges are permanently installed at each spool piece, and are read out through the controls system. The beam tube has ion pumps installed at every other spool piece. These are turned on before cool-down starts to obtain good vacuum, and are used during operation as gauges for monitoring the integrity of the beam tube vacuum.

The beam tube vacuum of the SSC is different from any other proton accelerator that has ever been built, because the synchrotron radiation desorbs gas molecules from the walls of the tube. Detailed studies of this process using synchrotron radiation from an electron ring impinging on a cryogenic beam tube has shown that the pressure rise will not significantly affect the lifetime of the SSC beams, as is described in Sect. 3.5 . 
(2) Main Power Supplies and Quench Protection

The dipoles and quadrupoles of a sector of each ring are connected in series to a single 6500 ampere, 300 volt power supply. Hence, each ring is independently powered, and has eight power supplies in the arcs. The rings are coupled together through the power supplies of the cluster regions, described below. Specifying the sector power supply at 300 volts permits the up and down ramp to full current to be done in 500 seconds, which will be particularly useful during testing and commissioning. The regulation from one sector to the others is done by differential transducers at the power supplies, and at the ends of each sector.

The power supply arrangement in the clusters is more complicated, because of the need to vary many of the quadrupoles used in the interaction regions. The mini-arcs between interaction regions are powered with the same program as the main arcs, as are the vertical bends. The inner triplet quadrupoles 1,2, and 3 on both sides of the interaction region, shown in Fig. 4-5 on p. 72, are powered from the main supply, but each one has a shunt power supply capable of $10 \%$ of the main power supply current connected to it. The same arrangement is made for outer triplet quadrupole 6 . The other members of the outer quadrupole triplet have their own 6500 ampere supplies, because the current varialion on those covers almost the complete range of current.

The outer triplet on one side of each interaction region is powered separately from the triplet on the other side because they are almost one kilometer apart, but the outer triplet in one ring is run together with the matching set in the other ring to save power supplies. If all the interaction regions are powered in this way, 16 power supplies are required for the cluster regions, and 20 more for the arcs, making a total of 36 high current power supplies in the SSC.

An active quench protection system illustrated in Fig. 5-6 is envisioned. A microprocessor at each half-cell measures the voltage across the half-cell, and sends the information to another microprocessor located in each section. The section microprocessor compares the voltages and determines whether there is a quenching half-cell. When a quench is detected, heaters are fired in the involved half-cell, power supplies are turned off, and dump resistors are switched into the power supply circuit. The decay time constant is 20 seconds for the ring as a whole. The bypass diodes in the current design are external to the cryostat in holes in the tunnel wall, and are connected to the magnets with pairs of leads at every spool piece. This arrangement may be required because the radiation level near the beams may be too high to allow diodes to be placed in the cryostats.

\section{(3) Injection and Abort Systems}

The abort system for one of the rings is immediately downstream of the injeclion for that ring in one of the utility regions. The injection and abort for the other ring are arranged in the same way in the other utility straight section. The High Energy Booster (HEB) accelerates beam in both directions, and is centered between the two utility straight sections, but out of the plane of the collider by seven meters. The proton beams are extracted tangentially from the same HEB straight section in opposite directions, and 


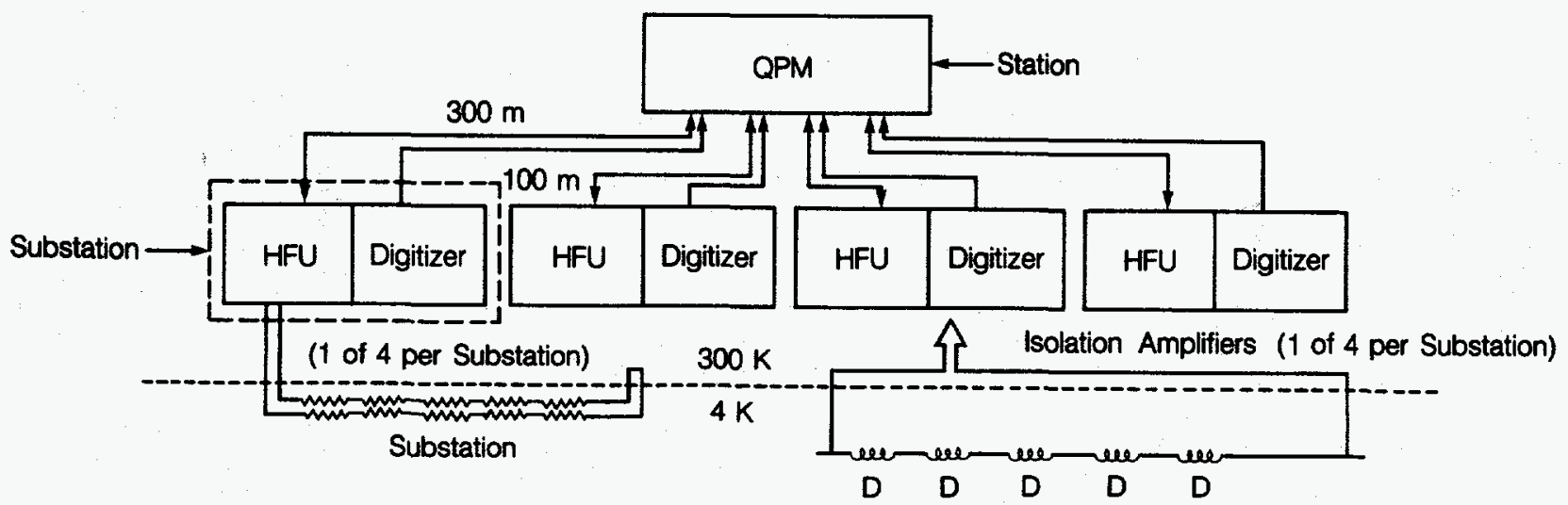

(a)

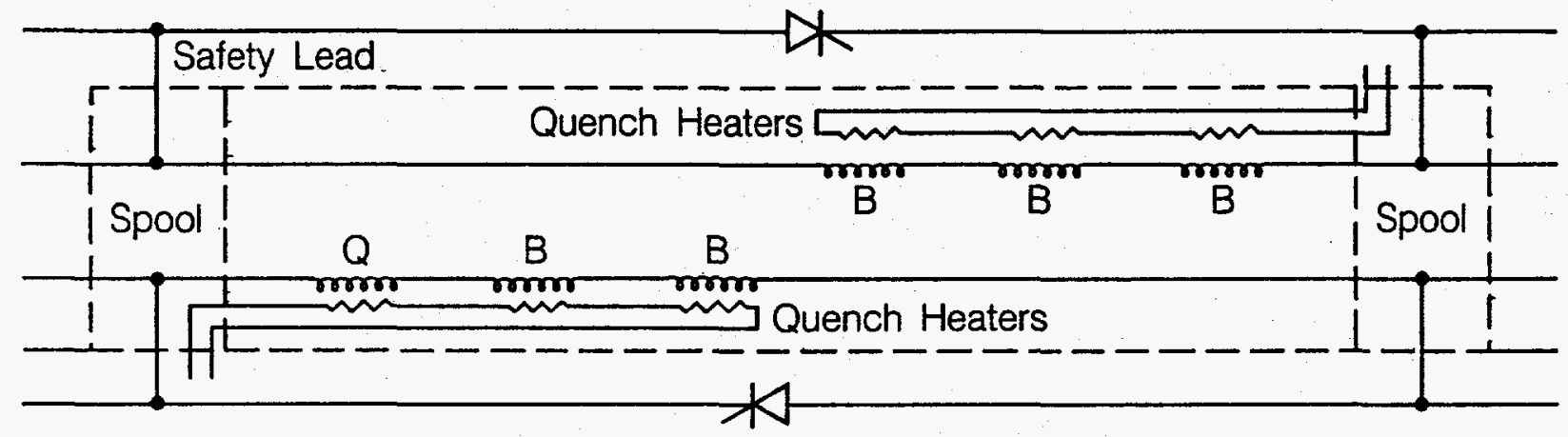

(b)

Figure 5-6. (a) Control logic for quench detection and monitoring, and the pulse circuits for the heaters. There are 12 HFU/Digitizer substations per quench protection monitor (QPM). (b) Schematic of the quench heater circuits and the by-pass protection diodes for a 96 meter half-cell. 
bent do:n lo the plane of the collider. Putting the two machines on different levels allows personnel access to one while the other is operating.

Since the abort is downstream of injection the beam can be steered directly into the collider ring abort dump, allowing complete tuning of the injection line before any beam is actually injected into the collider.

A full turn of beam is extracted from the HEB in a single turn and injected into the collider. This process is repeated 14 times to fill the collider rings completely, loading the batches box car fashion one behind the other, leaving a gap of four microseconds in the beam, which is sufficienl for the rise time of the abort kickers. With a HEB cycle time of 40 seconds, about 20 minutes is required to fill both rings, not including the time required to reverse the HEB and set up injection into the other ring.

The injection septa and kicker magnets are completely enclosed in a region with no focusing elements, permitting very simple tuning of injection. The kickers are divided into ten separate modules, so that if one (or even two) modules fail to fire properly the beam will circulate for enough turns to be properly aborted from the machine. If all the modules fail to fire, the beam will run into an internal beam dump in the central part of the utility straight section.

The abort system consists of a string of strong kicker magnets, each with its own pulser. The abort will tolerate the failure of one kicker module to fire. A double dog-leg bend using conventional magnels is set up across the abort straight section, so that beam losses are swept out before they can impinge on the superconducting magnets. The abort is generated by interrupting a pulse train that keeps the kickers from firing. The pulse train travels around the ring on a dedicated cable and can be interrupted by any device that has access to it, typically beam loss monitors, or quench protection. When the pulse train stops at the abort, a coincidence is set up with the gap in the beam, and the kickers fire only when the gap is present at their position, so that the abort kickers do not spray beam around the machine.

\section{(4) Controls System}

Because of the large size of the collider rings, the controls system must be distributed so that modules are close to the devices that are monitored and controlled. Most of this activity is near the quadrupoles and spool pieces, so the distributed modules are located in the tunnel at half-cell intervals. Because of radialion in the tunnel, these modules are placed in small shafts in the tunnel ceiling.

The controls resident in the Lunnel have as much local intelligence as possible, so that the communications load on the network is held to a minimum. All of the communication to and from any module at a particular half-cell goes through an intelligent crate controller at that location. In this sort of design, with local controls, power supplies, and intelligence, the only ringwide cables that are required are for the network.

A schematic representation of the total controls system is shown in Fig. 5-7. It consists of a large central multi-processor computer that 
contains the complete data base, and that feeds and receives information in one direction to control consoles, and in the other direction to a ring network. The next level of computer is at the 10 service buildings around the ring, and the control computers for other major systems, such as the injector complex accelerators. Those computers have a local data base, so that the network is not loaded down with data base transmissions. They perform the function of communicating to and from the distributed microprocessors in the tunne1, and are also capable of controlling the refrigerators and main power supplies, and run a console for local control, if that is necessary.

\section{(5) Radio Frequency System}

A small but very important part of the collider system is the acceleration system. Its function is to increase the kinetic energy of the beam from its value at injection ( $1 \mathrm{TeV}$ ) to its final storage and collision value (20 TeV) and to maintain the tight bunch structure of the beam during collisions while replenishing the $9 \mathrm{~kW}$ of power lost by each beam to synchrotron radiation. The technology for accomplishing this has been highly developed, particularly for use in electron accelerators. Indeed the SSC design studies show that systems developed recently for electron-positron colliders can be used directly for the SSC. Accordingly, the cavities and power amplifiers, together with their major controls, developed for the PEP collider have been adopted for the conceptual design.

A peak accelerating voltage of $20 \mathrm{MV}$ per ring at an operating frequency of $360 \mathrm{MHz}$ meets the requirements. This voltage can be readily produced with eight of the PEP cavity units driven by two of the PEP klystrons. The acce1erating cavities themselves, eight units, occupy a space along the beam line of only 25 meters. Assuming the tunnel is not too deep, the four klystrons (total) needed to drive both rings would be located above ground over the part of the utility straight section where the cavities are installed. In each ring there are two accelerating units, each containing one klystron. A schematic representation of an accelerating unit circuit is shown in Fig. 5-8. A photograph of a PEP cavity is presented in Fig. 1-5 on p. 20.

\subsection{INJECTOR SYSTEMS}

In order to maintain good availability of the main collider, an injector which can load the ring in a small fraction of its design operating cycle period is required. One half hour has been selected as the total filling time for both rings. As high beam brightness (low emittance) is required for economical operation of the SSC main rings, the injector chain must also be designed to preserve the source brightness during acceleration to the SSC injection energy. Accordingly, an injector chain which avoids all transition energy crossings and limits the space charge-induced tune shift to 0.25 or less has been designed. These requirements can be met with a four component injector chain consisting of a linac followed by a cascade of three synchrotrons shown schematically in Fig. 5-9. Note that the geometry is so arranged that only the high energy booster, which will utilize superconducting magnets, will carry bi-directional beams. The primary parameters of the injector chain are displayed in the Table 5-1. 


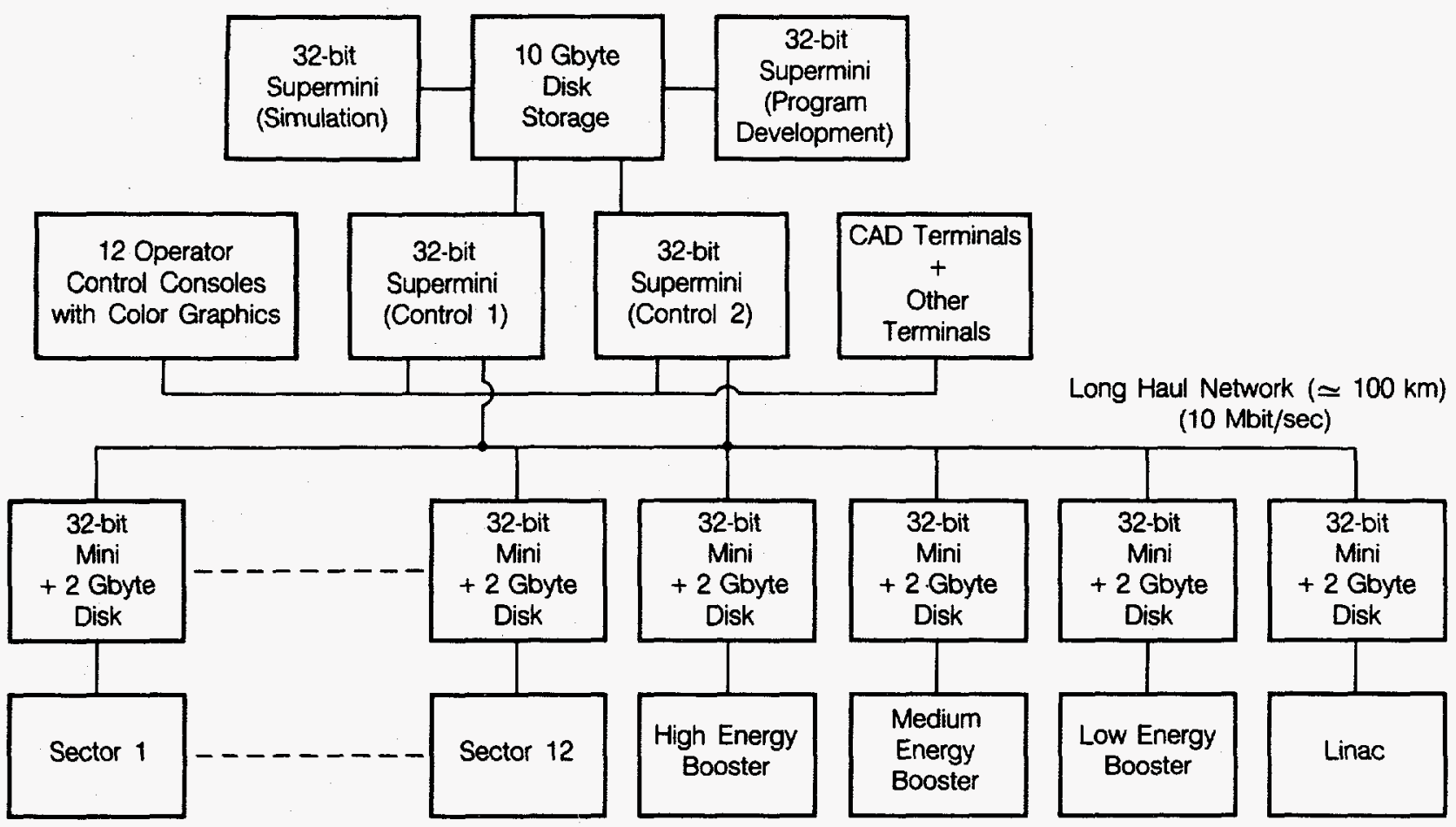

(a)

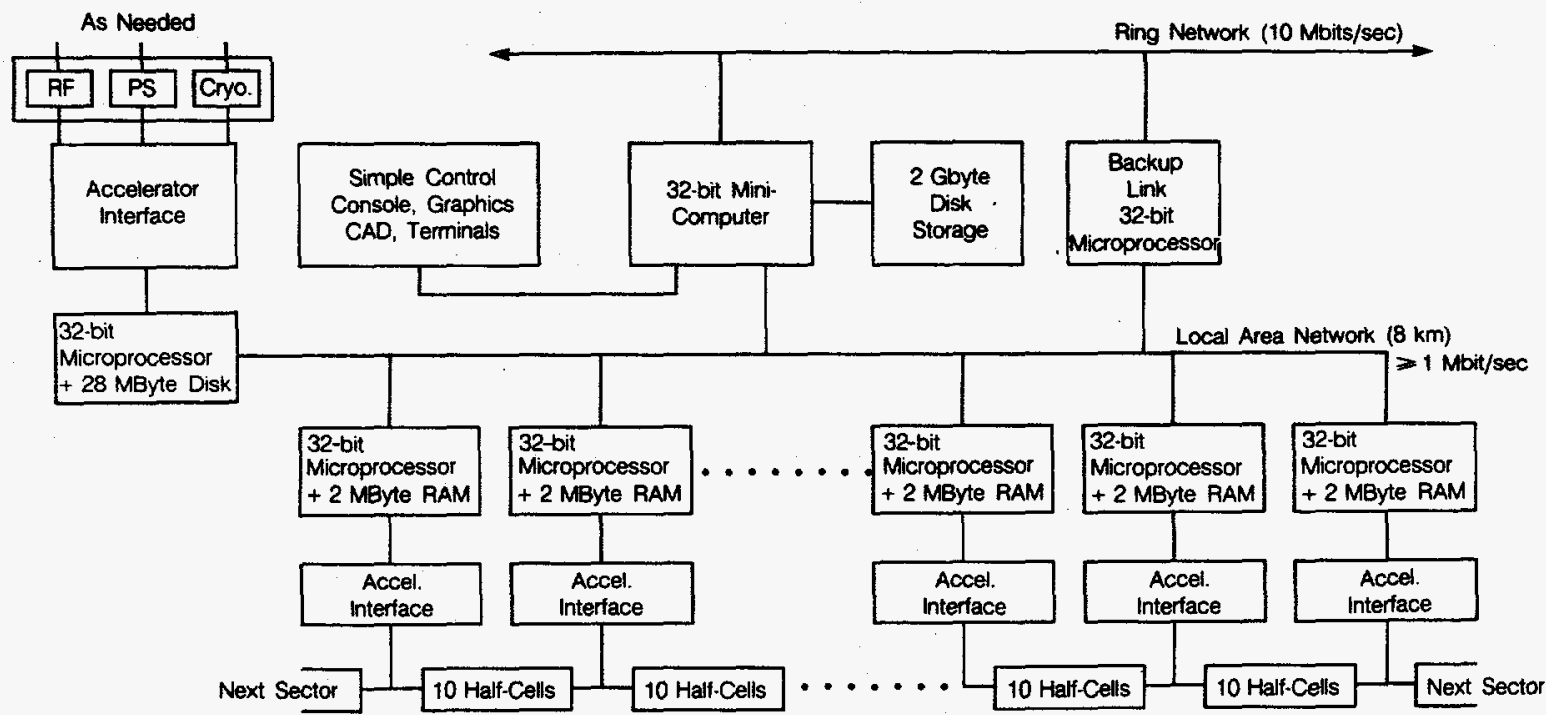

(b)

Figure 5-7. (a) Block diagram of SSC central controls system. (b) Block diagram of sector controls system. 


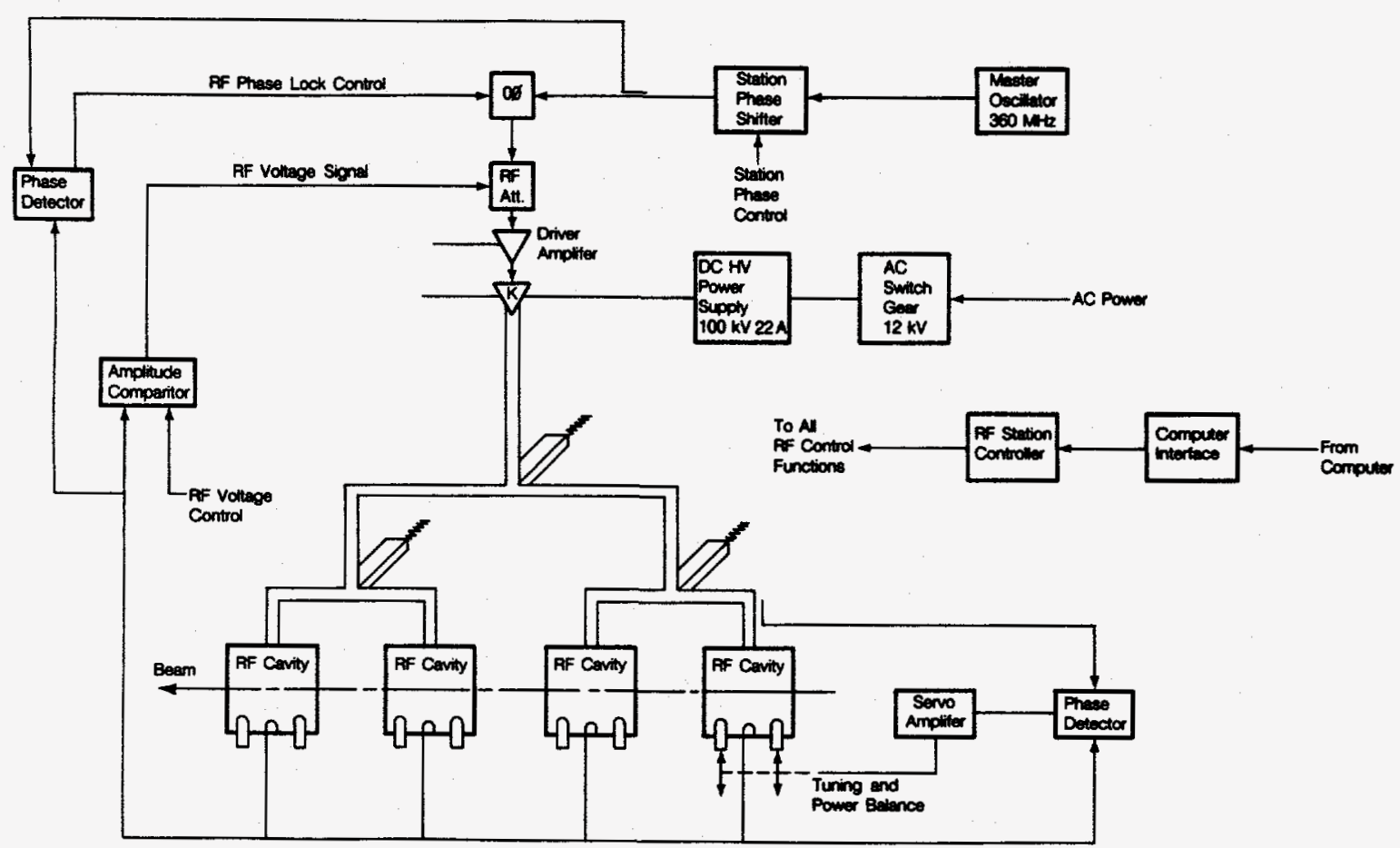

Figure 5-8. Master circuit diagram of rf accelerating system, indicating servo-loops to stabilize the amplitude and phase of the cavity fields. 


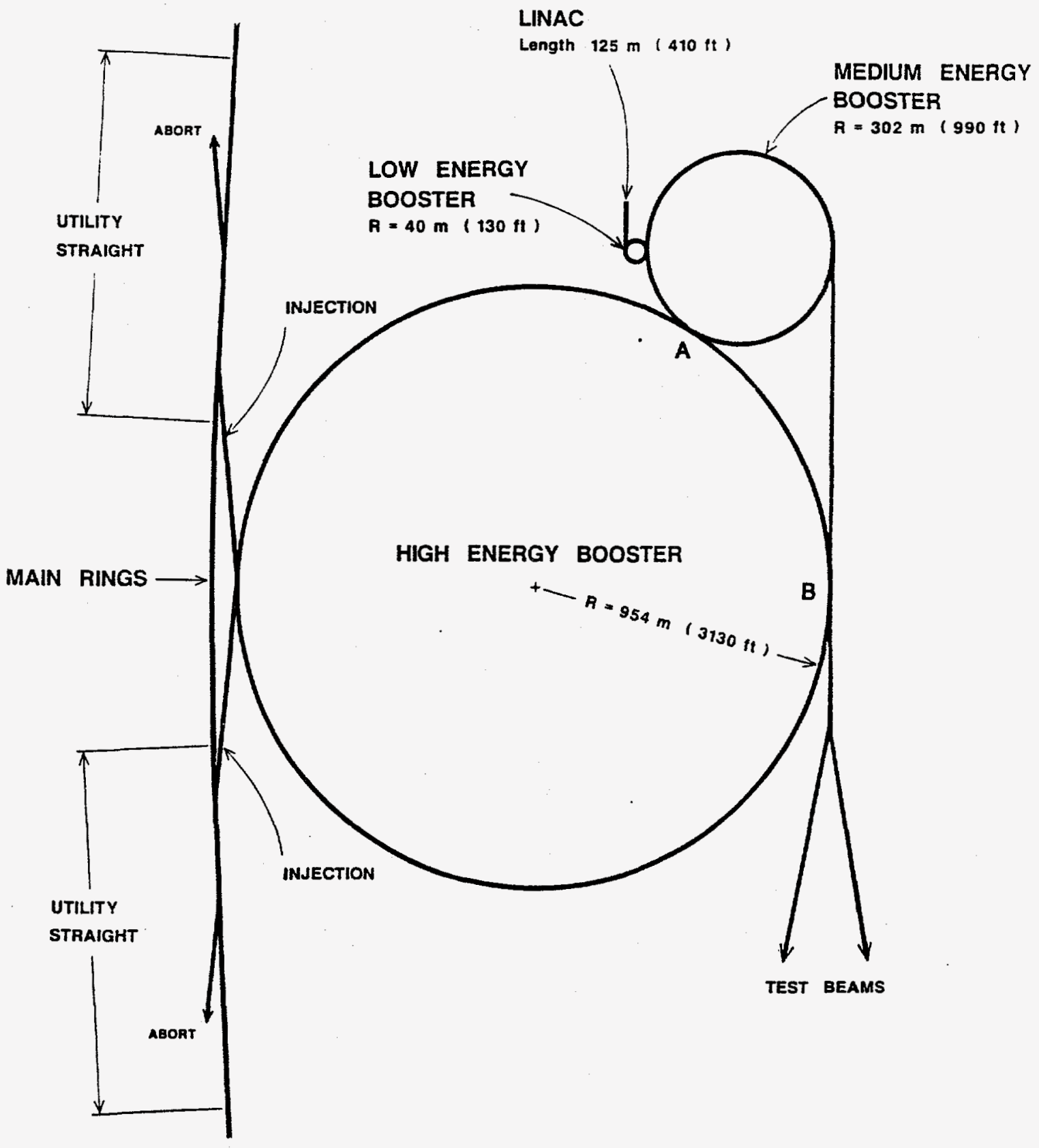

Figure 5-9. Layout of the injector complex. The high energy booster (HEB) can inject protons in both directions into the main ring. The linear accelerator, low energy booster, and medium energy booster can inject protons into the high energy booster at $A$ or $B$, depending on which collider ring is to be filled. 
Table 5-1

SSC Injector Global Parameters

Injected Particie

Injection Momentum

Extraction Momentum

Circumference (length)

RF Frequency

Bunch Spacing

Average Current

Low

Energy

Medium

High

Linac

Booster

Energy

Energy

$\mathrm{H}^{-}$

0.0

$\mathrm{H}^{-}$

1.22

Booster

Booster

Normalized Transverse

Emittance (rms)

0.68

0.75

0.83

0.91

Longitudina 1

Emittance (rms)

0.053

1.6

1.6

37.0

Cycle Time

0.1

0.1

4.0

40.0

mn-mrad

meV-s

$\mathbf{S}$

$\mathrm{GeV} / \mathrm{C}$

$\mathrm{GeV} / \mathrm{c}$

meters

$\mathrm{MHz}$

meters

$\mathbf{m A}$ 
(1) Linear Accelerator

The first link in the injector chain is a linear accelerator beginning with a negative hydrogen ion source followed by three stages of linac, each optimized for the average velocity of the particles being accelerated. The first, a radio frequency quadrupole stage, accelerates to about $2.5 \mathrm{MeV}$, the second, a drift tube linac from 2.5 to about $220 \mathrm{MeV}$ and the third, a side coupled linac accelerating from $220 \mathrm{MeV}$ to $600 \mathrm{MeV}$ kinetic energy. The total length of the linac will be about 125 meters. $\mathrm{H}^{-}$ions are accelerated so that multi-turn, charge exchange injection into the next stage can be used to minimize brightness dilution. Each section of the linac is patterned after devices already in operation, primarily at Los Alamos National Laboratory.

\section{(2) Low Energy Booster and Medium Energy Booster}

These two relatively rapidly cycling synchrotrons are of essentially conventional design, utilizing laminated iron magnets with particular attention paid to smoothness of the beam vacuum envelope to avoid emittance diluting instabilities. Their injection transfer energies are selected to serve the dual purpose of controlling space charge tune shift and avoiding transition energy. At low momentum, the self-repulsion of the beam particles can work together with the focusing properties of the ring to lower the beam brightness. Experience has shown that, by limiting the so-called space charge induced tune shift to $1 / 4$ or less, this effect can be avoided. Similarly, while the additional momentum spread incurred in crossing transition energy would not be harmful, the transient state of high beam density that occurs in transition crossing can lead to brightness dilution through other instabilities, as has been shown by experience. Thus, transition crossing is to be avoided if possible.

In the Low Energy Booster (LEB) the injection (kinetic) energy of $600 \mathrm{MeV}$ (kinetic) holds $\Delta v \simeq 1 / 4$ for the lowest emittances and highest currents expected. Its top energy, $8 \mathrm{GeV}$, is selected low enough that operation always remains below transition energy in this ring. In the Medium Energy Booster (MEB), the beam optical design is selected so that the beam energy is above transition throughout its cycle. It also turns out that the space charge criterion is met in this configuration. LEB and MEB need to be relatively rapidly cycling in order not to add significantly to the cycle time of the High Energy Booster (HEB) which, for economy, must cycle rather slowly. The resulting $10 \mathrm{~Hz}$ repetition rate for the 1 inac and $L E B$ and $1 / 4 \mathrm{~Hz}$ rate for the MEB are well within the state of the art. The top energy of the MEB, $100 \mathrm{GeV}$, is chosen so that transition crossing in the HEB can be avoided.

\section{(3) High Energy Booster}

Injection energy into the collider ring is set by economic considerations. The lower the injection energy, the stronger will be the field distortions due to persistent currents in the superconductor. This effect can be ameliorated by use of very fine filaments in the superconducting cable of the magnets, as discussed in Sect. 3.3. Projections of the development of superconducting cable technology to the time of SSC construction lead us to select 1 TeV as a suitable injection energy for the main ring based on these effects. At $1 \mathrm{TeV}$, economical operation of the HEB requires the use of superconducting technology for that synchrotron. The acceleration ratio of the HEB is about ten, well within demonstrated performance of the Tevatron. 
In essence, the HEB is a miniature version of the main collider, albeit with a lower acceleration ratio making its technology less demanding. The cryogenic needs of the HEB can be met by one of the refrigeration units planned for the main collider. For reliability, however, the HEB cryogenic system will be strongly coupled to that of the collider ring.

\section{(4) Comments}

The entire injector chain is similar in spirit and substance to other cascaded synchrotron complexes. Each component of the chain is similar to an existing device. New is the need to preserve beam brightness scrupulously in each stage. Current understanding based on extensive proton and electron storage ring operation indicates that the beam brightness desiderata can be readily achieved by careful design. 
$\checkmark$ 
103

Chapter 6

PRELIMINARY CONSTRUCTION PLAN

The construction plan presents major tasks and events, and their interrelationships, leading to the completion of the SSC construction. This preliminary construction schedule shows a duration of six years.

\subsection{SUMMARY-LEVEL SCHEDULE}

Figures 6-1 and 6-2 present a summary-level construction schedule for the SSC. The construction phase is assumed to start at the beginning of FY1988 with Congressional approval, authorization, and appropriation of construction funds, and to continue for six years, until the end of FY1993. In order to ensure an efficient and effective construction period, careful and extensive planning during the Phase I R\&D is essential. The milestones keyed to the start of construction in October 1987 assume submission of site proposals by the states starting in early calendar year 1987 followed by site reviews and NAS/NAE evaluations in early FY1988 with an anticipated site selection by September 1988.

At the beginning of the construction period (October 1987) the Phase II Architect-Engineer/ Construction Management (AECM) selection process will begin with the expectation that the $A E / C M$ firm(s) selected will be on board and into early Preliminary Design (Pre-Title I) concurrent with the site studies and the announcement of the site selection. The Title I completion and review and the Title II detail designs for certain critical path items in the construction plan (e.g. South Arc Tunnel sectors for the Collider ring tunnels) would then efficiently proceed, and the actual construction on site would be underway by June 1989. The construction plan shows the first beneficial occupancy for a tunnel sector approximately nine months later (April 1990), and at this point the interrelationships and integration between the first installation. of technical systems (arc sector dipole magnets and "half-cells" of the machine lattice) become apparent.

The "Critical Path" activities focus on the "Collider Ring Dipole Magnet" production and the "Collider Ring Underground Construction"; the scheduling and interrelationships between various sector availabilities in the arc seclions and the cluster hall areas are shown in Fig. 6-2. The collider construction sequence follows a very closely coupled phased construction approach, and as each tunnel sector becomes available for occupancy, the installation and testing of technical components (magnet systems, cryogenics, vacuum systerns, power supplies etc.) follow in a stepwise fashion such that ultimately each sector can begin undergoing acceptance testing as efficiently as possible. As each of these "Collider Ring Sectors" is accepted, the pre-operational testing phase will commence, and the commissioning of each area will proceed sequentially around the ring. 
$\begin{array}{cccccc}\text { CSIR.yr1 } & \text { CSIR.yr2 } & \text { CSIR.yr3 } & \text { CSTR.yr4 } & \text { CSRA. yrs } & \text { CSIR.yr6 }\end{array}$

A. Construcison PLAM

Free

FY89 8490

FY93

\section{PRoJeCi methokizallow}

2 SIIt SELECTEO

3 FIAST CONEENTIOUI COUSTR STARIS AI SIIE

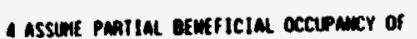
FIAsI IMJecion incils. SECTIOW

5 peosect conplentow

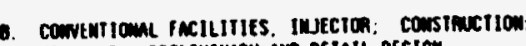

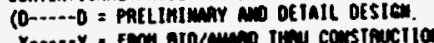

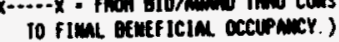

\section{Lumac nom inmersth liues}

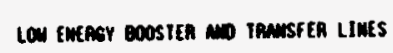

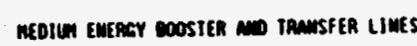
HIEH EMERAY BOOSIEA MO IRMUSFER LIUES IESI BeaM Faciulties

c. Howical srstens, inecion:

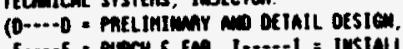

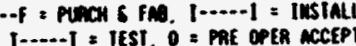

tIme

\section{Low entingr costen}

MEOLUN EMERGY BOOSIER

HIE EMERG BOOSTER

IESt Btams

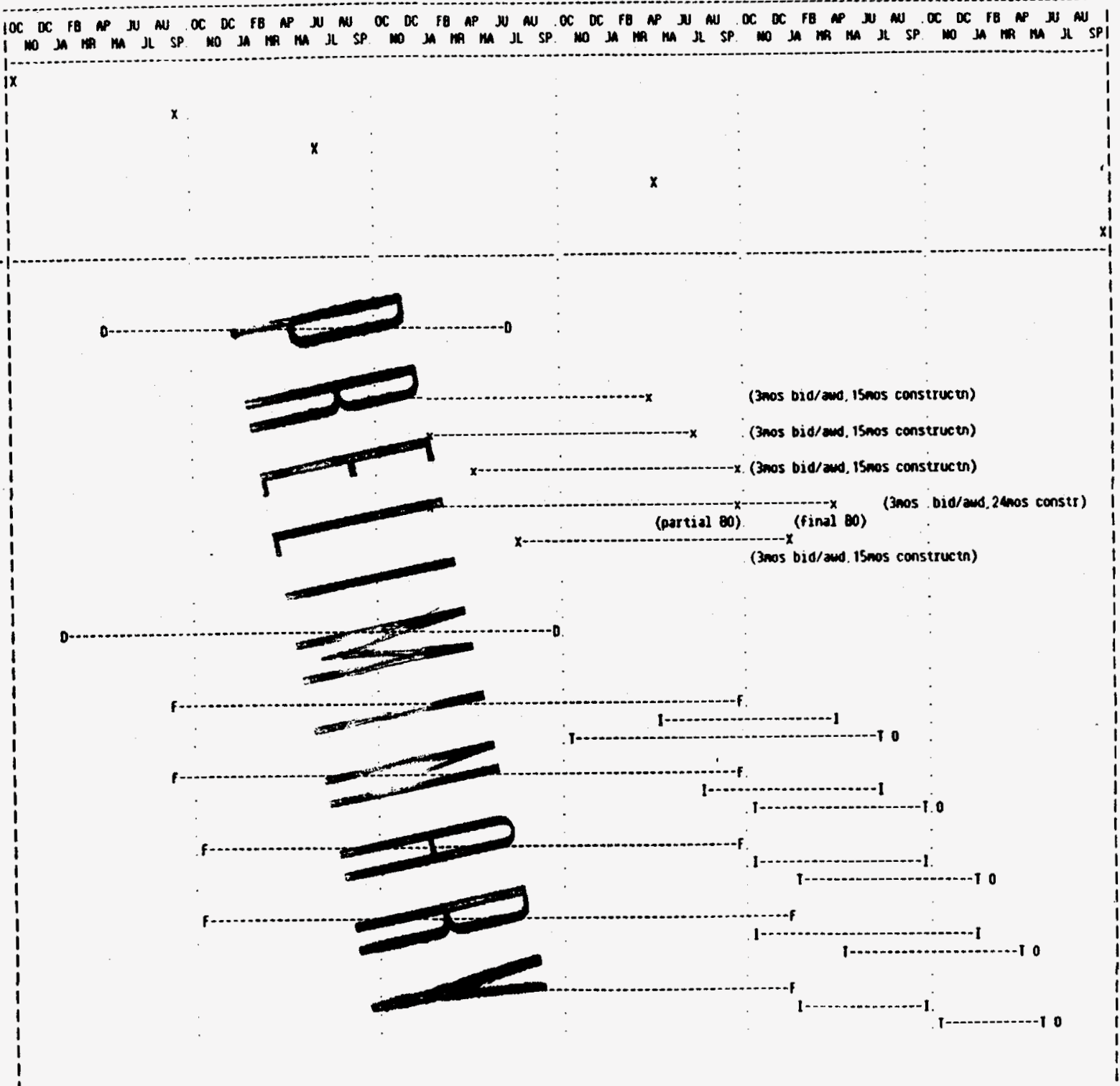

Figure 6-1. Construction schedule, injector summary. 


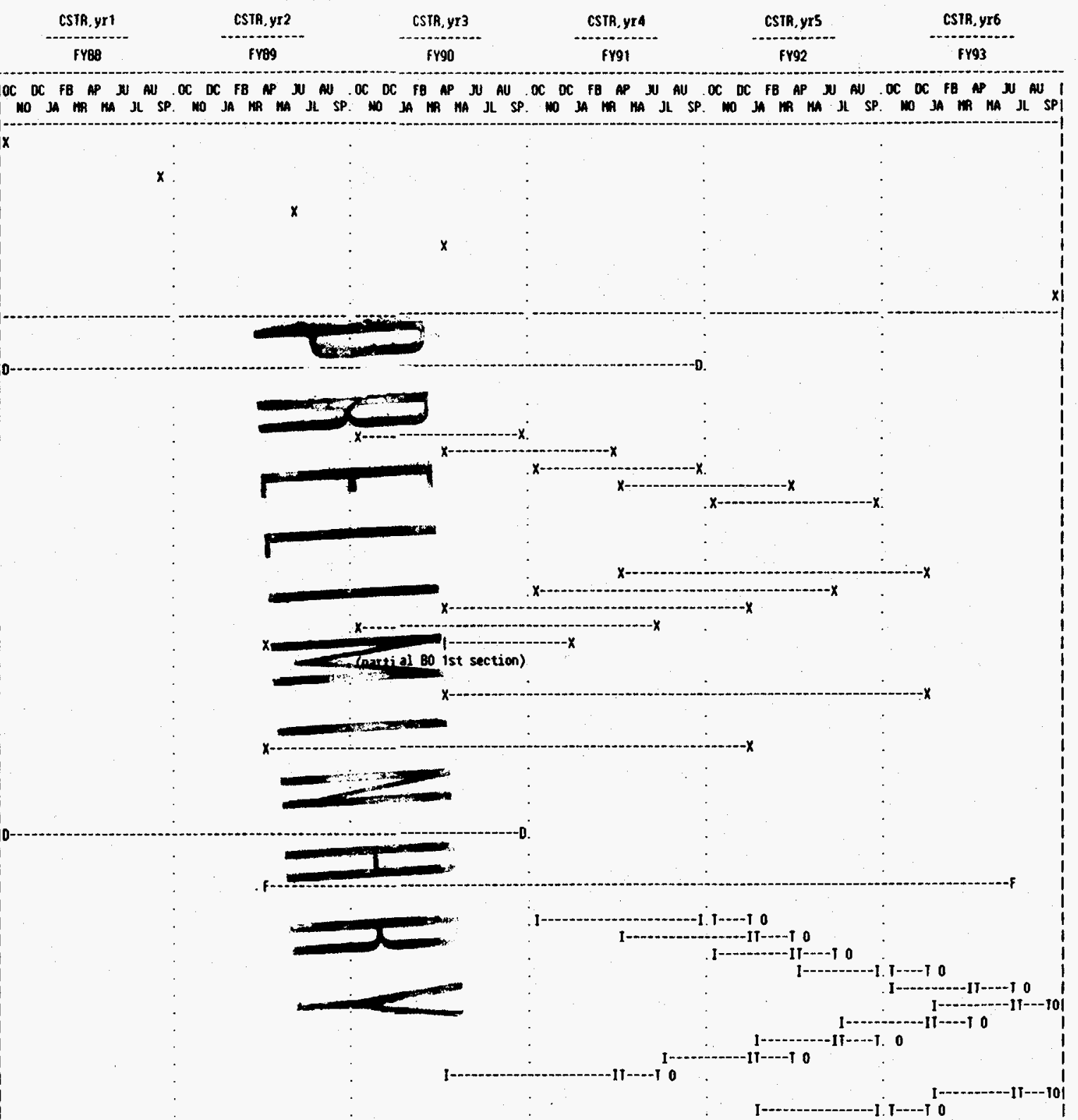

Figure 6-2. Construction schedule, collider ring summary. 
106

\subsection{MAGNET FABRICATION}

During Phase I, development work on the dipole magnets for the collider rings has been emphasized. Dipole magnet models have been assembled at the Laboratories using industrial methods and industrially supplied components. Essential parts of the associated technology have been transferred to industry. These steps will enable the laboratories and industry to plan for a very early and rapid tooling up and start of magnet production early in the construction project. The accomplishments of the Phase I R\&D work will also provide assurance that the SSC organization will be able to release the major contracts for material purchases and magnet assembly contracts that are indicated on the schedule shown on Fig. 6-3. The overall magnet production plan summary described in Fig. 6-3 shows the main dipole magnet fabrication plans along with the quadrupole magnet, spool piece, and other special magnet fabrication and installation plans.

The fabrication and installation of the main collider ring magnets has received considerable attention during the planning studies. The magnet production plan assumes that two (or more) industrial firms will manufacture the nearly 8000 dipole magnets required and ship them to the SSC for final testing and installation. The peak production rate of approximately 600 dipole units per quarter requires an efficient and aggressive work plan. Preliminary manufacturing studies by two industrial firms are already being reviewed, and the phased steps leading to authorized manufacturing contracts by several qualified vendors in early FY7988 are now being planned. Figure 6-3 shows the major dipole fabrication extending from mid-FY1989 (after production tooling is fabricated and installed from April 1988 through March 1989) through approximately May 1993, a period of approximately four years. The fabrication of the quadrupoles and spool pieces starts approximately one year later, in April 1989, and the installation of the half-cell units (each comprised of five dipoles, one quadrupole, and one spool) also commences in mid 1989.

\subsection{INJECTOR SYSTEMS}

Although the initial emphasis in the construction plan centers on the Collider Ring (the overall critical path track), a comprehensive start on the "Injector Systems" is also planned. While the overall start of the injector construction will necessarily await finalization of the detail siting studies and optimizations for the collider ring specification, an early start on the first injector system is planned such that an efficient stepwise "installationtest-acceptance" sequence follows for each of the Linac, Low Energy Booster, Medium Energy Booster, and High Energy Booster accelerators respectively, as indicated in Fig. 6-1.

\subsection{COMMENTS}

The information presented here is a preliminary summary-level schedule of major SSC systems. More complete and lower level details are being developed in the current activities leading to the SSC Conceptual Design Report (April 1986). Final schedule details for both technical and conventional systems will be provided in that document. 


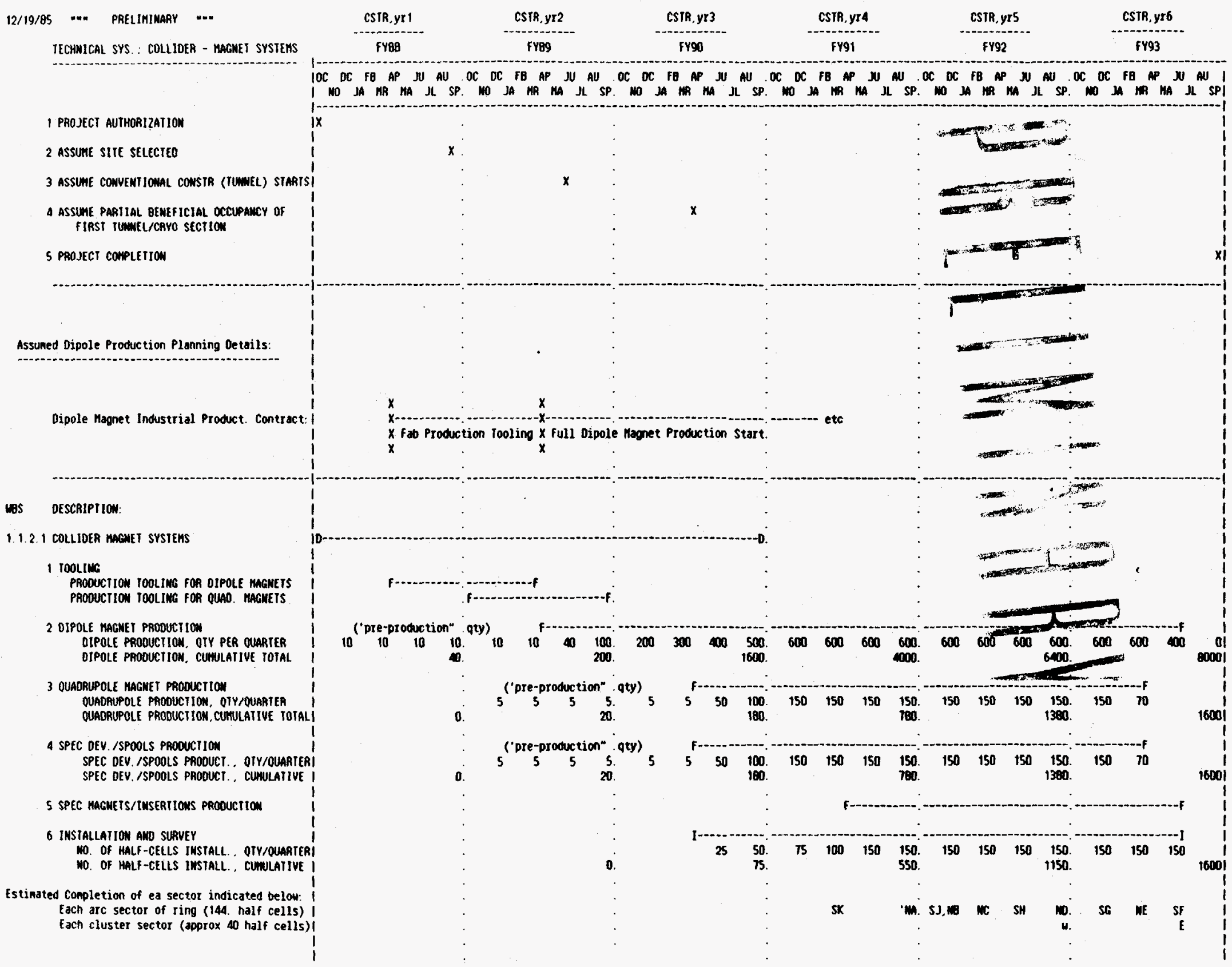

Figure 6-3. Construction schedule, example details for collider magnet systems. 
In concert with the detailed construction schedules, the Conceptual Design Report will provide a comprehensive construction plan including all cost estimates, cost and manpower profiles, milestone charts, and critical path analyses. The manpower projections will identify staffing requirements for management, design, and fabrication efforts during construction. 


\title{
Chapter 7
}

\author{
SUMMARY AND CONCLUSIONS
}

The Superconducting Super Collider or SSC is a proposed high-luminosity proton-proton accelerator and storage ring complex with beams of $20 \mathrm{TeV}$ energy. The magnetic confinement system makes use of the phenomenon of superconductivity to produce high magnetic fields (and so smaller size) and to reduce greatly the electric power needed to operate the facility. Physically the SSC consists mainly of an underground tunnel $83 \mathrm{~km}$ ( $52 \mathrm{miles}$ ) in circumference around which protons circulate in opposite directions within two evacuated beam pipes inside separate rings of magnets. At six points the beams are made to intersect almost head-on. The interpenetrating bunches of protons produce only a few very high energy collisions at each crossing and so can repeatedly collide without degradation. Nevertheless, because oppositely moving bunches pass through each other every hundred millionth of a second, the experimenters studying the reactions have a very large data rate in their detectors. From the myriad of electronic signals recorded from a collision, the experimenters are able to identify the emerging particles and explore the inner dynamics of matter at distances a ten billion billion times smaller than human size.

The experimental discoveries and theoretical developements of the past 20 years have led physicists to a comprehensive description of matter as composed at the elementary level of quarks and leptons interacting via the exchange of force (gauge) particles. In the energy or mass region up to $0.1 \mathrm{TeV}(100 \mathrm{GeV})$, this so-called standard model provides a remarkably successful framework consistent with all known observations. For all its noteworthy triumphs, the standard model still leaves many questions unanswered. It contains a large number (approximately 20) empirical parameters a reflection of its failure to explain the origin and pattern of particle masses, the observed groupings of quarks and leptons, the reason for the handedness of weak interactions, to name a few.

To address these and other unanswered questions, more powerful instruments are needed. A decade ago plans were laid to move beyond present capabilities. The Tevatron collider at Fermilab, the SLC at Stanford University, and LEP at the European Laboratory for Particle Physics (CERN) will soon begin to explore the edges of the new energy domain. But there are compelling reasons why, even as these facilities commence operation, a new instrument is required. The electron-positron colliders (SIC., IEP) will study the $Z$ mass region of $0.1 \mathrm{TeV}$ in detail. The Tevatron, with its colliding $1 \mathrm{TeV}$ protons and antiprotons, will reach $0.3 \mathrm{TeV}$.

The standard model has so many correct aspects that one can tell with considerable certainty at what moss scale its obscurities will surely be illuminated. That mass is $1 \mathrm{TeV}$ or greater. Only a high-luminosity multi-TeV hadron collider such as the SSC has the capacity to explore this mass range. The spectrum of predicted new particles and forces can be used to delimit the required parameters. Beam energies of $20 \mathrm{TeV}$ and a luminosity of $10^{33}$ $\mathrm{cm}^{-2} \mathrm{~s}^{-1}$ are pinpointed as appropriate for the SSC. 
Despite its unprecedented size, the SSC is a natural and modest extension of existing accelerator technology and practice. Its technical basis lies in the cascaded synchrotrons of the world's major high energy physics laboratories, of which Fermilab in the U.S., CERN in Europe, and Serpukhov in the U.S.S.R. are examples. Fermilab is particularly relevant because of its pioneering use of superconducting accelerator magnets in the Tevatron.

Four years ago it was appreciated that the SSC was technologically feasible, with the Tevatron pointing the way. Two years ago, after a number of workshops and studies and a recommendation from the High Energy Physics Advisory Pane 1, the U.S. Department of Energy (DOE) initiated research and development activities to sharpen the design criteria and cost estimate of the SSC, as well as address identified technical problems. The R\&D effort put major emphasis on superconducting magnets, the costliest single technical component of the system. An extensive model magnet program at Brookhaven National Laboratory, Fermilab, Lawrence Berkeley Laboratory and the Texas Accelerator Center has been remarkably successful. Enough high quality models have been made to establish one magnet style as superior and to assure that reproducible magnets of excellent field quality, field strength, and reliability can be built with methods that are applicable to large-scale industrial production.

Other aspects of the R\&D program concerning the cost have been the development of improved superconducting wire in cooperation with industry, a study of beam dynamics to establish the smallest prudent transverse size of the magnets, and experiments on cold evacuated beam pipes in the presence of synchrotron radiation to assess the pumping needs for the SSC vacuum.

The two-year R\&D effort has shown that the components of the SSC are thoroughly understood and that a reliable cost estimate and sensible construction plan for the complete facility can be developed. The Conceptual Design Report, of which this is a preview, will provide that information.

The general features of the SSC, both physically and technically, are described in Chapters 4 and 5 . Briefly, the facility is mostly underground. Its $83 \mathrm{~km}$ tunnel is to be accessed only occasionally along its length. The oval shape of the ring is $28 \mathrm{~km}$ (17 miles) by $23 \mathrm{~km}$ (14 miles). On opposite sides are two clusters of interaction regions and other facilities. Apart from these areas and in a 200 meter wide path above the tunne 1 , the land encompassed by the SSC is undisturbed and available for normal use. The clusters have complexes of experimental support buildings, the cascade of smaller accelerators of the injector, and the central laboratory and office area.

The largest number of the technical components are distributed in the tunne1. There are two complete beam pipes and magnetic confinement systems, consisting of regular arrays of bending and focusing magnets, nearly 10,000 in a11, interrupted eight times around the tunnel by special sets of magnets for the interaction regions.or the injection and abort sections. There are ten main refrigeration stations around the ring to provide the liquid helium and nitrogen to cool the magnets to their superconducting state. Elaborate distributed controls systems are provided to maintain the beams in proper paths through the evacuated beam pipes for a day or more before refilling. 
The protons stored in the main rings of the SSC are produced by the injector, a cascade of synchrotrons the size and complexity of a major facility like the Tevatron. A linear accelerator followed by three increasingly large synchrotrons boost the energies of the protons to $1 \mathrm{TeV}$ before injection into the main rings, where they are then accelerated to the final energy of $20 \mathrm{TeV}$. All of the sub-systems of the booster synchrotrons and of the main collider ring are similar in kind to components of existing machines. The only difference of the SSC is the absolute numbers.

Planning work on a construction schedule for the Conceptual Design Report is exploring the possibility of a six year construction period, with successive parts of the complex being occupied as they become available. (See Chapter 6.) Installation and testing of the technical systems would start typically in the third year. Assuming an optimal spending profile and congressional authorization substantially before site selection, the critical path is dominated by site selection. Because technical component manufacture will be carried out by industries worldwide, with established facilities, component manufacture can begin soon after notice to proceed. Completion of the facility will then be paced by the availability of conventional parts of the facility for installation and testing of technical systems.

Given a robust and aggressive DOE construction program, the SSC could be on line for physics research in 1994. 